\title{
Automated Coverage Calculation and Test Case Generation
}

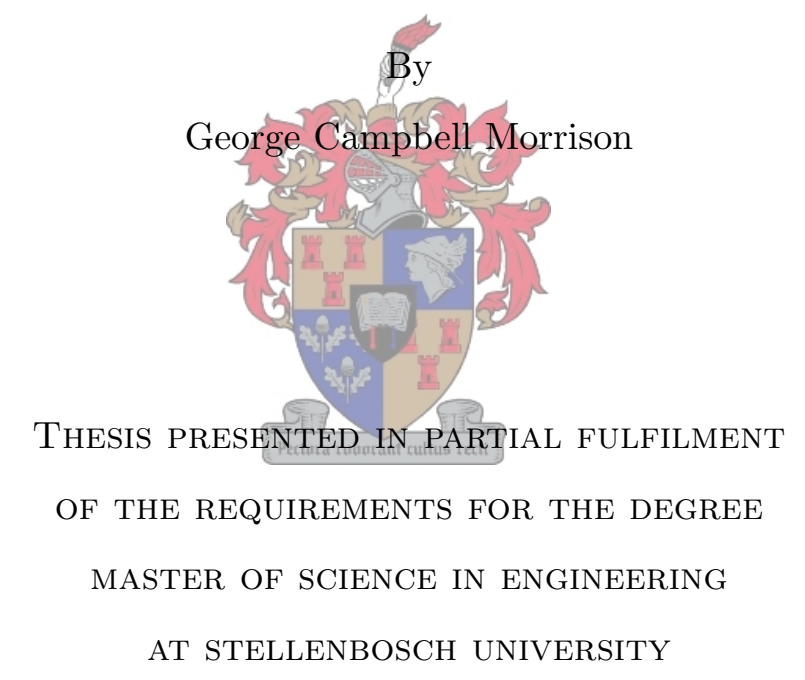

Supervisors: Dr C. P. Inggs

Department of Mathematical Sciences: Computer Science

Mr A. Barnard

Department of Electrical \& Electronic Engineering

March 2012 


\section{Declaration}

By submitting this thesis electronically, I declare that the entirety of the work contained therein is my own, original work, that I am the sole author thereof (save to the extent explicitly otherwise stated), that reproduction and publication thereof by Stellenbosch University will not infringe any third party rights and that I have not previously in its entirety or in part submitted it for obtaining any qualification.

March 2012

Copyright (C) 2012 Stellenbosch University

All rights reserved 


\section{Abstract}

This research combines symbolic execution, a formal method of static analysis, with various test adequacy criteria, to explore the effectiveness of using symbolic execution for calculating code coverage on a program's existing JUnit test suites. Code coverage is measured with a number of test adequacy criteria, including statement coverage, branch coverage, condition coverage, method coverage, class coverage, and loop coverage. The results of the code coverage calculation is then used to automatically generate JUnit test cases for areas of a program that are not sufficiently covered. The level of redundancy of each test case is also calculated during coverage calculation, thereby identifying fully redundant, and partially redundant, test cases. The combination of symbolic execution and code coverage calculation is extended to perform coverage calculation during a manual execution of a program, allowing testers to measure the effectiveness of manual testing.

This is implemented as an Eclipse plug-in, named $A T C O$, which attempts to take advantage of the Eclipse workspace and extensible user interface environment to improve usability of the tool by minimizing the user interaction required to use the tool.

The code coverage calculation process uses constraint solving to determine method parameter values to reach specific areas in the program. Constraint solving is an expensive computation, so the tool was parallellised using Java's Concurrency package, to reduce the overall execution time of the tool. 


\section{Opsomming}

Hierdie navorsing kombineer simboliese uitvoering, 'n formele metode van statiese analise, met verskeie toets genoegsaamheid kriteria, om die effektiwiteit van die gebruik van simboliese uitvoer te ondersoek vir die berekening van kode dekking op 'n program se bestaande JUnit toets stelle. Kode dekking word gemeet deur verskeie toets genoegsaamheid kriteria, insluited stelling dekking, tak dekking, kondisie dekking, metode dekking, klas dekking, en lus dekking. Die resultate van die kode dekking berekeninge word dan gebruik om outomaties JUnit toets voorbeelde te genereer vir areas van 'n program wat nie doeltreffend ondersoek word nie. Die vlak van oortolligheid van elke toets voorbeeld word ook bereken gedurende die dekkingsberekening, en daardeur word volledig oortollige, en gedeeltelik oortollige, toets voorbeelde identifiseer. Die kombinasie van simboliese uitvoer en kode dekking berekening is uitgebrei deur die uitvoer van dekking berekeninge van 'n gebruiker-beheerde uitvoer, om sodoende kode dekking van 'n gebruiker-beheerde uitvoer van 'n program te meet. Dit laat toetsers toe om die effektiwiteit van hulle beheerde uitvoer te meet.

Bogenoemde word geïmplimenteer as 'n Eclipse aanvoegsel, genaamd ATCO, wat poog om voordeel te trek vanuit die Eclipse werkspasie, en die uitbreibare gebruiker oordrag omgewing, om die bruikbaarheid van $A T C O$ te verbeter, deur die vermindering van die gebruiker interaksie wat benodig word om ATCO te gebruik.

Die kode dekking berekeningsproses gebruik beperking oplossing om metode invoer waardes te bereken, om spesifieke areas in die program te bereik. Beperking oplossing is 'n duur berekening, so ATCO is geparalleliseer, met behulp van Java se Concurrency pakket, om die algehele uitvoer tyd van die program te verminder. 


\section{Acknowledgements}

I would like to thank my supervisor, Dr Cornelia Inggs, who has gone out of her way to help me complete this thesis. Her guidance, expertise, and willingness to help, even at uncomfortable times, has truly been invaluable to me during this time. I would also like to thank Mr Arno Barnard, my co-supervisor at the Faculty of Engineering, whose involvement allowed me to do the M.Sc.Eng degree.

I would also like to express my sincerest gratitude to my girlfriend, family, and friends, who have been very understanding, motivating, and supportive, while I was working on this thesis. Working full-time, while doing this thesis part-time, has been an incredible challenge for me, and I would not have succeeded without their support.

Lastly, I would like to thank my superiors at S1 Corporation, who have been very accommodating to my studies, while I was trying to finish this thesis after hours. 


\section{Contents}

1 Introduction $\quad 1$

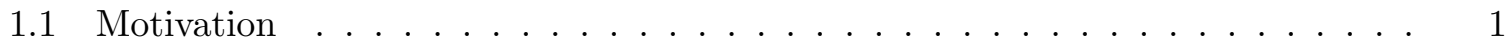

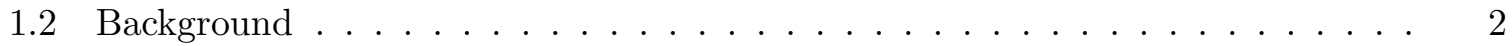

1.2.1 Symbolic Execution . . . . . . . . . . . . . . . . . 2

1.2 .2 Code Coverage . . . . . . . . . . . . . . 4

1.2.3 JUnit Test Generation . . . . . . . . . . . . . . . . . 6

1.3 Literature Synopsis . . . . . . . . . . . . . . . . . . . . . 6

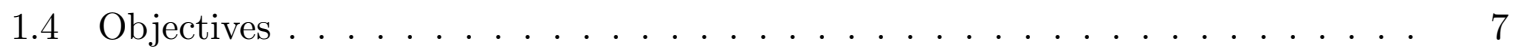

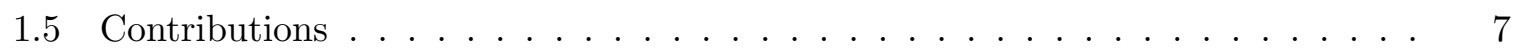

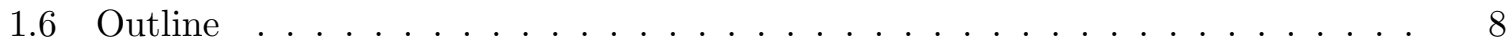

2 Theoretical Background $\quad 11$

2.1 Introduction to Program Verification and Program Analysis . . . . . . . . . 11

2.2 Program Verification . . . . . . . . . . . . . . . . . . . 12

2.2 .1 Model Checking . . . . . . . . . . . . . . . . . . 12

2.2 .2 Theorem Proving . . . . . . . . . . . . . . . . . . . . 13

2.2.3 Program Verification Tools to Prove the Absence of Bugs . . . . . . . . 14

2.2.4 Program Verification Tools to Find Bugs . . . . . . . . . . . . . . . . 14

2.2 .5 Conclusion . . . . . . . . . . . . . . . . . . 14

2.3 Program Analysis . . . . . . . . . . . . . . . . . . . . 15

2.3.1 Program Analysis Tools to Prove the Absence of Bugs . . . . . . . . . . 17

2.3.2 Program Analysis Tools to Find Bugs . . . . . . . . . . . . . . 17

2.3 .3 Conclusion . . . . . . . . . . . . . . . . . . 17 


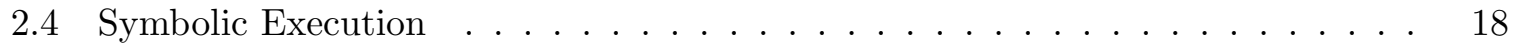

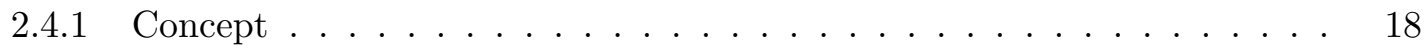

2.4 .2 Path Condition . . . . . . . . . . . . . . . . . . 20

2.4.3 Symbolic Execution Tree . . . . . . . . . . . . . . . . . . 21

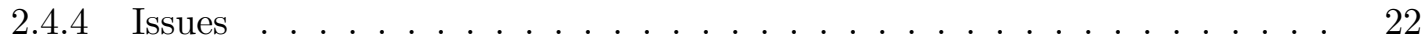

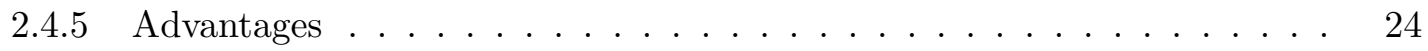

2.4 .6 Extending Symbolic Execution $\ldots \ldots \ldots \ldots \ldots$

2.4.7 Symbolic Execution in Practice . . . . . . . . . . . . . . 27

2.5 Dynamic Analysis . . . . . . . . . . . . . . . . . . . . 28

2.5.1 Focused Testing using Program Analysis . . . . . . . . . . . . . . 29

2.6 Introduction to Test Adequacy and Coverage $\ldots \ldots \ldots \ldots$

2.7 Test Adequacy Criteria . . . . . . . . . . . . . . . . . . . . 31

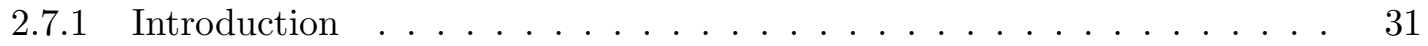

2.7.2 Categories of Test Data Adequacy Criteria . . . . . . . . . . . 32

2.7 .3 Focus of this Thesis $\ldots \ldots \ldots \ldots \ldots \ldots \ldots$

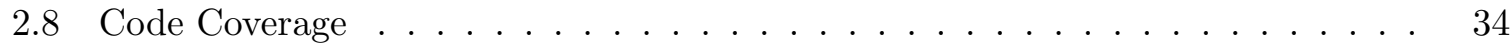

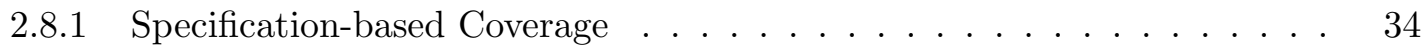

2.8 .2 Program-based Coverage $\ldots \ldots \ldots \ldots \ldots \ldots$

2.8 .3 Measuring Coverage . . . . . . . . . . . . . . . . . . . 39

2.8 .4 Coverage Tools . . . . . . . . . . . . . . . . . . . 39

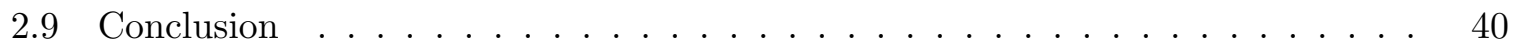

3 Design and Implementation $\quad 41$

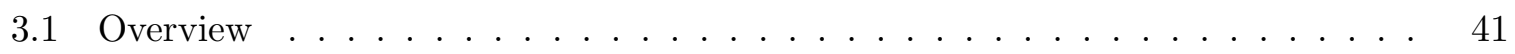

3.2 Information Gathering Phase $\ldots \ldots \ldots \ldots \ldots \ldots$

3.2.1 Artemis' Symbolic Execution Engine . . . . . . . . . . . . . . . . 42

$3.2 .2 \quad$ Symbolic Execution Tree $\ldots \ldots \ldots \ldots$. . . . . . . . . . 45

3.3 Information Analysis Phase $\ldots \ldots \ldots \ldots \ldots \ldots \ldots$

3.3 .1 Execution Tracing $\ldots \ldots \ldots \ldots \ldots \ldots \ldots \ldots \ldots$

3.3 .2 Test Environment $\ldots \ldots \ldots \ldots \ldots \ldots \ldots \ldots \ldots$

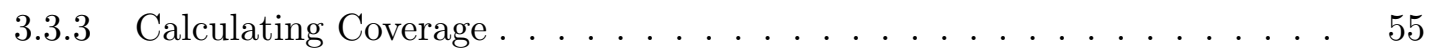

3.3 .4 Test Redundancy Detection . . . . . . . . . . . . . . . . . . 63 


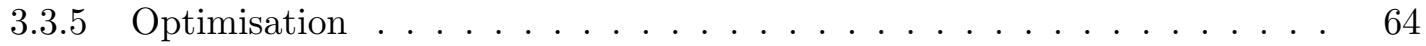

3.3.6 Execution Tracing of Manual Testing . . . . . . . . . . . . . . . 65

3.4 Result Analysis Phase . . . . . . . . . . . . . . . . . 65

3.4 .1 Artemis' Test Generation Engine . . . . . . . . . . . . . . . . 66

3.4 .2 Test Generation in $A T C O \ldots \ldots \ldots \ldots$

3.5 Eclipse Plug-in . . . . . . . . . . . . . . . . . . 66

3.5.1 Resource Management Plug-in . . . . . . . . . . . . . . 67

3.5.2 Workbench Plug-in . . . . . . . . . . . . . . . . . 67

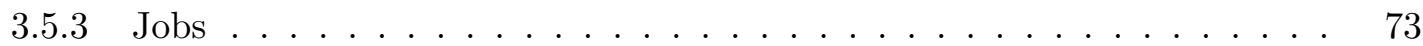

4 Evaluation $\quad \mathbf{7 5}$

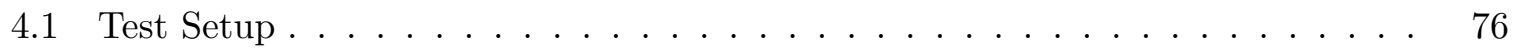

4.1 .1 Single-core GUI Environment . . . . . . . . . . . . 76

4.1.2 Multi-core Command-Line Environment . . . . . . . . . . . . . . 76

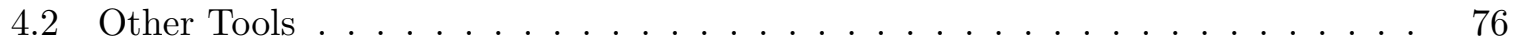

4.3 Correctness of Coverage Calculation . . . . . . . . . . . . . . 77

4.3 .1 Custom Applications . . . . . . . . . . . . . . 77

4.3.2 Small Real-World Applications . . . . . . . . . . . . . . . . . . 81

4.3.3 Large Real-World Application . . . . . . . . . . . . . . . . . . . . 84

4.4 Performance of Coverage Calculation . . . . . . . . . . . . . 86

4.4 Custom Applications . . . . . . . . . . . . . . 87

4.4 .2 Large Real-World Applications . . . . . . . . . . . . . . . . . . 88

4.5 Effectiveness of Generated Test Cases . . . . . . . . . . . . . . . . . . . 89

$\begin{array}{lll}5 & \text { Conclusion } & 91\end{array}$

5.1 Future Work . . . . . . . . . . . . . . . . . . . . . 93

$\begin{array}{ll}\text { Bibliography } & 95\end{array}$ 


\section{List of Tables}

4.1 ATCO Correctness Evaluation: Custom Applications . . . . . . . . . . . . . 79

4.2 ATCO Correctness Evaluation: Custom Applications with EMMA . . . . . . 80

4.3 ATCO Correctness Evaluation: Small Applications . . . . . . . . . . . . . 82

4.4 ATCO Correctness Evaluation: Small Applications with EMMA . . . . . . . 83

4.5 ATCO Correctness Evaluation: Large Application . . . . . . . . . . . . 85

4.6 ATCO Correctness Evaluation: Large Application with EMMA . . . . . . . 86

4.7 ATCO Performance Evaluation: Custom Application . . . . . . . . . . . . . 87

4.8 ATCO Performance Evaluation: Large Application . . . . . . . . . . . . . 88

4.9 ATCO Effectiveness of Generated Test Cases Evaluation . . . . . . . . . . . . 90 


\section{List of Figures}

2.1 A symbolic representation of expressions . . . . . . . . . . . . . . . . . . 19

2.2 A code and graph example of path conditions . . . . . . . . . . . . . 21

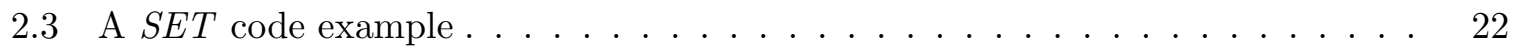

2.4 A $S E T$ graph . . . . . . . . . . . . . . . . . . . . . 23

2.5 A code example to illustrate symbolic loops . . . . . . . . . . . . . . . . . . 24

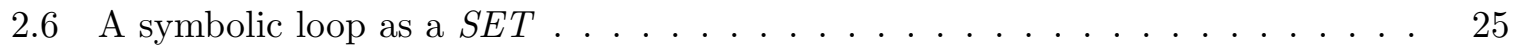

2.7 A code example of infeasible statements . . . . . . . . . . . . . . . 36

2.8 Line coverage . . . . . . . . . . . . . . . . . 36

3.1 An interprocedural infeasible path identification example . . . . . . . . . . . 45

3.2 A Java switch statement example . . . . . . . . . . . . . . . . . . 46

3.3 A SET of a Java switch statement . . . . . . . . . . . . . . . . . . 47

3.4 A simple code example to illustrate interprocedural analysis . . . . . . . . . . 48

3.5 A SET that illustrates branching during interprocedural analysis . . . . . . . 49

3.6 A SET of a symbolic loop with grouped statements _ . . . . . . . . . . 50

3.7 Breakpoint configuration . . . . . . . . . . . . . . 56

3.8 Breakpoint configuration $S E T \ldots \ldots \ldots \ldots \ldots \ldots \ldots \ldots$

3.9 Breakpoint configuration with multiple states $\ldots \ldots \ldots \ldots$

3.10 Breakpoint configuration $S E T$ with multiple states . . . . . . . . . . . 60

3.11 A $S E T$ with coverage counters . . . . . . . . . . . . . . 61

3.12 Interesting case with symbolic loops $\ldots \ldots \ldots$. . . . . . . . . . . 62

3.13 Main preferences page . . . . . . . . . . . . . . . 68

3.14 Symbolic execution preferences page . . . . . . . . . . . . . . . . . 69

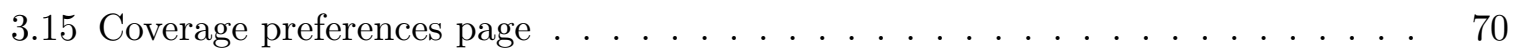


3.16 Manual execution preferences page . . . . . . . . . . . . . 70

3.17 The standard Window drop down menu in Eclipse houses the preferences page. 70

3.18 File coverage view . . . . . . . . . . . . . . . . . 71

3.19 Detailed file coverage view . . . . . . . . . . . . . . . . . . 72

3.20 JUnit statistics view . . . . . . . . . . . . . . . . . . . . 72

3.21 Detailed JUnit statistics view . . . . . . . . . . . . . . . . . . . . . 72

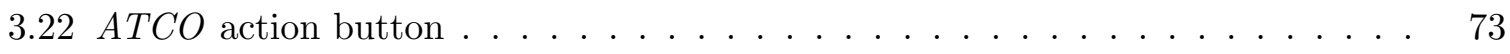

3.23 Progress par. . . . . . . . . . . . . . . . . . . . 74 


\section{Chapter 1}

\section{Introduction}

\subsection{Motivation}

Software testing is a vital part of software development. However, it is a laborious task that typically accounts for half of the software development cycle [45]. This has encouraged the development of various test automation frameworks and tools, to alleviate the effort of performing software testing. One such framework is the JUnit testing framework [29].

The JUnit testing framework allows software developers to create test cases, which consist of class instantiations, and method invocations, with specific parameters, which can be executed by the framework to automatically test the program. A set of these test cases is called a test suite, and a test suite that aims to test the current functionality of a program is called a regression test suite. Having a thorough and efficient regression test suite assists in detecting newly introduced program faults quickly. However, to create and maintain such a regression test suite is an intensive, time-consuming task.

A number of tools attempt to address this problem by providing mechanisms to automatically generate test cases. These mechanisms range from generating random inputs for methods (JCrasher [13]), to using symbolic execution [33] to create a symbolic representation of a program's execution (Symstra [45]), to many more. However, simply generating (often large sets of) test cases is not sufficient to acquire a thorough and efficient regression test suite.

A regression test suite is considered to be thorough if it executes an adequate percentage of the entire program. For example, a test suite may be considered adequate if $80 \%$ of all statements are covered. However, a thorough regression test suite does not necessarily imply 
that it is an efficient test suite.

An efficient regression test suite achieves adequate levels of coverage with the minimum number of test cases. The more test cases contained in a test suite, the longer it will typically take to execute the suite. Regression test suites may often contain redundant test cases, i.e., test cases that do not test any area of the program that has not previously been covered by another test case in the suite. These redundant test cases increase the time, and resources, required to execute a regression test suite, without necessarily contributing to the effectiveness of the suite.

There are tools such as Agitar [1, 45, 46], JTest [27, 45, 46], and Symstra [45], that aim to generate thorough regression test suites. This is achieved by analysing the program, typically through a static analysis methodology $[27,45]$, and then generating test cases to cover as much of the program as possible. Tools, like Symstra, apply redundancy detection checks to ensure that as few redundant tests are created as possible. However, these tools create a separate regression test suite, not considering the content of an already existing regression test suite.

The purpose of this thesis is to explore the practical applicability of using the static analysis formal method called symbolic execution, to extend the current regression test suite by generating additional test cases, and adding them to the suite. The aim is for the resulting regression test suite to be as thorough and efficient as possible.

\subsection{Background}

This section will provide an overview of what is meant by symbolic execution [33], code coverage [47], and automated JUnit test case generation.

\subsubsection{Symbolic Execution}

The notion of symbolic execution follows naturally from normal execution. The code is inspected line-by-line, much like an interpreter would execute machine code. However, instead of actually executing the code, the execution of the program is simulated by inspecting the code and maintaining a representation of the static state of the program. This representation of the static state is called the symbolic state. The symbolic execution of a program must be structured in such a way that the symbolic state is equivalent to the static state of the program, 
during normal execution.

When a procedure is symbolically executed, execution proceeds normally until any data, external to the procedure, is accessed. Data external to the procedure are data entities, like parameter variables of the procedure, global variables in the program, Input/Output operations, and others. Whenever an external data entity is accessed, that entity is regarded as a symbolic value. These symbolic values are used to represent some unknown, yet fixed value. Values that are not external to the procedure are known as concrete values, and include local variables with non-symbolic values assigned to them, or constants.

There are two situations where the symbolic state will manipulate data differently from normal execution. These two situations are evident during the computation of expressions and during conditional branching.

\section{Computation of Expressions}

Since the symbolic values are unknown, any expressions containing symbolic values cannot be solved. Instead, these expressions are represented in terms of the symbolic values within them.

\section{Conditional Branching}

Conditional branching in a program occurs at every branching statement, such as an if statement, where only one of the paths is followed given the conditions in the if. During symbolic execution, concrete conditions, i.e., conditions composed of only concrete values, are handled normally, since the result of the condition can be calculated and the correct execution path can be followed.

Since symbolic values are unknown, if statements with one or more symbolic conditions, cannot be resolved during symbolic execution. Therefore, these conditional statements are referred to as unresolved conditional statements. Whenever an unresolved conditional statement is encountered, it has to be assumed that the condition can be both true and false. This occurs, because it has to be assumed that a symbolic value can represent any value within the domain of its type, i.e., if the symbolic value $S V$ is of type int, it can assume any value in the domain $-2^{31}<=S V<=2^{31}-1$. Since the condition has to be assumed as both true and false, both execution paths need to be followed. When both paths are followed, it means that the execution forks into two parallel executions of the current procedure, where the execution 
of each path proceeds as normal, except for the assumed outcome of the unresolved conditional statement.

The assumptions are stored with their values in the symbolic state as a path condition, because assumptions made by one unresolved conditional statement may assist in resolving subsequent unresolved statement executions.

\section{Path Condition}

The path condition $(P C)$ is an accumulator of conditions on symbolic values which determines a unique control path through the program. Each path has its own $P C$ and no two $P C$ s are ever identical.

At the beginning of a program, the $P C$ is set to true. When a conditional branching statement is encountered, its condition (represented by $b(C)$ ) is examined. If $b(C)$ contains no symbolic values, i.e., only concrete values, then $b(C)$ can be solved using the concrete values. The correct path can then be followed with no modification to the $P C$. However, if $b(C)$ is an unresolved condition, the execution has to be forked into two parallel executions. The two forked executions will each receive a copy of the $P C$, at that point, and the assumed result of $b(C)$ is added to the $P C$ :

$$
\begin{array}{ll}
P C_{1} & \wedge \quad b(C) \\
P C_{2} & \wedge \quad \neg b(C)
\end{array}
$$

Execution of each path continues in parallel, where the path represented by $P C_{1}$ assumes $b(C)=$ true and the path represented by $P C_{2}$ assumes $\neg b(C)=$ true.

\subsubsection{Code Coverage}

Code coverage, a field of study under test adequacy [47], is the measurement of a coverage criterion. A coverage criterion is a testing requirement, which specifies that some element of a program should be covered. To cover an element of a program means to execute or analyse that element during testing. The coverage criteria considered in this thesis may be categorised as control-flow criteria.

Control-flow criteria is specified over the coverage of the control-flow graph of a program. The control-flow graph is a graphic interpretation of the execution of a program, where the nodes represent code blocks, branches occur at conditional statements, and the edges represent 
the conditions required to hold for that path to be followed. Every execution of a program corresponds to a path in the control-flow graph, known as the execution path, from the begin node to the end node. The begin node represents the entry point of the program, and the end node represents the point of program termination.

The four common coverage criteria specified over the control-flow graph are:

- Statement coverage: Statement coverage is a very basic testing requirement which specifies that every statement in the code should be executed at least once.

- Branch coverage: Branch coverage specifies that each possible branch of every branching statement should be executed at least once.

- Method coverage: Method coverage is the testing requirement that every method in a program should be executed at least once.

- Class coverage: This testing requirement specifies that every class in a program should be executed at least once, i.e., any one of its methods should be executed at least once.

An additional, uncommon, coverage criterion considered in this thesis is loop coverage. This testing requirement specifies that every loop in a program should be iterated at least twice. The purpose of loop coverage is to detect bugs that present themselves when a loop is iterated more than once [30]. By this definition, loops, designed to iterate only once or never at all, will never satisfy the loop coverage criterion.

\section{Measuring Code Coverage}

There are various approaches to measure the criteria. Atlassian, the creators of Clover [9, 46], identified three approaches to measure code coverage of Java programs:

1. Source code instrumentation is an approach where instrumentation statements, such as annotations or method calls to the coverage calculation tool, are added to the source code. The code is then compiled to produce an instrumented assembly.

2. Intermediate code instrumentation is an approach where the compiled class files are instrumented by adding new byte codes, and a new instrumented class is generated. 
3. Run-time information collection is an approach that collects information from the runtime environment, as the code executes, to determine coverage information.

As the program under test is executed, coverage is measured according to one, or a combination, of these measuring approaches.

This thesis uses run-time information collection, in the form of execution tracing, to measure code coverage. Execution tracing involves closely monitoring the Java Virtual Machine (JVM) that executes a program, through the use of the Java Platform Debugger Architecture (JPDA) $[4]$.

\subsubsection{JUnit Test Generation}

The symbolic state, constructed during symbolic execution, contains sufficient information, such as local variable values and path conditions on symbolic values, to generate a test case that will reach that specific symbolic state during normal execution. It is, therefore, possible to generate test cases to execute specific branches of a program, with the help of the symbolic states that represent those specific branches. These test cases are generated as JUnit test cases, which can be compiled by the Java compiler, and executed with the JUnit testing framework.

\subsection{Literature Synopsis}

James King [33] sought to find a middle-ground between program verification and program testing in 1976. This middle-ground was symbolic execution. It presented a way to symbolically represent the behaviour of a program. Symbolic execution has since been used in a variety of studies, to investigate how the formal method can assist in the analysis and testing of programs.

The research most relevant to this thesis, and the symbolic execution engine used in this thesis, is that of Tomb et al. [39]. Tomb et al. studied the use of symbolic execution to detect unhandled run-time exceptions. They analysed a program's byte code to detect any possible exceptions that may occur during the execution of a program. If this exception is not properly handled, e.g., with the help of try-catch blocks in Java, a JUnit test case is generated to confirm whether the possible exception is, in fact, handled or not.

Many studies regarding symbolic execution aim to use it to generate JUnit test cases to achieve high levels of code coverage [45]. The studies have shown that symbolic execution can 
be used to generate such test cases from an analysis of the code; the code is analysed, and a separate, independent test suite is created. However, none of the studies found considered an already existing JUnit regression test suite.

Code coverage calculation tools are wide-spread, and measure a variety of code coverage criteria. Those that are available in the industry are typically focused on measuring common coverage criteria, such as statement (line) coverage, branch coverage, method coverage, and class coverage [46]. The less common coverage criteria are typically omitted due to the increased complexity and difficulty to measure them.

\subsection{Objectives}

The goal of this thesis is to explore the practical applicability of using the static analysis formal method known as symbolic execution, to calculate code coverage of an existing regression test suite, and then to extend the test suite by generating additional test cases, such that the resulting regression test suite is as thorough and efficient as possible.

The main objectives of this thesis are as follows:

- Use symbolic execution to measure code coverage of an existing regression test suite for various common coverage criteria. These common criteria include statement coverage, branch coverage, class coverage, and method coverage.

- Use the results from symbolic execution and coverage calculation to generate JUnit test cases for all areas not covered by the current regression test suite. Evaluate the effectiveness of the resulting regression test suite.

- Design the solution to benefit from a multi-processor environment, and evaluate the benefits of this concurrent design.

- Integrate the solution into an Eclipse plug-in to improve its usability.

\subsection{Contributions}

To achieve the objectives of this thesis, ATCO (Automated Test Coverage Calculation and GeneratiOn) was developed, and the following contributions were made: 
- Symbolic execution can be used to measure code coverage of the common coverage criteria when primitive data types are used. A level of inaccuracy is introduced with more complex data types and branching conditions, however, since the path conditions containing complex data types cannot currently be solved.

- Combining symbolic execution with coverage calculation allows JUnit test cases to be generated automatically for areas of a program not tested with the current regression test suite.

- $A T C O$ is designed to utilise the additional processors of a multi-core platform. This allows sections of $A T C O$ to execute concurrently, thereby reducing the time required to perform its tasks by up to $54 \%$.

- $A T C O$ is implemented as an Eclipse plug-in, utilising the graphical user interface (GUI) extensions, provided by Eclipse. This greatly improves the usability of ATCO.

Along with the main objectives, the following additional contributions were made during this thesis:

- Using symbolic execution to measure code coverage of a less common coverage criterion, such as loop coverage.

- Extending ATCO to perform test redundancy detection, i.e., monitoring whether the tests in the existing regression test suite cover unique areas of the code, or whether they only cover areas covered by other tests.

- Using execution tracing to calculate coverage for the manual execution of a program, which can give program testers the ability to verify the thoroughness of their manual testing.

\subsection{Outline}

The rest of this thesis is structured as follows.

Chapter 2: Theoretical Background provides an overview of the theory of testing and test adequacy. This chapter is separated into two parts. 
First, the chapter presents two methodologies of testing, the one is proving the absence of bugs, and the other is finding bugs. The chapter also presents two approaches to these methodologies, namely program verification and program analysis. These approaches, and how they are used with regard to testing, are compared. This broad overview provides the necessary context for an in-depth description of symbolic execution, and how it can be used for testing.

Second, the chapter presents an overview of test adequacy. It describes the use of testing requirements in the measurement of test adequacy, followed by a classification of various test adequacy criteria categories. The chapter then discusses code coverage, a field of study under test adequacy, and particularly focuses on program-based coverage, where the coverage criteria are described. Available methods of measuring coverage are also presented, followed by a discussion on some of the coverage calculation tools, and their preferred methods of measuring coverage.

Chapter 3: Design and Implementation contains an in-depth description of the design and implementation $A T C O$, the Eclipse plug-in that is implemented for this thesis. This description includes how symbolic execution is used, how that information is presented in preparation of coverage calculation, how coverage is measured, and how the resulting data is used to automatically generate JUnit test cases. Additionally, a description of Eclipse's extensible plug-in framework, and the design of the Graphical User Interface (GUI) for ATCO, as it is integrated into the Eclipse environment, is discussed.

The chapter also discusses the benefits, and some interesting occurrences, of using symbolic execution to measure coverage, as well as the benefits of using execution tracing to drive the coverage calculation. Various possibilities of extending the functionality of ATCO are presented. These extensions are: measuring uncommon coverage criteria, test redundancy detection, and method invocation recording.

The concurrent design of $A T C O$, the design considerations that impact the concurrent measurement of coverage, and additional optimisation considerations are also discussed.

Chapter 4: Evaluation presents and analyses the various experiments that are used to evaluate $A T C O$.

The experiments are separated into three sets. First, the experiments are aimed at verifying the correctness of $A T C O$, through manual inspection as well as a comparison with another 
coverage calculation tool. Second, the experiments focus on evaluating the performance benefits of the concurrent design of $A T C O$. Third, the experiments measure the effectiveness of the resulting regression test suite. Each set of experiments begins with a discussion on the goals of that set of experiments. This is followed by a description of the programs that are used for those experiments. And each set of experiments is concluded with an analysis of the results achieved by that set.

Chapter 5: Conclusion concludes this thesis by summarising the findings in using symbolic execution to measure code coverage, and then automatically generate JUnit test cases. It also presents potential avenues for future work. 


\section{Chapter 2}

\section{Theoretical Background}

\subsection{Introduction to Program Verification and Program Analy- sis}

Developing large-scale, complex systems has become common practice in software development. Unfortunately, an increase in scale and complexity often also increases the likelihood of subtle errors being introduced into the system. When a program does not function according to its intended design and purpose it may result in an undesirable user experience, financial losses may be incurred and, in some cases, human lives may even be lost [8]. Testing, as will be discussed in this chapter, has, therefore, become an important part of the software development cycle.

Testing aims to prevent, or at least minimise, the damage caused by erroneous systems through one of two methodologies. The first methodology is proving the absence of bugs. In order to prove the absence of a bug, it needs be proven that the bug can never occur on any of the execution paths of the system. The second methodology is finding bugs, e.g., finding code that has been implemented incorrectly due to, e.g., an insufficient understanding of the programming language, or as a result of insufficient error checking for irregular inputs.

In this chapter, program verification and program analysis and how these methods may be used to test a system to either find bugs, or to prove the absence of bugs will be briefly discussed. Some of the techniques in these testing methodologies that are applicable to this thesis, will also be discussed. 


\subsection{Program Verification}

Program verification is a formal method. Formal methods are mathematically-based languages, techniques, and tools for specifying and verifying complex software systems. To verify a program, the system and the properties to be verified are expressed in some formalism and the verification techniques are, subsequently, used to analyse the system to determine whether the desired properties have been retained in the program.

Two well-established verification techniques are model checking and theorem proving (Clarke et al. [8]).

\subsubsection{Model Checking}

Model checking is a technique for verifying finite state concurrent systems. This technique was initially used for hardware verification, but later much research went into also using model checking to analyse the specifications of software systems [8].

The model checking process consists of three tasks:

Modelling: To verify the design of a system, it needs to be converted to a model that can be read by a model-checking tool. A model is generally constructed using a modelling language, e.g., the Promela modelling language used for the model checker, SPIN. Alternatively, the implementation may be verified by using a model-checking tool that supports the programming language in which the system has been written. Depending on the size of the state space and the limitations on time and memory, abstractions may be required, to exclude irrelevant details.

Specification: The correctness properties that should be satisfied during program execution, need to be specified. Temporal logic is commonly used to specify the correctness properties, since it is able to determine how the behaviour of the system evolves over time.

Verification: The model checker automatically verifies whether the system satisfies the specified properties, by traversing the state space and determining whether the properties have been retained in the model. If the verification process fails to terminate due to the size of the model, the model needs to be adjusted, e.g., by adding additional abstractions, and the verification process needs to be restarted. In the event that the verification 
process terminates normally and an error has been encountered, the error trace needs to be examined manually. This error trace, known as a counterexample, may be used to determine where the error occurred. Errors may indicate one of two possibilities:

1. An error in the design of the system, i.e., one or more properties are not satisfied in the design. Examining the error trace can assist in locating the problem area in the design, allowing it to be repaired.

2. Human error in either the modelling of the design, or the specification of the properties. Errors that occur as a result of human error are often called false negatives, denoting an error that occurs in the model that will not occur in the design. Examining the error trace can assist in identifying the problem.

The most significant drawback of model checking is the state-explosion problem [7]. The state-explosion problem is defined by the rapid increase in the size of the state space as system complexity increases, i.e., the state space grows exponentially with each program variable, concurrent component, and communication channel in each process running on the concurrent system. Since resource requirements and verification time are directly proportional to the size of the state space, the availability of these resources limits the size and complexity of the systems that may be verified.

\subsubsection{Theorem Proving}

In model checking, a system is modelled as program states at specific intervals and the properties to be checked are specified using a formalism such as temporal logic. In theorem proving, however, a program is modelled as a set of mathematical definitions in some formal logic and the properties to be checked are then derived as theorems that follow from these definitions [3].

Because the program is modelled as a set of mathematical definitions, theorem proving can verify programs with infinite state spaces, as well as handle complex data types and recursion, effectively. This makes theorem proving well suited for "data-intensive" systems with complex data structures [35].

Even though theorem provers have some distinct advantages over model checking, they do have significant disadvantages, as well. First, the generated proofs can be large and difficult to understand. As a result, the generated proofs require a great deal of user expertise and effort 
to use. Second, theorem provers cannot be fully automated, thereby, again, indicating the need for user expertise [35].

\subsubsection{Program Verification Tools to Prove the Absence of Bugs}

To prove the absence of a bug, with respect to the specified properties, the model checker has to exhaustively investigate all execution paths to prove that no execution path violates the specified properties.

Model checking is a strong and successful formal method for verifying a system. However, original model checkers required the specification of system design in a modelling language, which required expert knowledge.

Corbett et al. [11] attempted to solve the problem of converting a system to be model checked to a modelling language, by creating Bandera. Instead of requiring expert knowledge to create models from existing systems, this tool, Bandera, accepts Java source code and converts it into a modelling language that is accepted by model-checking applications. To minimise the model size, developers have to manually add data abstraction information.

\subsubsection{Program Verification Tools to Find Bugs}

Some model checkers have been adapted to handle programming languages. The model checker, $S P I N$, has been adapted to handle embedded $C$ or $C++$ in verification models [22].

Visser et al. [43] took another path and created Java Pathfinder (JPF), a custom-designed model checker. JPF is a model checker designed specifically for testing multi-threaded, interactive Java programs. It works from the compiled version of Java source code, known as byte code, which is executed within the Java Virtual Machine ( $J V M)$ run-time environment. The model is constructed by using a custom $J V M\left(J V M^{J P F}\right)$ that analyses the byte code and interprets the behaviour of the different threading components of the program, thereby constructing the state space. $J V M^{J P F}$ guides the execution of the program while JPF analyses the resulting state space to find race conditions and deadlocks that are common in multi-threaded programs.

\subsubsection{Conclusion}

Program verification may either be used to prove that specific correctness properties are satisfied within all existing execution paths of a system, or it may be used to find behavioural bugs in 
a system.

It is important to note that even if a model checker could perform an exhaustive verification, i.e., check that all execution paths satisfy all the specified properties, it still does not guarantee the correctness of the system. Verification techniques check only for the specified properties. If there were an error in the system that would have violated any particular property, which had never been specified and, therefore, never been checked, that particular error would remain undetected. Verification techniques also have a scalability issue, due to the state explosion problem. However, program verification increases our understanding of a system by revealing inconsistencies, ambiguities, and any incomplete sections of a system that might otherwise have been undetected [8].

\subsection{Program Analysis}

Another approach to testing is program analysis. Program analysis, often referred to as static analysis, offers static compile-time techniques for predicting safe approximations of program data or program behaviour that arise dynamically at run-time, during the execution of a program on a computer. According to Nielson et al. [34], program analysis may be divided into a number of techniques, with this thesis focusing on the following three techniques:

1. Data-flow Analysis graphically represents data changes in a program, where the nodes represent possible states of the data and the edges represent state changes.

2. Constraint-based Analysis is used to approximate control-flow data. Control-flow analysis determines information about execution behaviour, i.e., determining which statements lead to which other statements. However, in functional and object-oriented languages, this information is not, necessarily, available immediately. Constraint-based analysis allows for the control-flow analysis of these languages, by representing data as sets of constraints.

3. Abstract Interpretation: Whereas data-flow analysis represents changes in the state of data, abstract interpretation represents the state of data at a specific point in the program by using collecting semantics to accurately trace the possible values of data at that point. Galois connections [34] are then used to group these collecting semantics for 
each field of data, thus, obtaining a smaller domain that represents the behaviour of the data of the program more accurately.

There are various sub-techniques that further define these techniques [34]. For the purposes of this thesis, particular attention is paid to two of these sub-techniques that form part of data-flow analysis. These sub-techniques are intraprocedural and interprocedural analysis. The defining difference between these two techniques is the manner in which they handle methods in a program.

Intraprocedural Analysis analyses each method of a program as an independent block of code. Any function or procedure call, encountered during intraprocedural analysis is skipped, thereby ignoring the effect that such calls might have on the data. By skipping these calls, the approximations of data values are not as accurate, but require significantly less memory compared to interprocedural analysis, since each method is handled as a closed system.

Interprocedural Analysis takes function and procedure calls into account. All the function and procedure calls encountered are followed, thereby taking into account the effect they have on the data. This approach allows for a more accurate analysis of the program than intraprocedural analysis, but demands more resources and gives rise to a number of complications. These complications arise as a result of the need for ensuring that calls and returns match one another, when dealing with parameter mechanisms (and the aliasing that may result from call-by-reference method parameters), and when allowing procedures as parameters. Resource requirements can be reduced by defining a call depth, $k$. The purpose of $k$ is to limit the depth that the analysis will follow from the top-level method. Thus with $k=1$, all procedure calls within the analysed method will be called, but calls within those procedures will not be called. The higher the value of $k$, the deeper the analysis will follow the call graph, but the more memory will be required when applying this technique.

King [32] created a formal method of static analysis, called symbolic execution, which relies on constraint-based analysis, as well as intraprocedural and interprocedural analysis, mentioned above. This is the analysis technique this thesis is based on, and it will, therefore, be discussed in detail later in this chapter. 


\subsubsection{Program Analysis Tools to Prove the Absence of Bugs}

Analysis tools that focus on proving the absence of bugs are path-insensitive, i.e., the analysis is not guided by execution paths and conditional branches. ASTREE [12] is an abstract interpretation-based program analyser that focuses on proving the absence of run-time bugs in $C$. It uses data-flow analysis to verify that all data in the program is used within the rules defined by the programming language, e.g., ensuring the short variable type is used only within the constraints of its domain, or that array indexing occurs within the defined bounds. ASTREÉ uses multiple abstract domains to analyse different aspects of the program, as well as to allow the analysis processes of these domains to closely interact with each other to achieve mutual reduction over all domains.

Another example of an analysis tool is ESC/Java [19], a tool that uses annotations to indicate design decisions of routines. These annotations are inspected during intraprocedural analysis and any violations found against these design decisions are reported. ESC/Java uses a theorem-prover to reason about program semantics and is, therefore, able to check for synchronisation errors of concurrent programs. The annotations need to be added manually, so a substantial period of time is required for development preparation.

\subsubsection{Program Analysis Tools to Find Bugs}

Analysis tools focusing on finding bugs are typically path-sensitive, i.e., the analysis is guided by execution paths and/or conditional branches. An example of such an analysis tool, is the static analyser FindBugs, created by Hovemeyer et al. [23]. This analysis tool is built on the assumption that most bugs that reside in programs are blatant errors that are easy to find during code inspection. FindBugs uses class structure, data-flow, and control-flow information, as well as an instruction state machine to analyse each class and search for patterns that typically cause errors during execution. It uses simple, broad techniques to uncover common, and likely, bugs. FindBugs also requires little or no development preparation for its application.

\subsubsection{Conclusion}

We discussed static analysis and how it may be used to both analyse and verify a program through code-inspection methods, using information extracted from the code by means of data-flow and control-flow information, or by using information supplied by the user, by means 
of annotations. However, static analysers are unsound, i.e., they return false positives. If a tool returns a false positive, it means that the tool has indicated that a section of code contains an error, but, that under execution, it is not an actual error. Since the user has to go through all identified errors and determine whether a bug actually exists, examining these false positives are usually time-consuming.

\subsection{Symbolic Execution}

Symbolic execution is the formal method that forms the fundamental part of this thesis.

In 1975, James C. King [32] identified program testing and program proving as the two extremes of program verification in terms of the number of times a program needs to be executed. On the one extreme, to verify the correctness of a program through testing, the program must be executed at least once for every possible unique input, which, typically, requires an infinite number of program executions. On the other extreme, to verify the correctness of a program through a correctness proof, the program requires no execution, but a tedious, and often difficult, formal analysis. King proposed a middle-ground in the spectrum, between running individual tests and general correctness proofs. This proposed middle-ground is known as symbolic execution [33], which is the analysis technique that forms the fundamental part of the research in this thesis.

\subsubsection{Concept}

The notion of symbolic execution follows naturally from normal execution. The code is inspected line-by-line, much like an interpreter would execute machine code. However, instead of actually executing the code, the execution of the program is simulated by inspecting the code and maintaining a representation of the static state of the program. This representation of the static state will, from here on, be called the symbolic state. The symbolic execution of a program must be structured in such a way that the symbolic state is equivalent to the static state of the program, during normal execution.

When a procedure is symbolically executed, execution proceeds as normal until any data, external to the procedure, is accessed. Data external to the procedure are data entities, like parameter variables of the procedure, global variables in the program, Input/Output operations, 
and others. Whenever an external data entity is accessed, that entity is regarded as a symbolic value. These symbolic values are used to represent some unknown, yet fixed value, as opposed to, e.g., program variables, which are symbolic names that may assume numerous different values during the execution of a program. Values that are not external to the procedure are known as concrete values, and include local variables with non-symbolic values assigned to them, or constants. From this description, it is clear that symbolic execution is, by its definition, an intraprocedural analysis technique.

There are two situations where the symbolic state will manipulate data differently from normal execution. These two situations are evident during the computation of expressions and during conditional branching.

\section{Computation of Expressions}

Since the symbolic values are unknown, any expressions containing symbolic values cannot be solved. Instead, these expressions are represented in terms of the symbolic values within them. The example shown in Figure 2.1, p. 19 explains this representation clearly.

\begin{tabular}{|c|c|c|}
\hline & Expression & Symbolic Representation \\
\hline$A$ & $=\alpha$ & $A=\alpha$ \\
\hline$B$ & $=\beta$ & $B=\beta$ \\
\hline$C$ & $=A+2 \times B$ & $C=\alpha+2 \times \beta$ \\
\hline$D$ & $=C-A$ & $\begin{aligned} D & =\alpha+2 \times \beta-\alpha \\
& =2 \times \beta\end{aligned}$ \\
\hline
\end{tabular}

Figure 2.1: This is an example of the symbolic representation of expressions. In this example, variable $A$ is assigned the symbolic value $\alpha$ and variable $B$ is assigned the symbolic value $\beta$. Variables $C$ and $D$ are assigned values through arithmetic expressions composed of variables $A, B$ and $C$, and the resulting representation of its assigned value is shown under the Symbolic Representation.

\section{Conditional Branching}

Conditional branching in a program occurs at every branching statement, such as an if statement, where only one of the paths is followed given the conditions in the if. During symbolic execution, concrete conditions, i.e., conditions composed of only concrete values, are handled normally, since the result of the condition can be calculated and the correct execution path can be followed. 
Since symbolic values are unknown, if statements, with one or more symbolic conditions, cannot be resolved during symbolic execution. Therefore, these conditional statements are referred to as unresolved conditional statements. Whenever an unresolved conditional statement is encountered, it has to be assumed that the condition can be both true and false. This occurs, because it has to be assumed that a symbolic value can represent any value within the domain of its type, i.e., if the symbolic value is of type int, it can assume any value in the domain $-2^{31}<=S V<=2^{31}-1$. Since the condition has to be assumed as both true and false, both execution paths need to be followed. When both paths are followed, it means that the execution forks into two parallel executions of the current procedure, where the execution of each path proceeds as normally, except for the assumed outcome of the unresolved conditional statement.

The assumptions are stored with their values in the symbolic state as a path condition, because assumptions made by one unresolved conditional statement may assist in resolving subsequent unresolved statement executions.

\subsubsection{Path Condition}

The path condition $(P C)$ is an accumulator of conditions on symbolic values which determines a unique control path through the program. Each path has its own $P C$ and no two $P C$ s are ever identical.

At the beginning of a program, the $P C$ is set to true. When a conditional branching statement is encountered, its condition (represented by $b(C)$ ) is examined. If $b(C)$ contains no symbolic values, i.e., only concrete values, then $b(C)$ can be solved using the concrete values. The correct path can, then, be followed with no modification to the $P C$. However, if $b(C)$ is an unresolved condition, the execution has to be forked into two parallel executions. The two forked executions will each receive a copy of the $P C$, at that point, and the assumed result of $b(C)$ is added to the $P C$ :

$$
\begin{aligned}
& P C_{1} \equiv P C \wedge b(C) \\
& P C_{2} \equiv P C \wedge \neg b(C)
\end{aligned}
$$

Execution of each path continues in parallel, where the path represented by $P C_{1}$ assumes $b(C)=$ true and the path represented by $P C_{2}$ assumes $\neg b(C)=$ true. Figure 2.2, p. 21 provides an example taken from [31]. 


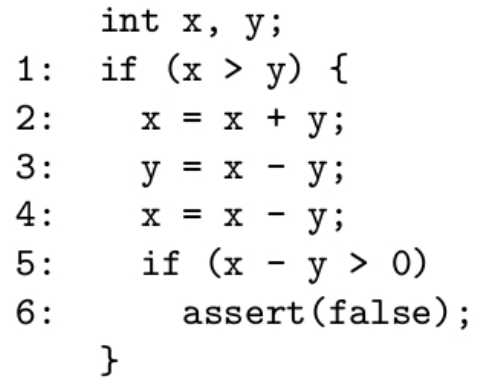

int $\mathrm{x}, \mathrm{y}$;

1: if $(\mathrm{x}>\mathrm{y})\{$

$x=x+y$

$\mathrm{y}=\mathrm{x}-\mathrm{y}$

$\mathrm{x}=\mathrm{x}-\mathrm{y}$

5: $\quad$ if $(x-y>0)$

\}

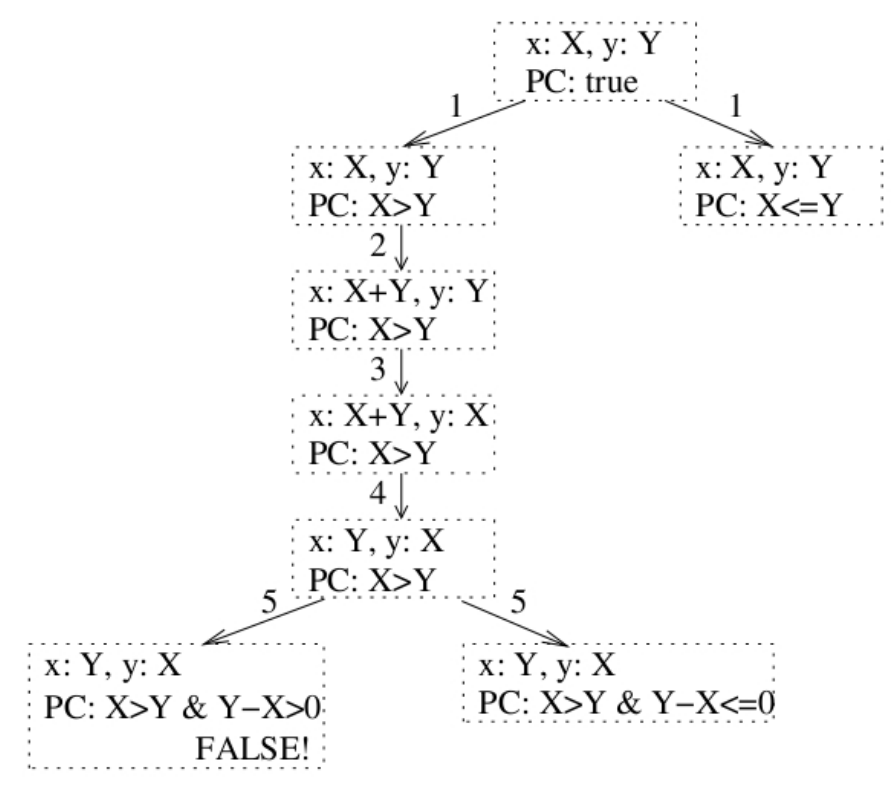

Figure 2.2: This figure contains a code and graph example of path conditions. The code snippet on the left shows two variables, $\mathrm{x}$ and $\mathrm{y}$. These variables have the symbolic values $\mathrm{X}$ and $\mathrm{Y}$, respectively, associated with them. The graph on the right shows how the $P C$ changes during symbolic execution. The number, associated with each edge, shows the line number of the statement executed, while each node represents the state of the $P C$ and the symbolic variables.

\subsubsection{Symbolic Execution Tree}

As is the case with normal execution, where the static states may be used to produce an execution tree that represents the different execution paths that the program may take, so can symbolic states be used to produce a Symbolic Execution Tree (SET) that characterises the execution of a procedure. Each node in the SET represents the program state after the program statement that has been executed, and the transition between nodes is a directed arc connecting the two nodes. Each unresolved conditional statement that causes the symbolic execution to be forked into two parallel execution paths, results in a fork in the SET.

Each SET node typically contains:

- a statement counter

- variable values stored as concrete values, if concrete, or as formulae over symbolic values, if symbolic.

- a $\boldsymbol{P C}$ consisting of a set of constraints over symbolic values characterising conditions the 
variable values would have to satisfy to reach the node.

The graph in Figure 2.2, p. 21 is an example of what a $S E T$ would look like for the code snippet provided in the figure. Another example of a code snippet and its corresponding SET, taken from [25], is shown in Figure 2.3, p. 22 and Figure 2.4, p. 23 respectively.

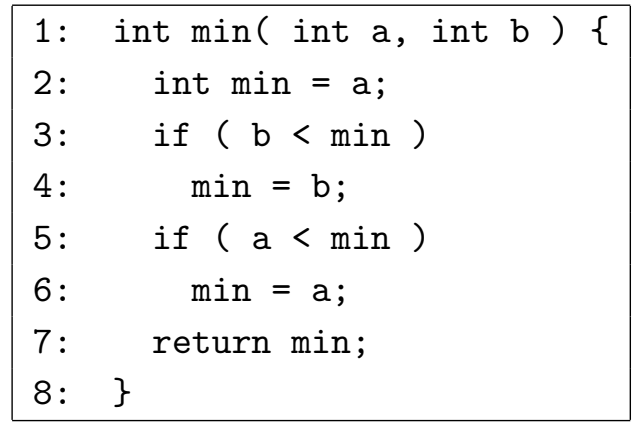

Figure 2.3: A simple method example, which is used to illustrate a SET. The method calculates the minimum of two arguments.

\subsubsection{Issues}

Symbolic execution has issues that need to be addressed. First, it is possible for a path condition $(P C)$ to be unsatisfiable, i.e., it may contain contradicting conditions [16]. The example in Figure 2.2, p. 21 illustrates this situation. In the example, the assert (false); statement indicates that, at that point, a contradiction would be present in the $P C$. The code snippet switches the symbolic values of the variables, i.e., $\mathrm{x}$ becomes $\mathrm{Y}$ and $\mathrm{y}$ becomes $\mathrm{X}$. The first condition in the $P C$ is that $\mathrm{X}>\mathrm{Y}$, while the second condition states that, to follow that execution path, $\mathrm{Y}-\mathrm{X}>0$, i.e., to follow that execution path $(\mathrm{X}>\mathrm{Y} \& \mathrm{Y}-\mathrm{X}>0)$ must be true, which is impossible. Although the code is semantically correct, the instruction at line 6 will never be reached, due to the contradicting conditions of the variable $\mathrm{x}$. Since symbolic execution will attempt to fork the execution at line 5, a theorem-prover will need to be instrumented to ensure that the $P C$ s do not contain contradictions. All $P C$ s with contradictions indicate an execution path that will never be followed during normal execution and, therefore, the execution path should not be expanded. Figure 2.4, p. 23 shows a SET with two paths that contain contradictions. These two paths are the paths that end with nodes labeled backtrack. 


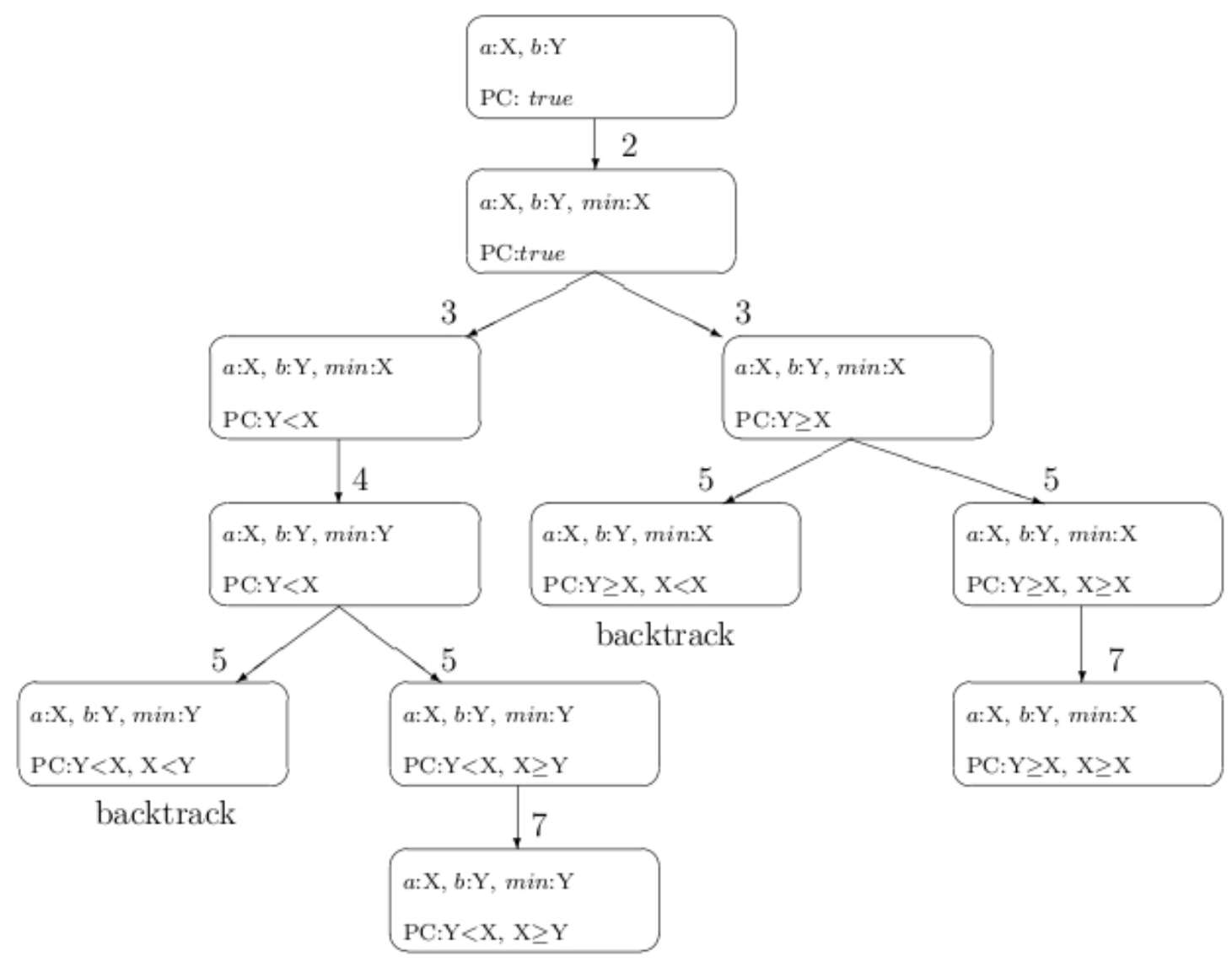

Figure 2.4: This is the $S E T$ graph of the method displayed in Figure 2.3, p. 22.

Second, the number of times a loop with symbolic conditions needs to be iterated is unknown. Concrete loops, i.e., loops that contain only concrete conditions, are straightforward during symbolic execution. A concrete loop is merely a block of code that will be executed a concrete number of times. Thus, during symbolic execution, the code is symbolically executed a concrete number of times. However, limiting the number of iterations of concrete loops might be considered if the concrete number becomes large. Symbolic loops, i.e., loops that contain symbolic conditions, need to be handled differently. The conditions contain symbolic values, requiring that both the true and false paths be followed, at every iteration of the loop. The true path of a loop results in another iteration, while the false path results in the execution breaking out of the loop. In other words, after every iteration of the loop, the loop is both exited and re-entered for another iteration. This can result in the loop iterating through the entire domain of the counting variable's type, depending on the start value and whether counting is incremental or decremental. As the symbolic execution will attempt to 
iterate through the entire domain of the counting variable's type, a state explosion problem may occur. The explosion problem becomes even more apparent when nested symbolic loops are used, i.e., symbolic loops containing symbolic loops.

The example code snippet and resulting SET illustrating this problem are shown in Figure 2.5, p. 24 and Figure 2.6, p. 25, respectively.

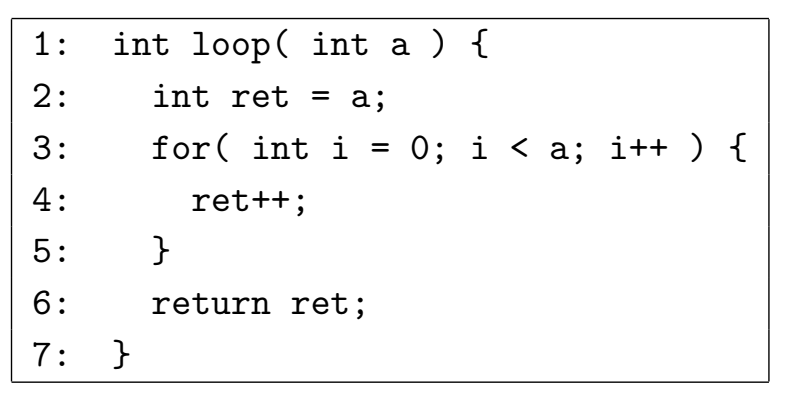

Figure 2.5: This method shows a simple example of a loop with symbolic conditions. The resulting $S E T$ is shown in Figure 2.6, p. 25.

It is, therefore, necessary to limit the number of iterations of a loop to adequately test the body of the loop, while preventing a state explosion or unnecessary execution. A symbolic loop could iterate three times or, even, three million times during normal execution, but the actual number of iterations is never known during symbolic execution. Limiting the number of iterations will, therefore, either result in certain execution paths not being followed, or, for certain execution paths that will not exist during normal execution to be followed.

\subsubsection{Advantages}

Symbolic execution is a very diverse, formal technique that has been proposed for many verification and testing activities such as symbolic debugging, test data generation, verification of program correctness and program reduction [10].

Symbolic execution requires no modification to the code such as reformatting or preparation such as annotations, in order to analyse it effectively. This makes any analysis tools, based on symbolic execution, very easy to use. Also, since it simulates normal execution, the behavioural information extracted from symbolic execution, closely resembles the behaviour of a program during normal execution, thereby representing the behaviour of a program more accurately than by means of regular static analysis. 


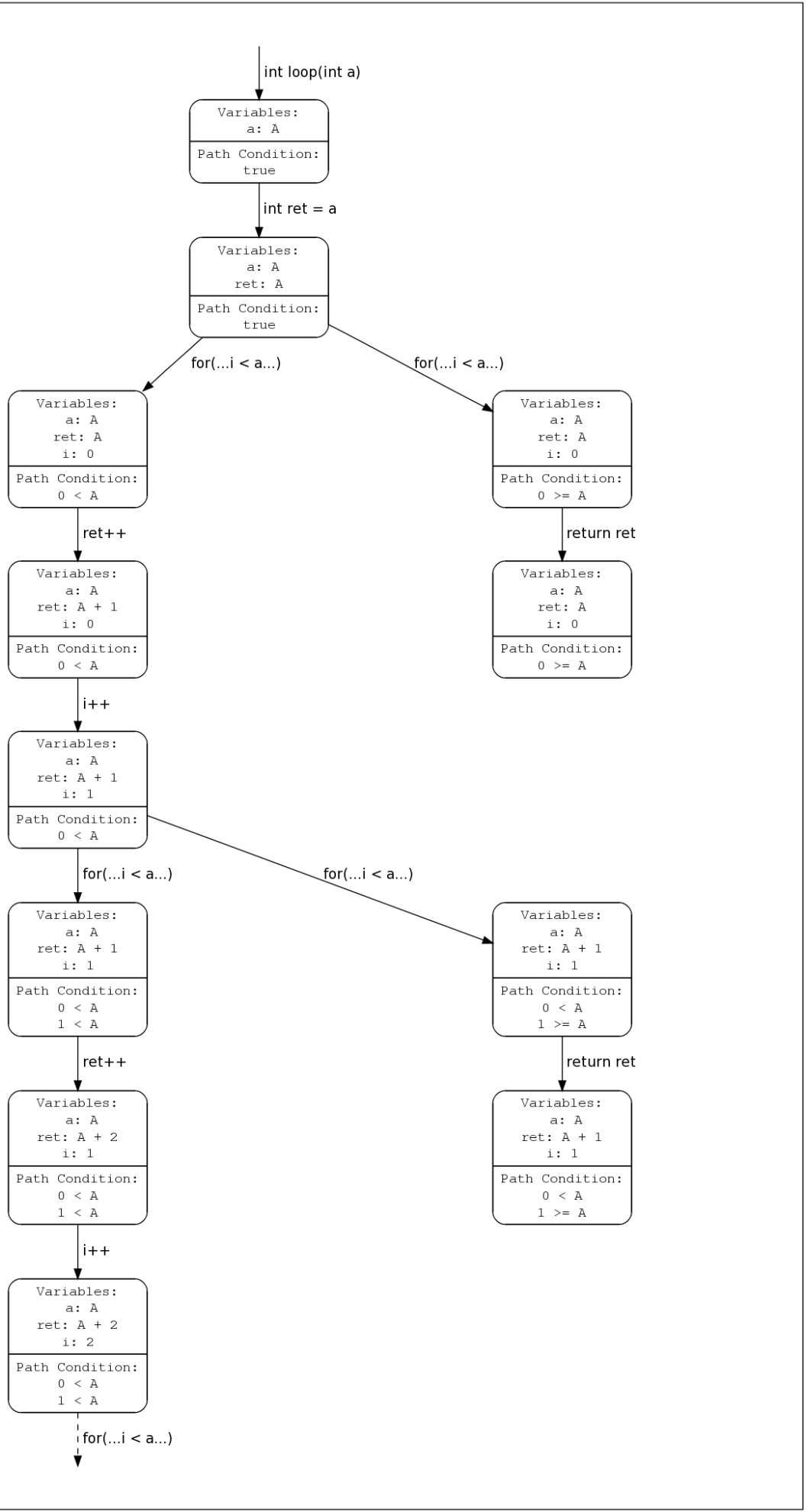

Figure 2.6: This is an example of a symbolic loop, represented as a $S E T$. It is the result of the code in Figure 2.5, p. 24 being symbolically executed. Each node represents a symbolic state with variables and path conditions. Each edge indicates the Java statement that was executed to transition from the one node to the other. 


\subsubsection{Extending Symbolic Execution} Interprocedural Symbolic Execution

As mentioned earlier, symbolic execution is, by nature, intraprocedural, i.e., every procedure is analysed as an individual entity with no link to any other procedure. Tomb et al. [39] researched the notion of interprocedural symbolic execution. According to the findings of Tomb et al. the level of interprocedural analysis has no noticeable effect on the discovery of errors, but it does assist in more constrained $P C$ s and, thus, more accurate representations of the constraints on symbolic values.

Tomb et al. mention that object-oriented programs using accessor methods rarely access instance fields directly. As a result, any value returned from a procedure call or an instance field accessed by means of an accessor method, will result in the creation of a new symbolic value. Tomb et al. hypothesise that setting the interprocedural analysis level to one, would yield a significant immediate benefit, by making it possible to reason about field values. However, interprocedural symbolic execution may cause $P C$ s to contain contradictions, thereby indicating the existence of infeasible execution paths. This gives rise to the need for pruning such infeasible paths from the SET.

\section{Path Pruning}

When a program is compiled, the code is not checked for contradictions in conditional branches. It is, therefore, possible for infeasible paths to be present in the code. Symbolically executing these paths would therefore be unnecessary, since they will never be executed during normal execution of the program. Infeasible paths can therefore be reported, but not explored, which reduces the resource requirements for analysing the program. During experimentation, Tomb et al. [39] discovered that pruning infeasible paths pruned less than $10 \%$ of paths during intraprocedural analysis, while pruning $20 \%$ of paths during interprocedural analysis, and up to $50 \%$ of paths with larger examples [39]. Path pruning, therefore, becomes indispensable during interprocedural analysis.

Infeasible path pruning also increases the execution time if a $P C$ has no obvious contradictions. An obvious contradiction is the situation where at least two conditions in a $P C$ are exact inverts of one another. These can easily be identified without using a decision procedure. 
A non-obvious contradiction requires a decision procedure to identify the contradiction, which is an expensive computation. As an example, consider symbolic integer variable A:

$$
\begin{array}{ll}
\text { obvious contradiction: } & P C=A<2 \& A>=2 \\
\text { non-obvious contradiction: } & P C=A<2 \& A>4
\end{array}
$$

\subsubsection{Symbolic Execution in Practice}

Symbolic execution has various practical uses with respect to the role it plays in analysis and design tools. Some examples of its application will be discussed in this section.

Tomb et al. [39] use symbolic execution for testing and test case generation. They attempt to detect unhandled run-time exceptions in Java byte code by deciding the satisfiability of arbitrary formulae in first order logic. These detected exceptions are then recreated by solving the $P C$ to reach the state where the exception might occur. The solved values are then used to generate test cases using the Java Reflection API in an attempt to verify the validity of the error.

Siegel et al. [37] use symbolic execution to assist in the parallelisation of sequential programs, focusing, specifically, on parallel numerical programs. According to them, the process of "parallelising" a sequential program is notoriously difficult and error-prone. In the first instance, the main issues are that it is infeasible to test more than a tiny fraction of possible inputs that a numerical program will encounter during execution. Therefore, it is impractical to attempt to show that a program behaves correctly on all possible inputs. Second, in concurrent programs, unlike in sequential programs, the order in which events occur differs with every execution, due to a number of factors, such as the load on the processors, and the latency of the communication network. Symbolic execution is therefore used to represent the outputs of the program as constraints on the inputs, i.e., the $P C \mathrm{~s}$ of the outputs. These $P C \mathrm{~s}$ of the parallel and sequential versions of the program are then used together with a model checker, like SPIN, to explore the possible states of the parallel and sequential programs. If the results produced by the parallel program agree with the results produced by the sequential program, then the two programs must be equivalent.

Dynamic invariant inference tools like Daikon [16] observe the behaviour of program properties during the execution of a test suite. These properties are a collection of object state invariants, method pre-conditions, or method post-conditions, which are collectively known 
as invariants. However, according to Csallner et al. [16], these dynamic invariant inference tools are likely to generate either incorrect, or irrelevant, invariants. Therefore, they combine symbolic execution with the process of dynamic invariant inference. The invariants are generated during the execution of a test suite and the source code is symbolically executed, as well. The $P C$ s generated through symbolic execution are then used, together with the invariants generated through dynamic invariant inference, to determine which of the invariants are relevant.

According to Coen-Porisini et al. [10], systems such as aircraft avionics systems, nuclear power plant control systems, and patient monitoring systems, which are all safety-critical systems, need to be highly reliable, since failures in these systems may have catastrophic consequences. However, while formal methods successfully enhance the quality of software, they are often neglected in practice. Industrial software project verification usually relies on techniques that perform code inspection or testing to find programming errors, but correctness is seldom formally verified. Coen-Porisini et al. designed a Path Description Language (PDL) which is used to express safety properties as predicates of execution paths. Symbolic execution is used to construct operational models of the software on which the safety properties can be assessed. Assessing the safety properties on the generated operational models can, therefore, verify whether or not the specified safety property is retained in the safety-critical system.

\subsection{Dynamic Analysis}

The program analysis section showed that static analysers were unsound, since all errors that were found, were reported without any validation on a running instance of a program. Dynamic analysis follows more naturally on manual testing. Dynamic analysis is built on the concept of, first, black-box testing, where the tester tests the entire application without any knowledge of its implementation, and, second, static regression testing, where the developer maintains a test suite that executes different pieces of overall functionality [13].

Where static analysers test programs through code inspection, dynamic analysers, also known as run-time analysers, test programs by executing the programs. This is done by running a series of tests against the program and seeing whether any errors occur during execution. The problem with this, however, is that the time it takes to create and maintain these test suites 
can be substantial, especially for applications near the end of the development cycle.

Csallner et al. created a tool called JCrasher [13] to assist in avoiding the above-mentioned problem. JCrasher is a random test tool for Java classes. It automatically generates a random number of tests that are type-correct, but contain random data inputs. These tests are run against the program and any errors that occur during execution are reported. Random testing, which is a form of blind testing [13], investigates the capability of a program to handle unexpected scenarios, i.e., the robustness of the program. It requires minimal user input, easily covers shallow boundary cases, like incorrect inputs on arithmetic functions, and effectively checks whether pre-conditions of public methods are enforced. However, since the inputs are all random, the overall effectiveness of testing is proportional to the number of tests generated and executed.

It is important to note that when using dynamic analysis, the static state of a program becomes relevant. Each test that is executed against a program can affect the static state of the program, which, in turn, could affect the outcome of other tests. Precautions need to be put in place to preserve the static state which would, in turn, ensure the integrity of each test.

\subsubsection{Focused Testing using Program Analysis}

Static analysers are effective at finding coding bugs in a program, but as mentioned earlier, they are prone to producing large amounts of false positives, as well as producing reports that may be difficult to understand. Dynamic analysers are sound, i.e., they contain no false positives: a program cannot crash on an error that does not exist. Their reports are also much easier to understand, typically containing counterexamples expressed as tests that can be run. The problem is that the tests have to be created, either manually, or by using tools like JCrasher [13], that generate random inputs. Some tools were, therefore, designed by combining the concepts of static analysis and dynamic analysis.

Csallner et al. took their JCrasher tool and added a static analysis component to it by combining it with ESC/Java [19], the annotation-based static analyser discussed earlier, and called the tool Check ' $n$ ' Crash [14]. In Check ' $n$ ' Crash, a program is tested by statically analysing it using ESC/Java. This results in a list of counterexamples, detailing all code fragments it considers to be possible errors. These counterexamples are then used by JCrasher to generate tests that will cause the expected error to occur. These tests are run against the 
program and all tests that have caused an error are, subsequently, reported, while all tests that ran without causing an error, are discarded. This approach improves on JCrasher, by focusing its test generation through counterexamples, while improving the soundness of ESC/Java by confirming whether a counterexample is an error, before reporting it.

The problem with using ESC/Java, is that, in order to use it to its full potential, it still requires annotations to be added to the code. To solve this problem, Csallner et al. added another tool to Check ' $n$ ' Crash, called Daikon [18], and called the resulting tool DSD-Crasher [15]. DSD-Crasher uses Daikon to monitor a program during execution and generalise the observed behaviour of the variables to invariants. These invariants are then used to annotate the code, where Check ' $n$ ' Crash can begin its testing. However, to monitor the program and derive the invariants, Daikon needs tests to run through the entire functional domain of the program.

Here, it becomes evident that by combining dynamic analysis with static analysis, one could benefit from the automated nature of static analysers, while benefiting from the soundness of dynamic analysers. However, an important question to be asked is: At which point is it safe to say that a program has been sufficiently tested? In the following sections, the concept of test adequacy will be explored.

\subsection{Introduction to Test Adequacy and Coverage}

In the previous section, various testing techniques were discussed that covered testing for the purposes of error detection, as well as, proving the absence of possible errors. These testing techniques do not consider the history of the program. Every testing cycle is regarded as a separate entity. However, testers are often required to run regression tests. Regression testing is a form of testing where the program under development is tested, during development, to ensure that all areas that operate correctly, continue to do so, as new features are added. Testers are assisted by regression testing in detecting the presence of bugs sooner and locating the bug faster, since they know what has changed during the period between the previous regression cycle and the current one. Testers, generally, create test cases to automate regression testing.

A test case is defined as an input with which the program being tested is executed during testing. These test cases can be grouped together to form a test suite, or test set [47]. Test sets 
are created to satisfy a test adequacy criterion, often also called a test data adequacy criterion. A test adequacy criterion specifies a testing requirement that, when satisfied, implies that the tested program is without errors.

Zhu et al. present a formal definition of test adequacy criteria and classify them [47]. An explanation of these definitions and classifications is given in the next section, followed by a section on code coverage.

\subsection{Test Adequacy Criteria}

\subsubsection{Introduction}

Test adequacy criteria have two notions associated with them. First, an adequacy criterion is considered to be a stopping rule for itself, determining when the program has been sufficiently tested so that the checking of the criterion may be halted. An adequacy criterion can be formalised as a function $C$ that takes a program $p$, a specification $s$, and a test set $t$ and gives a truth value true or false. Formally, let $P$ be a set of programs, $S$ be a set of specifications, $D$ be a set of inputs of the programs in $P, T$ be a set of test sets, such that $T=2^{D}$, where $2^{X}$ denotes the set of subsets of $X[47]$.

Definition 2.7.1.1 (Test Data Adequacy Criteria as Stopping Rules: [47]) A test data adequacy criterion $C$ is a function

$$
C: P \times S \times T \rightarrow\{\text { true, false }\}
$$

$C(p, s, t)=$ true means that $t$ is adequate for testing program $p$ against specification $s$ according to the criterion $C$, otherwise $t$ is inadequate.

Second, test data adequacy criteria provide measurements of test quality when a degree of adequacy is associated with each test set, so that it is not simply classified as either sufficient or insufficient. For example, the percentage of code coverage is often used as an adequacy measurement. Thus, with an adequacy criterion $C$, formally defined as a function $C$ from a program $p$ with a specification $s$ and a test set $t$, the degree of adequacy $r$ can be defined as $r=C(p, s, t)[47]$. 
Definition 2.7.1.2 (Test Data Adequacy Criteria as Measurements: [47]) A test data adequacy criterion is a function $C$,

$$
C: P \times S \times T \rightarrow[0,1]
$$

$C(p, s, t)=1$ means that the adequacy of testing the program $p$ with the test set $t$ with respect to the specification $s$ is of degree $r$ according to the criterion $C$. The greater the real number $r$, the more adequate the testing.

These two notions of test data adequacy criteria are closely related to one another. If an adequacy criterion states that every statement in a program must be executed at least once, then the criterion $C(p, s, t)$ will only be true if every statement is executed. However, if a test set is considered adequate if only $80 \%$ of the statements are executed, then, with adequacy measurement $M$, and degree of adequacy $r$, the stopping rule $M_{r}$ would be so that a test set is adequate if, and only if, the adequacy degree is greater than, or equal to, $r$, with $r$ at $80 \%$.

$$
M_{r}(p, s, t)=\text { true } \Leftrightarrow M(p, s, t) \geq r
$$

An adequacy criterion is an essential part of any testing method. It plays two fundamental roles. In the first instance, it specifies a particular software testing requirement, and, hence, determines test cases to satisfy the requirement. Second, it determines the observations that should be made during testing. For example, a criterion which specifies that each statement should be executed, will require that each statement executed be observed, while a criterion in which each execution path should be followed, will have no use for the observation of statements executed, but will require that all executed paths be observed and recorded.

\subsubsection{Categories of Test Data Adequacy Criteria}

Zhu et al. [47], identified three categories for the classification of adequacy criteria: Classification by source of information, classification by prospective usage of the software, and classification by the underlying testing approach.

\section{Classification by source of information}

The most common classification is by the source of the information used to specify testing requirements. The adequacy criterion is, therefore, 
- specification-based, which specifies the required testing in terms of the features defined in the functional specifications or requirements of the program, e.g., the spellchecking feature of a word processor. A test set is adequate if all the defined features have been thoroughly tested;

- program-based, which specifies testing requirements in terms of the function of the program under test, e.g., a source code compiler that needs to interpret all allowable syntax of the programming language and convert it to an executable format. A test set is adequate if the function of the program has been thoroughly tested; and

- combined specification-based and program-based criteria, which use the ideas of both specification-based and program-based criteria.

It is important to remember that with specification-based, program-based, and the combined criteria, the correctness of program outputs must be checked against the specification or requirement.

\section{Classification by prospective usage of the software}

Some adequacy criteria may be more concerned with whether the test cases cover the data that are most likely to be frequently used as input during the operation of the software. These criteria are called

- interface-based criteria, which specify testing requirements only in terms of the type and range of software input, without reference to any internal features of the specification or the program.

\section{Classification by the underlying testing approach}

There are three basic approaches to software testing:

- structural testing, which specifies testing requirements in terms of the coverage of a particular set of elements in the structure of the program or specification;

- fault-based testing, which focuses on finding bugs in the software. An adequacy criterion of this approach is some measurement of the fault detecting ability of the test set; 
- error-based testing, which requires test cases to check the program for certain errorprone points, according to our knowledge about how programs typically depart from their specification.

\subsubsection{Focus of this Thesis}

As the title of this thesis suggests, the focus of this study lies in the calculation of code coverage, which, according to the above-listed classifications of test adequacy criteria, falls under structural testing.

\subsection{Code Coverage}

Code coverage consists of adequacy criteria specifying that certain elements of a program be covered. To cover an element of a program means to execute or analyse that element during testing.

Our discussion of coverage is divided into two subsections: specification-based and programbased coverage [47].

\subsubsection{Specification-based Coverage}

Specification-based coverage, often referred to as functional coverage [36, 21, 40], has two possible roles in testing. The first role is to provide information for test case selection when building test sets, to maximise the test adequacy of the set [47]. Its second function is to analyse the adequacy of the test suite when testing the functionality of the program against its specification, similar to black-box testing. This is useful when the program has to conform to some universal specification standard [36] like network protocols, or when verifying a hardware design $[20,2]$. However, because specification-based coverage is specification dependent, finding an existing coverage tool to measure specification-based coverage of a program is difficult, since most tools have the coverage models hard-coded into them [21]. To analyse specification-based coverage, developers have to either try and modify existing tools to support their specification, or create a new coverage tool to test their program specification. To improve on this, tools such as Comet [21] were developed to separate the coverage model definitions from the tool. This allows developers to define their own coverage models [20], without having to build an 
entire new tool from scratch.

Since the formal method used in this thesis is symbolic execution, which is specification independent, specification-based coverage is not used for the purposes of this thesis. The focus of this study is, therefore, program-based coverage.

\subsubsection{Program-based Coverage}

Program-based coverage is split into two groups of criteria: data-flow criteria and control-flow criteria [47].

\section{Data-flow criteria}

Data-flow criteria, as the name suggests, focus on the coverage of data-flow testing in a program. Data-flow testing investigates the usage of data values in a program and how these values affect the execution of the program. The checking of this criteria is similar to model checkers, where model checking analyses program states that represent data values at specific points during program execution. However, data-flow criteria falls outside the scope of this thesis, and interested readers are referred to [47].

\section{Control-flow criteria}

Control-flow criteria focus on the coverage of the control-flow graph of a program. The controlflow graph is a graphic interpretation of the execution of a program, where the nodes represent code blocks, branches occur at conditional statements, and the edges represent the conditions required to hold for that path to be followed.

Every execution of a program corresponds to a path in the control-flow graph, known as the execution path, from the begin node to the end node. The begin node represents the entry point of the program, and the end node represents the point of program termination.

The most common control-flow coverage criteria will be discussed in the following paragraphs.

Statement Coverage Criterion A very basic testing requirement is that every statement in the code has to be executed at least once. This testing requirement is known as the statement coverage criterion. 
Definition 2.8.2.1 (Statement Coverage Criterion: [47]) A set $P$ of execution paths satisfies the statement coverage criterion if and only if for all nodes $n$ in the flow graph, there is at least one path $p$ in $P$ such that node $n$ is on the path $p$.

Even with adequate tests, it is possible to not achieve full statement coverage. This is due to the possibility of infeasible statements, also referred to as dead code. Dead code is code that cannot be executed during program execution, because conditions required for the code to execute containing contradictions, e.g., as in the code snippet displayed in Figure 2.7, p. 36.

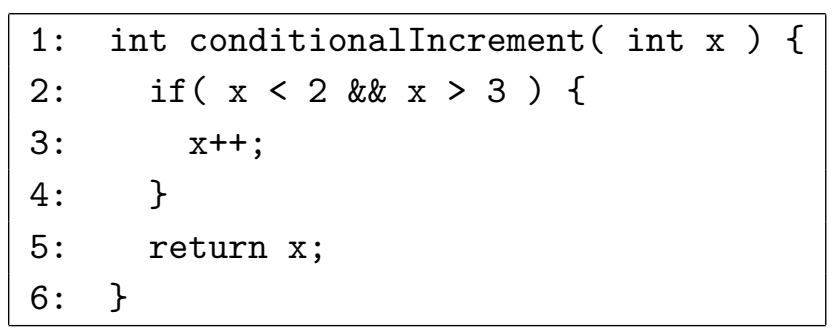

Figure 2.7: This method is a simple example to illustrate infeasible statements. The parameter received is incremented only when the value is less than 2 and more than 3 . These conditions are contradicting, and, therefore, line 3 will never be executed. Line 3 is, therefore, dead code.

It is also important to note that there is a difference between statement coverage and line coverage. A single source code line can contain more than one statement, e.g., a multiconditional if statement, or more complex Java statements. See Figure 2.8, p. 36 for an example the difference between line coverage, and statement coverage [17].

\begin{tabular}{ll|l|}
\hline 1: int $x ;$ & $1:$ int $x=\operatorname{var}>0 ?-1: 1 ;$ \\
$2:$ if $(\operatorname{var}>0)\{$ & \\
$3: \quad \mathrm{x}=-1 ;$ & \\
$4:\}$ else \{ & \\
$5: \quad \mathrm{x}=1 ;$ & \\
$6:\}$ &
\end{tabular}

Figure 2.8: This shows the difference between statement coverage and line coverage. The left side contains an example of a simple conditional assignment. The right side contains the same conditional assignment compressed into a single line of code. Full line coverage on the right does not imply full line coverage on the left, while full statement coverage on either side implies full statement coverage on both sides.

Statement coverage is a weak coverage criterion since some control-flow transfers may be missed. This becomes apparent with control statements such as for loops, while loops, goto 
statements, and similar examples, since any jump back to previously executed statements is irrelevant in statement coverage.

Branch Coverage Criterion Where statement coverage requires statements to be checked, branch coverage requires the checking of control transfers.

Definition 2.8.2.2 (Branch Coverage Criterion: [47]) A set $P$ of execution paths satisfies the branch coverage criterion if, and only if, for all edges e in the flow graph, there is at least one path $p$ in $P$ such that $p$ contains the edge $e$.

Branch coverage is stronger than statement coverage, since full branch coverage also implies full statement coverage. Therefore, a test set that satisfies the branch coverage criterion also satisfies the statement coverage criterion. This relationship between adequacy criteria is called the subsumes relation [47].

Having full branch coverage, however, does not necessarily mean that all possible execution paths have been explored.

Path Coverage Criterion The path coverage criterion is the testing requirement that all combinations of control transfers are checked.

Definition 2.8.2.3 (Path Coverage Criterion: [47]) A set $P$ of execution paths satisfies the path coverage criterion if, and only if, $P$ contains all execution paths from the begin node to the end node in the flow graph.

Path coverage is stronger than branch coverage, because the requirement is that all possible execution paths be explored. Path coverage subsumes branch coverage, and, therefore, statement coverage as well. However, this criterion is too strong to be practically useful for most programs, due to the possibility of an infinite number of different paths in a program with loops [47]. This is the same issue encountered when using symbolic execution to analyse a program with symbolic loops, as discussed under symbolic execution.

Multiple Condition Coverage The previously discussed coverage criteria are all based on the control-flow graph, and do not take the actual program text into account. Condition coverage, on the other hand, focuses on the conditions of control transfers in the program. 
A test set that satisfies the condition coverage criterion contains test cases such that every condition in the program is evaluated to true, and evaluated to false at least once. This criterion is then extended to the multiple condition coverage criterion to allow for multiple conditions available in most high-level programming languages.

Definition 2.8.2.4 (Multiple Condition Coverage Criterion: [47]) A test set $T$ is said to be adequate according to the multiple-condition-coverage criterion if, for every condition $C$, which consists of atomic predicates $\left(p_{1}, p_{2}, \ldots, p_{n}\right)$, and all possible combinations $\left(b_{1}, b_{2}, \ldots, b_{n}\right)$ of their truth values, there is at least one test case in $T$ such that the value of $p_{i}$ equals $b_{i}$, $i=1,2, \ldots, n$.

\section{Issues with Control-flow adequacy criteria}

The issue of unreachable statements, or dead code, was mentioned on the previous page. This issue occurs in all the discussed coverage criteria that measure adequacy from the control-flow graph, i.e., statement coverage, branch coverage, and path coverage. This is because it is impossible to recognise the existence of dead code from the graph, since it models execution behaviour, not program text. Unreachable statements will not be modelled in the control-flow graph, because they are not part of the execution behaviour of the program. Since condition coverage is measured from the program text, it is possible to identify dead code. Later in this study it will become evident how symbolic execution is used to improve on this issue by assisting in the identification of dead code.

Another issue mentioned was that of loops, where, potentially, an infinite number of possible paths may exist in a program with loops. Similar to the issue with symbolic loops in symbolic execution, it is potentially impossible to obtain full path coverage due to the infinite number of test cases that will be required to evaluate the infinite paths. Much research has gone into finding ways to find suitable subsets of execution paths. Some of the examples include: selecting paths that contain no redundant information, selecting paths of length less than or equal to $n$ (called the length- $n$ path coverage criterion), and using loop counting (known as the loop count-K criterion. Loop counting involves limiting the number of iterations a loop can follow to a natural number $K$. The solution for handling symbolic loops during symbolic execution is also a form of the loop count- $K$ criterion. 


\section{Further Reading}

The above discussions on test adequacy and coverage is limited to the scope of this thesis. Interested readers are referred to Zhu et al. [47], as well as Kaner [30], who compiled a list of as many as 110 different types of coverage criteria.

\subsubsection{Measuring Coverage}

There are various approaches to measure the criteria. Atlassian, the creators of Clover [9, 46], identified three approaches to measure code coverage of Java programs:

1. Source code instrumentation is an approach where instrumentation statements, such as annotations or method calls to the coverage calculation tool, are added to the source code. The code is then compiled to produce an instrumented assembly.

2. Intermediate code instrumentation is an approach where the compiled class files are instrumented by adding new byte code, and a new instrumented class is generated.

3. Run-time information collection is an approach that collects information from the runtime environment, as the code executes, to determine coverage information.

As the program under test is executed, coverage is measured according to one, or a combination, of these measuring approaches.

\subsubsection{Coverage Tools}

There are a number of coverage tools available that are designed to measure one or more of the coverage criteria. Among these tools are Clover [9, 46], JCover [26, 46], and EMMA [17, 46].

Clover uses source code instrumentation to instrument Java source code. This is done by integrating Clover into the compilation process to add the instrumentation data to the code before it is compiled. After compilation, the user needs to run the test suite manually to allow for coverage to be measured. Clover measures the coverage by using the statement coverage criterion (Definition 2.8.2.1, p. 36), and the branch coverage criterion (Definition 2.8.2.2, p. 37). It also measures method coverage, which involves checking whether all methods in the source code are executed during testing. 
JCover can use either source code instrumentation, or intermediate code instrumentation, depending on whether it is using source code, or compiled class files. Coverage is then measured similarly to Clover, requiring a build and execution of the test suite. JCover also has a coverage API which allows developers to control JCover, during run-time, through annotations in the code. It focuses mainly on statement coverage, and branch coverage, while also supporting method coverage, class coverage, file coverage, and package coverage. Class coverage involves checking whether every class in the program has been executed, while file coverage checks all non-Java resources such as properties files, $X M L$, and others. Package coverage checks whether all defined packages, i.e., all namespaces within a Java project, are executed during testing.

EMMA uses intermediate code instrumentation, instrumenting the byte code by either compiling the class files with $E M M A$, or by using the custom class loader of EMMA to instrument the class files on-the-fly. Its main focus is basic block coverage, extending it to line coverage, method coverage, and class coverage. Basic block coverage is the coverage measurement of basic code blocks, these being blocks of code without any control statements. Thus, if the first statement in a code block is executed, the last statement in the block will be executed, regardless of variable values, conditional statements, and other elements in the program.

\subsection{Conclusion}

This chapter covered some of the theory and concepts behind testing and test adequacy. Some of the focus points of this thesis were also identified. At this point, the reader should have a basic understanding of the theory behind program analysis and testing, the formal method known as symbolic execution, and the concept of test adequacy and some of the coverage criteria that are available.

In the next chapter, the way in which symbolic execution is used to assist in testing and coverage calculation, as well as how this implementation was integrated into an Eclipse plug-in, will be discussed. 


\section{Chapter 3}

\section{Design and Implementation}

\subsection{Overview}

The previous chapter discussed the theory behind the concepts and techniques that are used in this thesis. This chapter will now discuss how these techniques were applied and combined into an Eclipse plug-in named ATCO.

\section{ATCO}

$A T C O$ is an acronym compiled from Automated Test Coverage Calculation and GeneratiOn. The purpose of $A T C O$ is to study the practical applicability of using symbolic execution to calculate test case coverage, and then use the results from the coverage analysis to automatically generate tests for areas that are not covered. The tool executes in three separate phases, where each phase uses the results from the previous phase. The first phase is the information gathering phase, which runs symbolic execution on the classes under test, and builds the data structures needed in later phases. The second phase is the information analysis phase, where the data structures are analysed while existing JUnit test cases are executed to calculate test coverage. The coverage statistics are then added to the data structures in preparation for the last phase. The last phase, the result analysis phase, analyses the coverage statistics and then automatically generates test cases to cover the areas that are not yet covered by the existing test suite.

Some of the phases contain expensive computations, which will increase execution time as the complexity and scale of the program under analysis grows. Therefore, Java's Concurrency Utilities [41] are utilised to allow these expensive computations to be executed concurrently. 
Later sections will discuss how this is achieved.

ATCO is built on top of another analysis tool, called Artemis.

\section{Artemis}

Artemis, currently being implemented for academic purposes at Stellenbosch University, is an analysis tool that is designed to detect unhandled run-time exceptions from Java byte code. It operates by symbolically executing a program, trying to detect possible unhandled runtime exceptions. When a possible error is located, it runs constraint solving on the symbolic state's path condition to generate method inputs to reach the potentially erroneous state, and generates a JUnit test with these inputs. The test is executed to determine whether it is a real error or a spurious warning. All real errors are reported, while all spurious warnings are ignored. After a possible error is handled by Artemis, analysis continues.

Artemis contains two components of interest for this thesis:

- Its symbolic execution engine, used in $A T C O$ 's information gathering phase. The engine is slightly modified, as will be discussed later.

- Its test case generation engine, used in ATCO's result analysis phase.

\section{Outline}

This chapter discusses the three phases of $A T C O$ 's execution, as well as an overview of how it was integrated into the Eclipse workspace as a plug-in.

\subsection{Information Gathering Phase}

The first phase in ATCO's execution involves analysing all the classes under analysis and constructing the data structure that will be used in subsequent phases. The analysis of the classes is achieved by using Artemis' symbolic execution engine to symbolically execute each class and then storing the symbolic states in a data structure.

\subsubsection{Artemis' Symbolic Execution Engine}

The symbolic execution engine is built on top of Soot, a Java byte code optimisation framework $[42]$. 
Soot is used to convert the Java byte code to Jimple, a typed 3-address intermediate representation, that is suitable for optimisation [42]. Soot converts classes, one method at a time, using transforms. Each method is parsed and a SootMethod class of the method is generated. This class is then passed to the applicable transform to convert the given method into the required representation.

Soot allows for custom transforms to be added to its conversion process. This allows users to either extend the Soot analysis process, or build new analyses on top of Soot. Artemis builds a new analysis process on top of Soot by creating a SymbolicTransformer class, which is plugged into Soot during its initialisation, and used during its execution.

When Soot calls the SymbolicTransformer class, the SootMethod received from Soot is already in Jimple form. This transformed method is then sent to Artemis for symbolic execution. Artemis initialises a single symbolic state and sets up the symbolic execution environment. It then sequentially runs through the method, one instruction at a time, and applies the action of the instruction to the symbolic state. The symbolic state, therefore, maintains an accurate symbolic representation of the program state after each instruction's execution.

When a symbolic branch is reached, the symbolic state at that point is cloned and pushed onto a stack, thereby preserving the state at that branch. This symbolic state, stored on the stack, represents the symbolic branch before any of its paths are executed. The true path of the branch is executed until its last statement. Thereafter, the symbolic state at the top of the stack is removed. The false path of that symbolic branch is then executed until its last statement. The symbolic state at the top of the stack is again removed, and its false path is executed, and so on. It can be seen that symbolic branches are handled as a depth-first traversal through a Binary Tree.

Since symbolic execution creates execution paths for both the true path and false path of a branch, it is possible for the path conditions of some of these paths to contain contradictions. Contradictions in the path conditions of symbolic states mean that those symbolic states are unreachable. To prevent symbolic execution to be performed on these paths that contain contradictions, Artemis performs path pruning whenever it reaches a branching statement. If a path contains contradicting path conditions, that path is stopped, i.e. no longer symbolically executed.

As discussed in Section 2.4.4, p. 22, a symbolic loop is a special case in symbolic execution. 
During symbolic execution, both the true and false paths of all if statements need to be followed. This will result in an infinite loop when a loop has symbolic constraints. Artemis handles this issue by maintaining a loop counter to limit the number of times a symbolic loop may be executed. Therefore, the branching statement representing the loop will have its true and false paths followed $\mathbf{N}$ times, with $\mathbf{N}$ being the value of the loop counter.

\section{Interprocedural analysis}

Artemis analyses each method interprocedurally, confined to a maximum call depth $\left(C D_{M}\right)$. Whenever a method call is encountered in a method, the current call depth $\left(C D_{C}\right)$ is examined. If

$$
\begin{aligned}
& C D_{C}<C D_{M}, \text { invoke method, } C D_{C}=C D_{C}+1 \\
& C D_{C}>=C D_{M}, \text { ignore method }
\end{aligned}
$$

Whenever a symbolic value of the current method is sent to, and modified in, an invoked method, or is assigned a value returned from the invoked method, these changes would not be reflected on that symbolic value during intraprocedural analysis. By taking into account the effect invoked methods have on symbolic values, additional constraints might be added on these symbolic values. The more constraints added on a symbolic value, the narrower the domain of possible values it may have, becomes. The narrower the domain of a symbolic value, the easier it becomes to identify infeasible paths. Figure 3.1, p. 45 illustrates this.

\section{Modification}

To use the symbolic states for coverage analysis, it is required to be aware of the symbolic states at symbolic branches. When a symbolic branch is encountered, two options are available; either run the relevant test cases testing that method, or build a data structure of all symbolic branches, and then run the test cases. If the tests are run whenever a branch is encountered, each test will have to be executed exponentially many times, as a method may have multiple paths, and each path may have multiple branches. Storing the symbolic states in a data structure will require that each test case is only executed once.

It was mentioned earlier that Artemis maintains a single symbolic state throughout the symbolic execution of the method. When a symbolic branch is reached, the symbolic state is cloned. However, the cloned copy of the symbolic state made at a branch is only used as a 


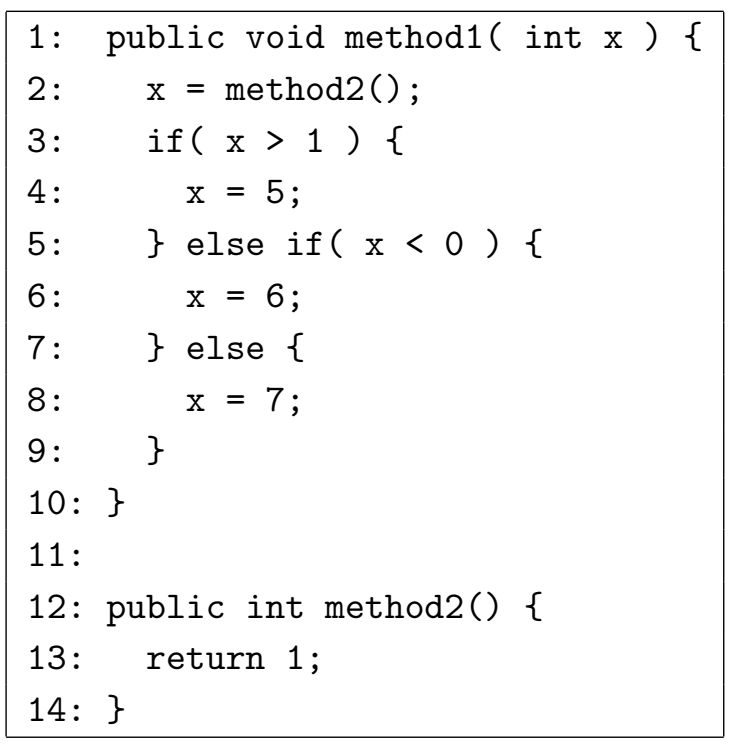

Figure 3.1: Interprocedural infeasible path identification is illustrated in the above code snippet. If method 1 were to be symbolically executed intraprocedurally, the invocation of method 2 would be ignored, and all three paths through method 1 would be considered possible. With interprocedural analysis, method 2 would be invoked, and it would be discovered that only the path at line 8 would be executable. The other two paths (line 4 and line 6 ) would be identified as unreachable. Line 4 and line 6 are, therefore, both infeasible paths.

snapshot of the symbolic state at that point. To use symbolic states in $A T C O$, the symbolic state for each statement in the program needs to be stored separately. Knowing the relationship between the different symbolic states is also important, i.e., knowing which symbolic states form which execution paths.

A symbolic execution tree $(S E T)$, as discussed in Section 2.4.3, p. 21, is used to store the symbolic states of a method. All SETs constructed during symbolic execution is grouped by class, allowing $A T C O$ to access each $S E T$ of each class during coverage calculation.

\subsubsection{Symbolic Execution Tree}

\section{Structure}

The SET is a Binary Tree, as each branching node may have, at most, two children, i.e., a true path and a false path leading from it. Each node in the SET contains the entire symbolic state at that point in the method. The structure of the SET represents the flow of execution through the method, a root node for the first statement of the method, a branch in the tree for every corresponding branching statement in the method, and a leaf node for the 
last statement of every possible path in the method. The unique paths between the root and leaf nodes indicate all the possible execution paths through the method, except when symbolic loops are present.

When a method contains a symbolic loop, the unique paths between the root and leaf nodes do not necessarily represent all possible execution paths through the method. This is because loop iterations are limited during symbolic execution. In such a situation, the SET would only contain a subset of the execution paths. The size of the subset depends on the limit set on loop iterations.

\section{Special constructs}

Most of the control-flow statements in Java are represented as if statements in byte code, after compilation. So a SET representation of a method may easily be derived. However, the switch statement in Java is handled differently. The switch is represented as a special tableswitch or lookupswitch construct, to allow the $J V M$ to handle the switch statement more efficiently. This results in a single statement that may now have more than two paths extending from it. To enable $A T C O$ to handle this special construct, an additional transformation is required to represent this construct in the $S E T$, as each node in the SET may have, at most, two children. This additional transformation involves inserting blank nodes into the $S E T$, which may serve as branching points for the different branching cases of the switch statement. Figure 3.2, p. 46, illustrates this solution with an example.

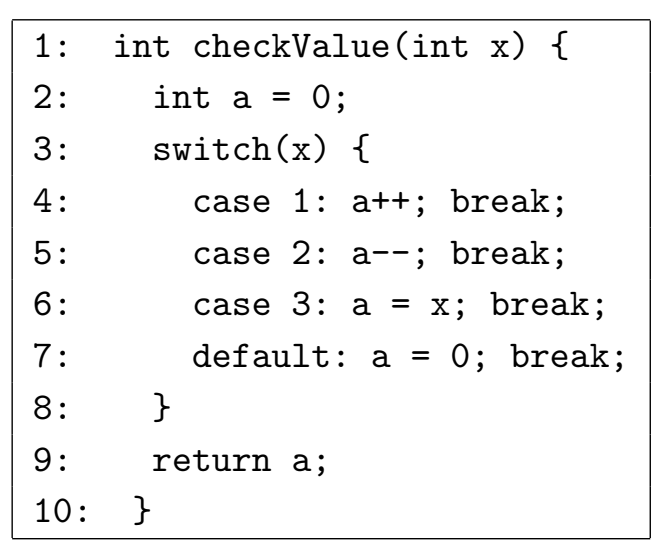

Figure 3.2: This figure displays a simple code example of a Java switch statement. The variable $a$ is modified in a certain manner, depending on the value of $x$ received as input parameter. Figure 3.3, p. 47 displays the resulting SET, constructed from symbolically executing this method. 


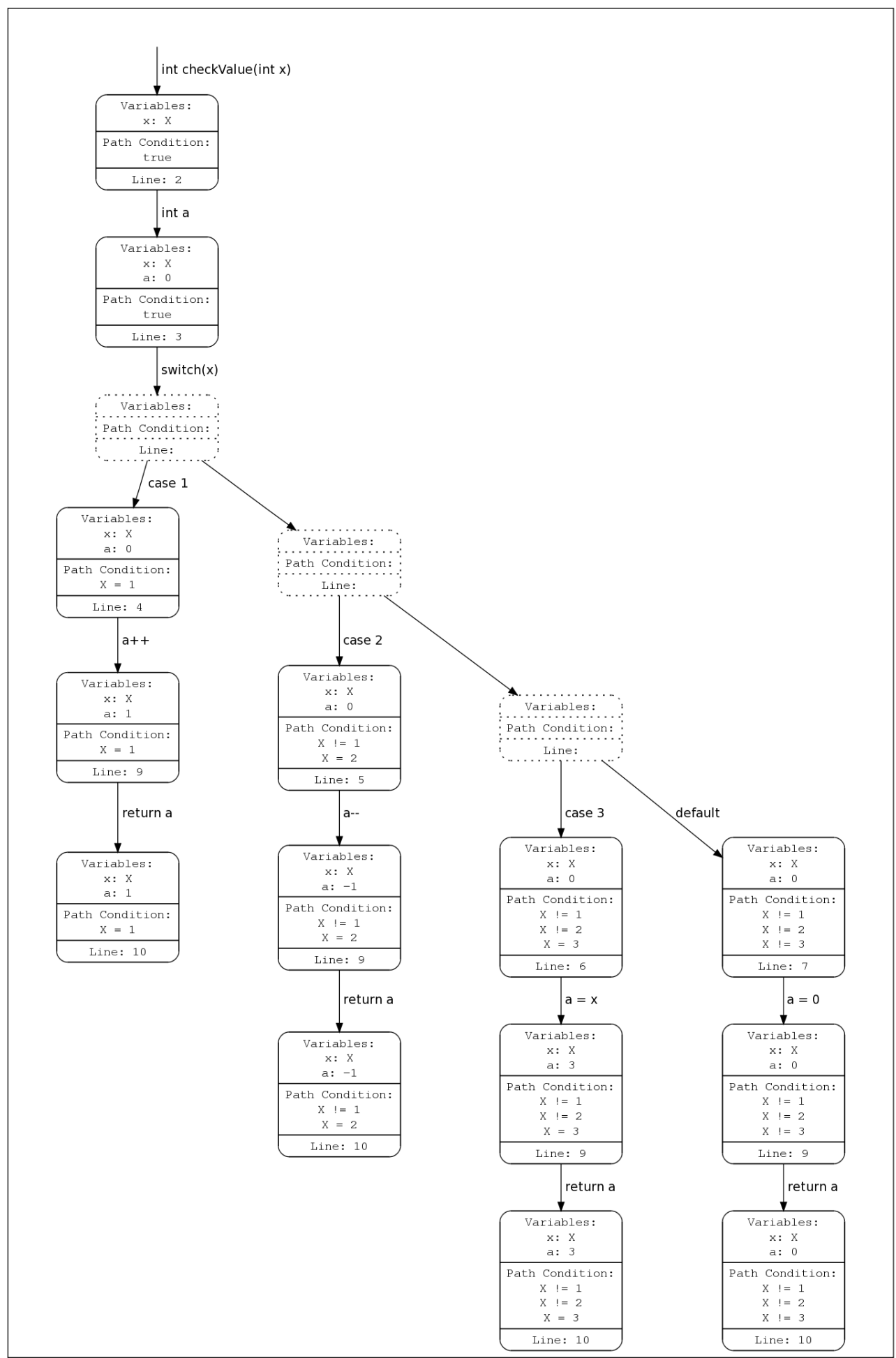

Figure 3.3: This is a SET of the switch statement in the code example, displayed in Figure 3.2 , p. 46 . The nodes contain the line number to be executed, and the edges contain the statement that was executed. The dotted nodes indicate the blank symbolic states, inserted into the $S E T$, which serve as the branching points for the different cases. 
Interprocedural analysis introduces a similar scenario to that of the switch statement. If a method, invoked during interprocedural analysis, contains symbolic branches, and returns a value to the method from which it was invoked, that invocation statement may have two, or more, paths extending from it. To maintain an accurate representation of the method under analysis, the SET needs to reflect these paths. This scenario is handled similarly to the solution for handling the switch statement. Blank nodes are inserted into the SET to serve as the branching points for the various paths that may be followed in the method that was invoked. Figure 3.4, p. 48, illustrates this solution, with regards to interprocedural analysis, with an example.

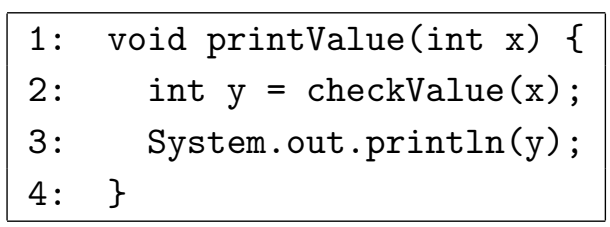

Figure 3.4: This figure displays a simple code example, used to illustrate the branching that may occur during interprocedural analysis. With the checkValue method example, displayed in Figure 3.2, p. 46, this example contains a method that invokes checkValue and prints the resulting value. The resulting SET is displayed in Figure 3.5, p. 49.

\section{Why use a SET?}

Because of the nature of symbolic execution, it becomes difficult to effectively and accurately group statements into repeating code blocks. The best example for illustration is symbolic loops. The same code block gets executed with each iteration of the loop. However, with symbolic execution, the path condition, and, in fact, the symbolic state of the program, is different for each iteration. Simply grouping statements into code blocks would therefore not yield an accurate representation of the symbolic states achievable by that block. By storing these symbolic states separately, the SET structure simplifies the process of storing and differentiating between these types of code blocks. To indicate this, recall the symbolic loop example displayed in Figure 2.6, p. 25. With a loop counter of three, i.e., the loop will be iterated three times, Figure 3.6, p. 50, displays the resulting $S E T$ if the statements were grouped into repeating code blocks. The size of the SET is smaller than before, but all the data regarding every iteration of the loop is lost, apart from the last iteration.

During symbolic execution, constraint solving may be used to prune infeasible paths, also 


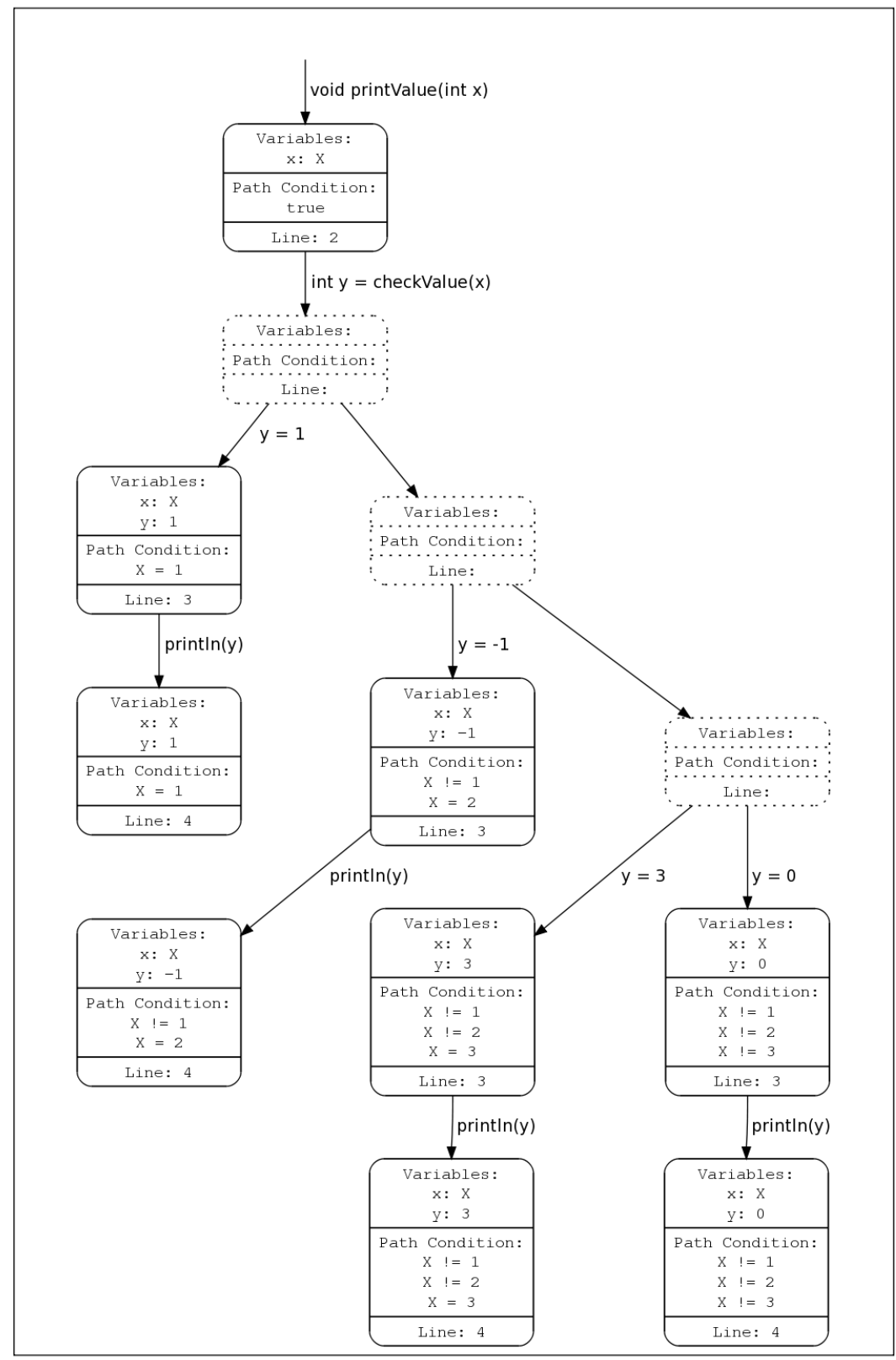

Figure 3.5: An example $S E T$ to illustrate the branching that may occur during interprocedural analysis, with the code example displayed in Figure 3.4, p. 48. The checkValue method returns one of four values, namely $1,-1,3$, or 0 , depending on the value of $x$ received. The nodes contain the line number to be executed, and the edges contain the statement that was executed. The dotted nodes indicate the blank symbolic states, inserted into the $S E T$, which serve as the branching points for the different values that are returned by checkValue. 


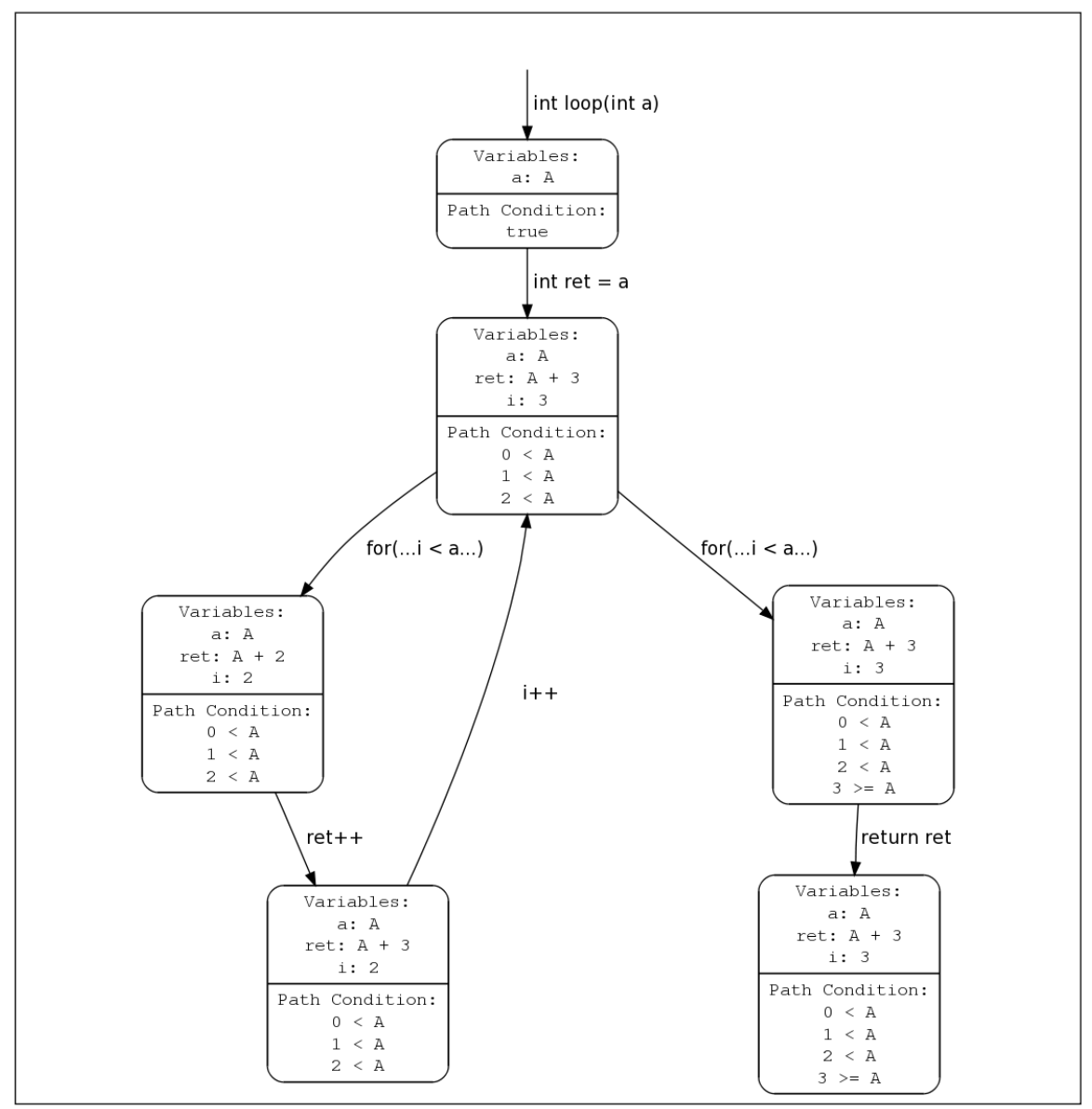

Figure 3.6: This $S E T$ is the result of the code in Figure 2.5, p. 24, being symbolically executed, and stored in a $S E T$ when statements are grouped into repeating code blocks. All the data regarding every iteration of the loop is lost, apart from the last iteration. The edges contain the statement that was executed.

called dead code or unreachable statements, from the analysis. Artemis uses a constraint solver, called Choco [38], to solve path conditions for testing and path pruning. Constraint solving involves determining whether there is a solution to a given set of constraints on variables, as represented in a constraint model. Thus, infeasible paths may be identified by creating a constraint model from the path condition of the symbolic state and attempting to solve the model with Choco. When a path condition contains a contradiction on the constraints of a symbolic value, that set of constraints, or rather the path condition, is unsatisfiable. An unsatisfiable path condition indicates that its symbolic state cannot be reached, i.e., it is a dead state. Therefore, the code represented by that symbolic state is dead code. Constraint solving is used similarly during coverage analysis in ATCO. Constraint solving is an expensive 
computation, so minimising the number of times constraint solving is run may greatly reduce the time required to perform coverage analysis. Storing the states in a $S E T$ minimises the amount of constraint solving required to calculate coverage by limiting the computation to only the first non-branching nodes after a branching node, as will be discussed in Section 3.3.

\section{Types of coverage derivable from the SET}

Section 3.3.3, p. 55, will discuss how $A T C O$ measures branch coverage (as defined in Definition 2.8.2.2, p. 37), multi-conditional coverage (as defined in Definition 2.8.2.4, p. 38), and statement coverage (as defined in Definition 2.8.2.1, p. 36). However, there are a multitude of other coverage criteria that may be derived using the SET after branch coverage, multi-conditional coverage, and statement coverage have been calculated, and indicated, on the SET. A list, taken from [30], is displayed below. This list contains, but is not limited to, a number of coverage criteria that are not directly implemented within $A T C O$, but are inadvertently measured in, or can be derived from, the $S E T$, together with the symbolic states within.

Line coverage: $A T C O$ already uses line numbers in the statement coverage calculation process. Line coverage can, therefore, easily be derived from statement coverage.

Path coverage: Each SET represents the execution paths that may be followed within the method it represents. When no symbolic loops exist, the SET contains all execution paths in the method. Therefore, with full branch coverage in a method, path coverage for that method is subsumed by the branch coverage. However, when symbolic loops are present, a loop counter is used. This limits the execution paths to the subset of paths that have been symbolically executed before the counter limit was reached. Path coverage on the method containing symbolic loops may, therefore, only be derived to the subset of paths symbolically executed. Full path coverage, i.e., path coverage over the entire program's execution, however, cannot be derived with ATCO's current implementation of the SET. Containing all execution paths in each method is not sufficient to derive full path coverage. Every sequence in which each execution path in each method is executed needs to be considered to measure full path coverage. Although it should, theoretically, be possible to collect sufficient data to measure full path coverage, the number of possible paths is still too large to make this criterion practical. 
Loop coverage: With the use of loop counting, set to $n$, each symbolic loop will be symbolically executed $n$ times. Loop coverage can therefore be measured by setting $n>1$. ATCO is able to measure loop coverage by counting the iterations of a loop during test execution. Due to the design of $A T C O$, measuring loop coverage is not performed when redundancy detection is disabled. This is because no further attempts are made to determine whether a loop is hit, if it has already been hit at least once.

Assertion coverage: In the byte code, an assert statement is compiled to a branching statement. To calculate assertion coverage, all assert branches need to be identified. Knowing this, it is possible to calculate the number of assert branches that are covered. However, to identify whether a branch is an assert branch, the original Java source code statements are required. ATCO contains sufficient data in the SET to calculate this coverage criterion, however, due to third-party library constraints, the actual Java source code statements are unavailable. Thus, measuring this coverage criterion is currently not possible.

Method and Class coverage: The SETs represent methods which are grouped together and categorised according to class. Method coverage can, therefore, easily be determined by examining the coverage statistics of that method's SET. If any node in the $S E T$ was hit during testing, the method has been hit at least once. Class coverage requires that at least one $S E T$ for that class was hit during testing.

Component, tool, subsystem, and other Kaner [30] mentions that measuring this coverage criterion is difficult, because programs often rely on off-the-shelf components to which the source code is not always available. However, because coverage is measured from byte code and not source code, it is possible to extend ATCO to measure coverage over all components, open source or closed source. This is because all libraries are, at least, in byte code format to allow them to be used.

Because of the structure and information stored within the SET, it is possible to extend the coverage calculation within $A T C O$ to measure most control-flow coverage criteria. The symbolic states within the $S E T$ contain all the information required to measure many dataflow coverage criteria as well, such as the variable definition-and-use criteria discussed in [47]. However, this does not fall within the scope of this thesis, and was, therefore, not investigated. 


\subsection{Information Analysis Phase}

After the information gathering phase, $A T C O$ has access to the SETs of each method in each class being analysed. This information may be used to calculate the coverage of the existing JUnit test suite over these classes.

Section 2.8.3, p. 39, mentioned three approaches to measuring coverage. The chosen approach for this thesis is the run-time information collection approach. This is done with the use of execution tracing. An execution tracer is attached to the test environment wherein a JUnit test will be run. As mentioned earlier, this phase is executed concurrently, which requires special consideration to be taken during the calculation process.

\subsubsection{Execution Tracing}

Execution tracing is achieved by utilising the Java Platform Debugger Architecture (JPDA) [4]. The JPDA is a debugging architecture used for debugger development, providing the services the Java Virtual Machine (JVM) must provide for tools attempting to perform profiling, debugging, monitoring, thread analysis, coverage analysis, and so on. The highest level layer of $J P D A$ is the Java Debug Interface $(J D I)$. The $J D I$ provides explicit control over the $J V M$, allows for inspection of the JVM's state at any point, event notification such as exceptions being thrown, class loading, method calling, and more, as well as providing the use of breakpoints, watchpoints, and so on. The events are among the services provided by the JDI that was used to perform execution tracing.

Events are triggered at the occurrence of specific operations in the attached JVM. Three events of particular interest for this coverage implementation are: the ClassPrepareEvent, the MethodEntryEvent, and the BreakpointEvent.

Whenever a class is loaded in the $J V M$, the ClassPrepareEvent is triggered before the class is executed. This event is triggered for all classes being loaded, from the core Java classes, to custom libraries, to the class under analysis. This presents many avenues of operation for tools using the JDI. However, for this coverage analysis implementation, only the class under analysis is inspected. When this event occurs for the class under analysis, the class's $S E T$ is traversed to determine at which lines the breakpoints should be added.

Breakpoints are triggers that may be attached to locations in the source code that, when 
reached, will cause a BreakpointEvent before executing the code at that location. Breakpoints will be used to indicate the presence of branches in the execution of methods.

A MethodEntryEvent is triggered when a method is to be invoked. This event allows an analysis tool to investigate the data used in the method's invocation. The method parameters are among the data accessible within this event, which is important to ATCO's coverage calculation process.

\section{Why use Execution Tracing?}

Execution tracing is not the only method that may be used to evaluate which states are hit during test runs. Another method of calculating coverage is to parse the JUnit tests to locate all method calls to the method under test, and run constraint solving over the input parameters and the states in the SET. However, tests may potentially have complex setup methods and require external libraries. Parsing the test and storing all the necessary data to ensure the conditions of the test are an exact representation of its actual execution may be difficult. This may require an expert knowledge of the JUnit test framework. Also, constraint solving would have to be performed across all possible branches in the $S E T$, to determine which path is followed. This could greatly reduce performance as constraint solving is an expensive computation and a method may theoretically contain infinitely many symbolic branches.

With execution tracing, the JUnit tests are executed, resulting in all the environmental data required for coverage calculation to be available via the JDI without any additional computations or knowledge of the JUnit test framework. The environmental data, such as method invocation parameters, or which branch is currently being executed, may be used to greatly reduce any unnecessary constraint solving, as will be seen in later sections.

Execution tracing also automatically extends the coverage calculation to be interprocedural. A JUnit test will typically not directly call all methods in the class it is testing. It will, typically, invoke a certain piece of functionality through a number of method calls. This will, in turn, perform its purpose through other method calls, often private or protected methods, which cannot be invoked directly from the JUnit test. Execution tracing will automatically handle these methods the same as methods invoked directly from the test, which allows for coverage to be calculated on non-public methods, without any additional processing. 


\subsubsection{Test Environment}

The test environment is a $J V M$, created through the $J D I$, in which the JUnit test will be executed. When executing multiple tests, it is important to maintain the integrity of each test. One test run may affect subsequent test runs, thereby compromising the integrity of all tests executed within the same environment. Each test is, therefore, executed within its own test environment. This ensures that the integrity of the test is maintained, while also providing an easily identifiable unit of work to be executed concurrently.

\subsubsection{Calculating Coverage}

$A T C O$ calculates branch coverage, multi-conditional coverage, and statement coverage as defined in Definitions 2.8.2.2 (p. 37), 2.8.2.4 (p. 38), and 2.8.2.1 (p. 36), respectively.

Branch coverage is measured on a Jimple level. So, a branch covered in Jimple does not necessarily translate to the conditional statement being completely covered in Java. This occurs when the conditional statement in Java has multiple conditions. A multi-conditional branching statement in Java is compiled to a series of subsequent single-conditional branching statements in Jimple. The structure of the SET, together with the data stored within the symbolic states, allows for all Jimple statements to be linked to their corresponding Java statements. This allows multi-conditional coverage to be calculated by linking all compiled single-conditional branching statements in Jimple to their corresponding multi-conditional branching statement in Java. The SET also allows for statement coverage to be calculated on a Jimple level from branch coverage. Therefore, with the help of the SET, branch coverage on a Jimple level can be translated to statement coverage, branch coverage, and multi-conditional coverage in Java source code.

\section{Calculation during execution tracing}

The test environment is set up by creating the $J V M$ in which the test will be executed, and passing the $J V M$ the SETs of the class under test at the JDI level. The test execution is then started.

Configuration of the $J V M$ occurs on-the-fly during test execution, when a ClassPrepareEvent is triggered for a class under analysis, i.e., when the class is about to be loaded during execution. The configuration involves adding breakpoints to certain symbolic states in the class's $S E T \mathrm{~s}$, 
before the class is loaded. To add a breakpoint to a symbolic state means to add a breakpoint to the Java source code line number, that the symbolic state represents. Breakpoints are added to the first non-branching symbolic state following a branching symbolic state in the SET, along both the branching symbolic state's true and false paths. This is to cater for the possibility that a branching symbolic state may be immediately followed by another branching symbolic state. The reason for attaching breakpoints to these symbolic states, and not the branching symbolic states themselves, is because breakpoints on branching symbolic states will require two constraint solving operations to determine which path was taken. Having the breakpoints on the first non-branching symbolic state will require no constraint solving, since the path containing the breakpoint is followed during test execution.

Figure 3.7, p. 56 illustrates this configuration with an example.

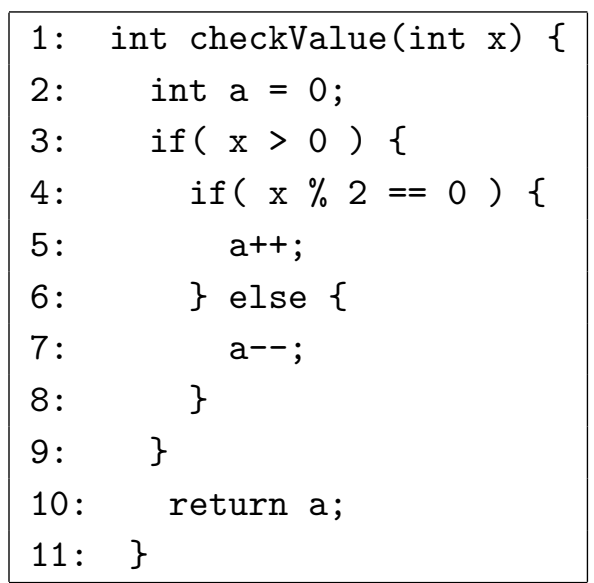

Figure 3.7: This method is a simple example, used to illustrate the breakpoint configuration during coverage calculation. The method returns 0 if $x<=0$, returns 1 if $x$ is positive and even, and returns -1 if $x$ is positive and odd. The resulting $S E T$ is shown in Figure 3.8, p. 57 . The first non-branching symbolic states following branching symbolic states are indicated with dotted nodes.

After the breakpoint configuration, the class is loaded, and test execution continues until a MethodEntryEvent is triggered. When the event is triggered, the parameters are stored locally for the duration of that method's execution. Data related to the parameters include the parameter names, types, and values. Also, during this event, there is a check whether the SET contains any branches. Please be reminded that branches in the SET only occur at branching statements with symbolic conditions. If a method contains no symbolic branches, then that method will have no breakpoints set. In such a case, all statements within the method being 


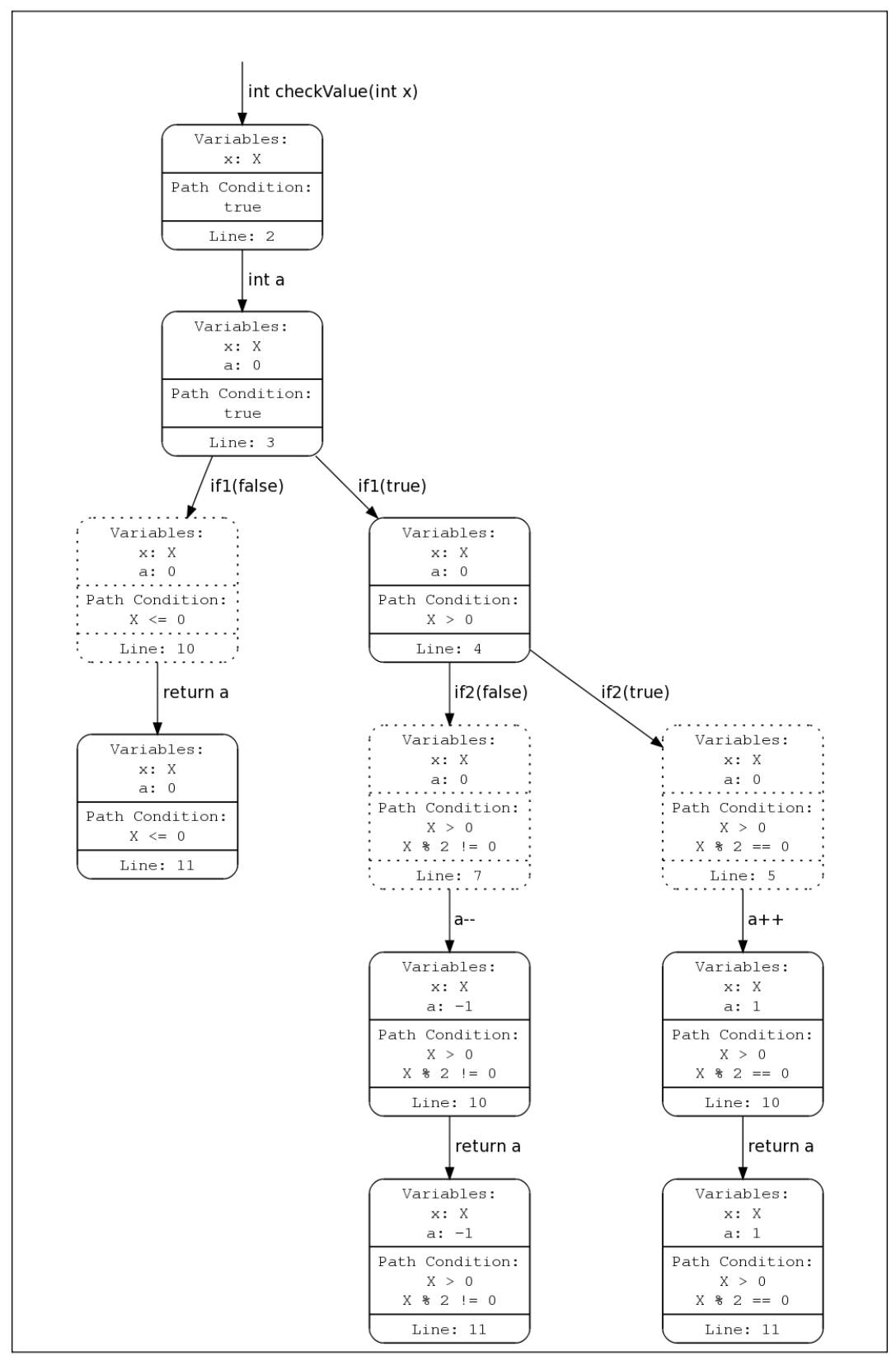

Figure 3.8: This $S E T$ is the result of the code in Figure 3.7, p. 56, being symbolically executed. The nodes contain the line number to be executed, and the edges contain the statement that was executed. The edge if 1 indicates the if statement at line 3 , while if 2 indicates the if statement at line 4 . The values (true) and ( $\mathrm{false}$ ), associated with those if edges, indicate whether the true or false paths were followed with that edge. The dotted nodes indicate the symbolic states to which breakpoints will be added. 
invoked may immediately be marked as covered, as all statements will be executed. When a symbolic state in the SET is marked as covered, a coverage counter, maintained within that symbolic state, is incremented. This counter indicates the number of times the symbolic state was hit during coverage calculation; a hit means the symbolic state was reached during test execution.

Execution continues until a BreakpointEvent is triggered. As previously mentioned, breakpoints are added to the first non-branching symbolic state following a branching symbolic state in the SET. Therefore, when a BreakpointEvent is triggered, a symbolic branch was reached and one of its branches is already followed. If the symbolic branch executed has no symbolic branches preceding it, nested branching excluded, then the statement at the breakpoint will have only one symbolic state representing it. The single symbolic state is, therefore, the symbolic state hit during execution, and this symbolic state's counter is then incremented, with no constraint solving required. If the branch has symbolic branches preceding it, then the statement at the breakpoint will have multiple symbolic states representing it. Each symbolic state, representing the single statement, will have a unique path condition, depending on which paths were followed in earlier branches. An example is displayed in Figure 3.9, p. 59. When a statement has multiple symbolic states representing it, a copy of each symbolic state is made to maintain the integrity of the original symbolic state. Each copy has the method parameters added to the path condition. Each copy, with the method parameters added to the path condition, is then run through the constraint solver to discover which of the symbolic states were actually hit. The symbolic state with a satisfiable path condition is the symbolic state hit at the breakpoint. An exception to this occurs when symbolic loops are involved, which will be discussed later in this section, p. 62.

The $J V M$ continues to run the test and service the triggered events until the test is complete. Upon completion, the SET will have the updated coverage counter values of all the symbolic states that had breakpoints set at their location. A 100\% statement covered method will have the coverage counter of every first non-branching symbolic state following a branching symbolic state in its $S E T$ set to at least 1. For methods with no symbolic branches, i.e., no branching statements in its $S E T$, only the first symbolic state in the $S E T$ will have its coverage counter updated. 


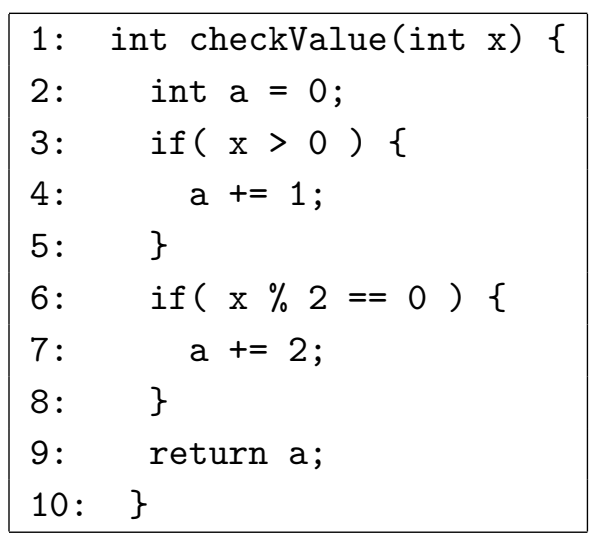

Figure 3.9: This method is a simple example used to illustrate the breakpoint configuration during coverage calculation when a line of code is represented by multiple states. The method returns 0 if $x<=0$, returns 1 if $x$ is positive and odd, returns 2 if $x$ is negative and even, and returns 3 if $x$ is positive and even. The resulting SET is shown in Figure 3.10, p. 60. The first non-branching states following branching states are indicated with dotted nodes.

\section{Updating coverage counters in the $S E T$}

The coverage results, after test execution, show only which paths were followed at each branch in the SET by way of the first non-branching state containing a coverage counter indicating how many times it was hit. Since the $S E T$ is an execution tree with no paths leading to nodes other than a specific node's direct parent or children, these coverage results may be used to accurately calculate which paths in the SET were followed during execution. This is achieved by updating the coverage counter of all the states in the SET. The counters are updated by traversing the $S E T$ depth-first, until the last covered state in an execution path is found, i.e., a state marked as hit, with its direct parent being a branching state, and no branching states succeeding it. Figure 3.11, p. 61, indicates this by showing the selected states as dotted nodes. The coverage counter for each state in that execution path is then updated as follows: With the root state, $S_{r}$, the last covered state, $S_{m}$, the last state in the path of $S_{m}, S_{l}$, their coverage counters $S_{r}^{c}, S_{m}^{c}$, and $S_{l}^{c}$, respectively, and $i=r_{. .}(m-1)$, all states along the path of $S_{m}$ are updated as:

$$
\begin{aligned}
S_{i}^{c} & =S_{i}^{c}+S_{m}^{c} \\
S_{m . . l}^{c} & =S_{m}^{c}
\end{aligned}
$$

This is illustrated in the example displayed in Figure 3.11, p. 61. 


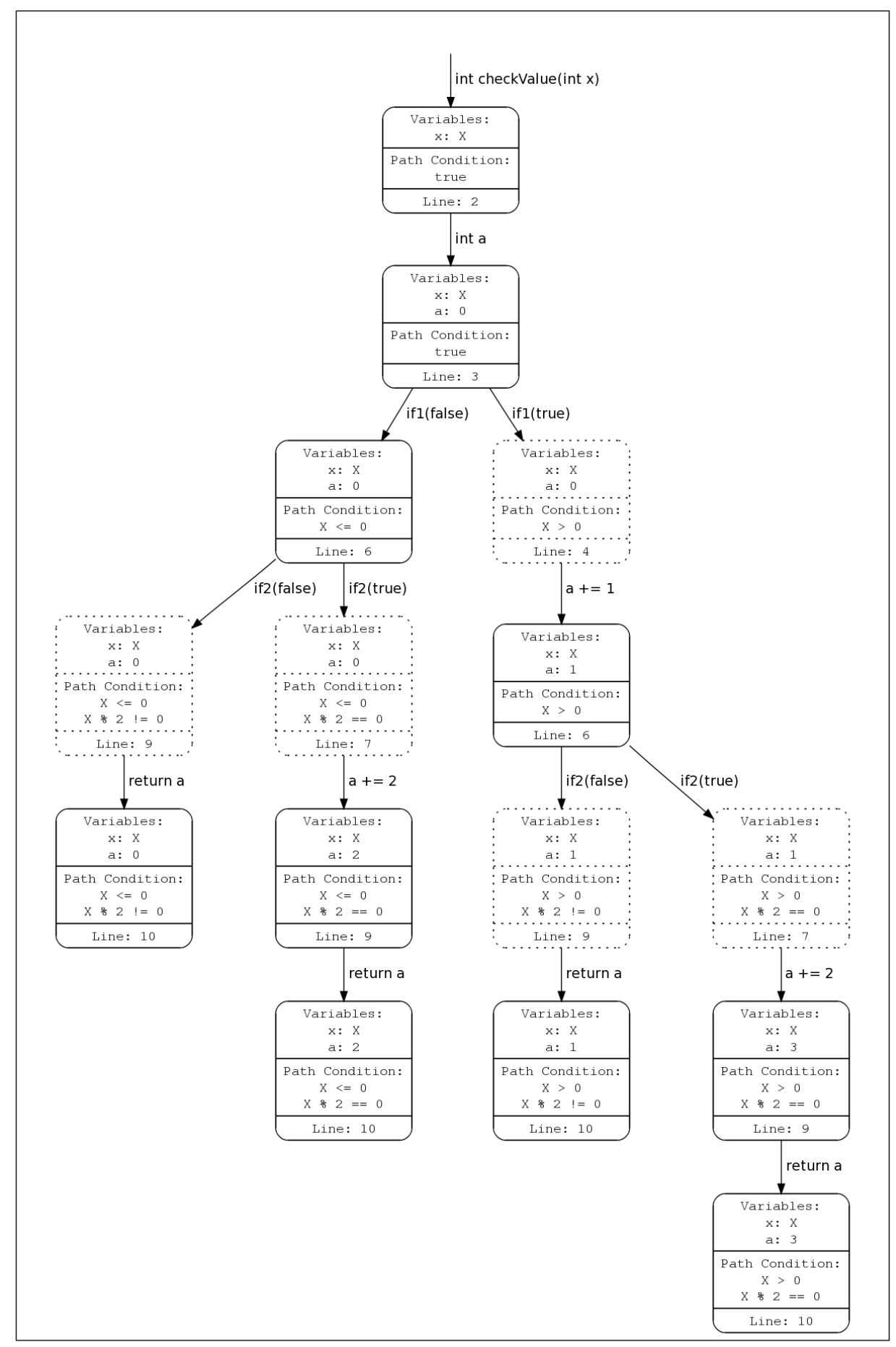

Figure 3.10: This $S E T$ is the result of the code in Figure 3.9, p. 59, being symbolically executed. The nodes contain the line number to be executed, and the edges contain the statement that was executed. The edge if 1 indicates the if statement at line 3, while if 2 indicates the if statement at line 6. The values (true) and (false), associated with those if edges, indicate whether the true or false paths were followed with that edge. The dotted nodes indicate the states to which breakpoints will be added. Note that there are two symbolic states with breakpoints representing lines 7 and 9 in the $S E T$. 


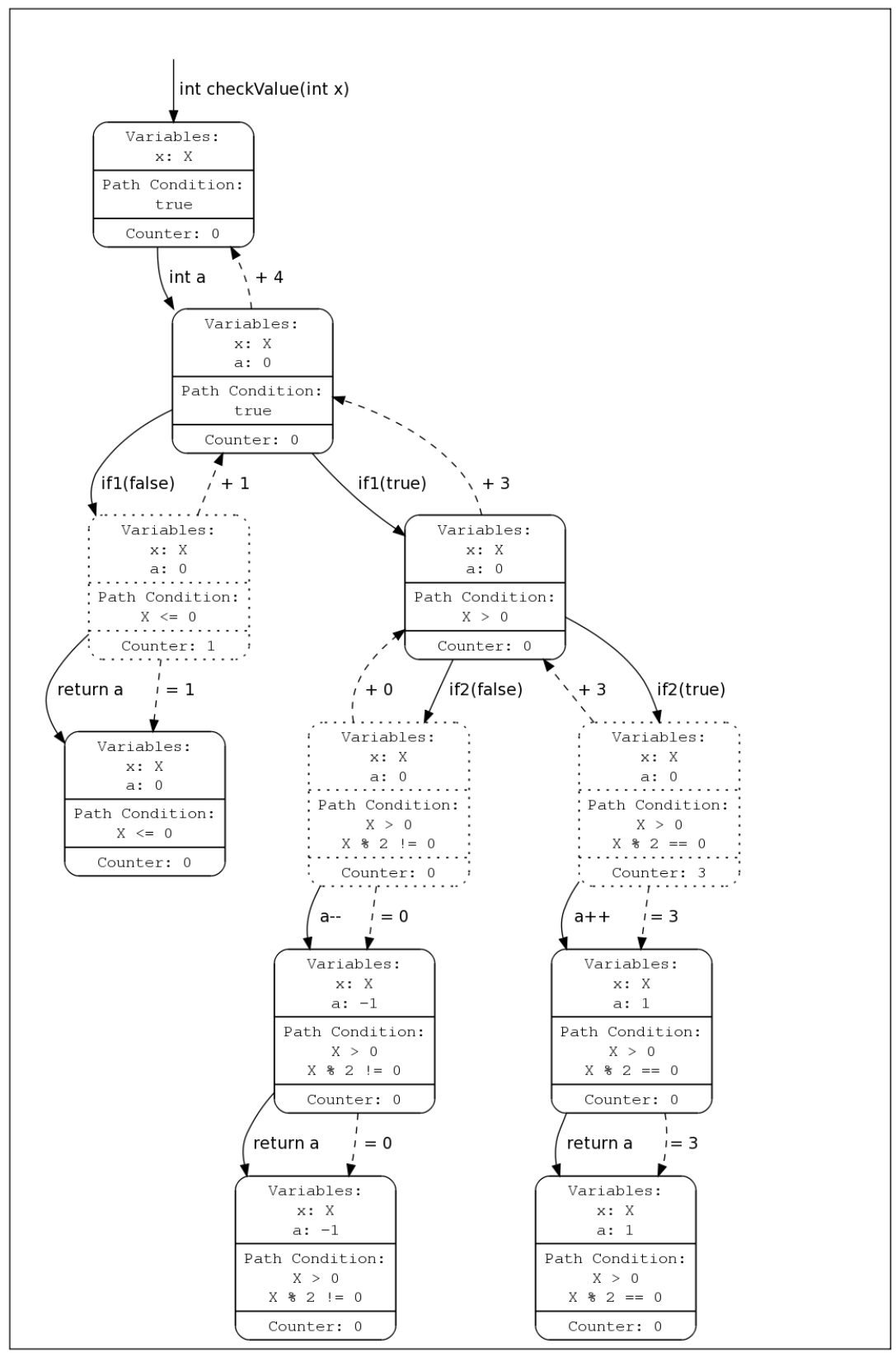

Figure 3.11: This $S E T$ is the result of the code in Figure 3.7, p. 56, being symbolically executed, and a test then being executed. The nodes now contain the coverage counters (Counter) of the symbolic states after execution tracing has been completed, with the dotted nodes being the symbolic states where breakpoints were added. The solid edges, as before, contain the statements that were executed, while the dotted edges illustrate how the coverage counters of the other states are updated. The edge if 1 indicates the if statement at line 3 , while if 2 indicates the if statement at line 4. The values (true) and (false), associated with those if edges, indicate whether the true or false paths were followed with that edge. 


\section{The end result}

After execution tracing is complete and the coverage counters in the SET are updated, the coverage counter of each statement and branch in the $S E T$ will have been updated. This indicates how many times each symbolic state in the SET was hit during testing. Therefore, the result is the branch coverage and statement coverage of the SET on a Jimple level. This information is then used to map the coverage statistics in the SET to the Java level, which will then indicate the branch coverage, multi-conditional coverage, and statement coverage of the SET on a Java level.

\section{Interesting case with symbolic loops}

There is an interesting occurrence when dealing with symbolic loops. With a symbolic value $V$, when a loop condition contains a symbolic condition, e.g., $i<V$, both the true and false paths are followed for the number of iterations as indicated with the loop counter, e.g., $n$. Thus, all the statements in the loop will have $n$ states, e.g., $S_{1 . . n}$, representing them. Figure 3.12, p. 62, displays a code example for a symbolic loop, together with the path conditions of the symbolic states representing the paths.

\begin{tabular}{|c|cc|}
\hline Code snippet & \multicolumn{1}{c|}{ Path conditions } \\
\hline for ( int i $=0 ;$ i $<$, i++ ) \{ & $S_{1}: 0<V$ \\
System.out.println( "Looped" ); & $S_{2}: 0<V, 1<V$ \\
\} & $S_{n}: 0<V, 1<V, \ldots, n-1<V$ \\
\hline
\end{tabular}

Figure 3.12: This table illustrates an interesting case with symbolic loops when calculating coverage using symbolic execution.

The first non-branching statement within the loop will have a breakpoint assigned to it. When this breakpoint is reached with each iteration of the loop, constraint solving will be used to determine which of the $n$ states were hit. The input parameters will indicate that, e.g., $V=v$. If $v>n$, then states $S_{1 . . n}$ will be marked as hit, while $S_{n+1 . . v}$ will not. This is because the number of times the loop is traversed during normal testing is more than the number of times it is traversed during symbolic execution. If $v<n$, then states $S_{1 . . v}$ will be marked as hit, while $S_{v+1 . . n}$ will not. If $v=n$, then all states will be marked as hit. This interesting case will not result in states being marked as covered when they were not hit during testing, but it will result in the number of times they were hit being incorrect. 
To resolve this inaccuracy, the following needs to be considered. During the execution of a method, the symbolic values remain unchanged. The method's execution changes concrete values, path conditions of symbolic states, and the local variables that contain the computational expressions of the symbolic values. Recall Figure 2.1, p. 19. With every iteration of a symbolic loop, the symbolic values will be the same. This results in the same subset of symbolic states having satisfiable path conditions with every iteration. Thus, all symbolic states in the SET, that are part of the subset of symbolic states in the loop that will be marked as covered, are marked as hit with every iteration. So to resolve this inaccuracy, the coverage counters should only be updated with the first iteration of the loop. All subsequent iterations may be followed and monitored, but no coverage counters should be updated for the affected subset. However, due to time constraints during the implementation of $A T C O$, this was not implemented.

\subsubsection{Test Redundancy Detection}

Having a satisfactory level of code coverage indicates that a program is sufficiently tested, but it does not indicate that a program is tested efficiently. Ideally, a test suite should contain no redundant tests. A test is considered redundant if it does not exercise any new behaviour of a program that has not already been exercised by other tests in the suite [45]. These redundant tests increase the resources required to generate, execute, and maintain a test suite, without adding any value to the suite itself.

ATCO provides a mechanism for identifying such redundant tests by gathering statistics on what value each test case adds to the test suite during coverage calculation. Whenever coverage calculation determines which state in the $S E T$ was hit during testing, the statistics of the test that was executed is examined. If the state, hit during testing, has not yet been hit by a previous test, a counter is incremented to indicate that this test has hit a new state, i.e., it is testing a new behaviour in the program. If the state was previously hit, a counter is incremented to indicate that the test has performed a redundant hit, i.e., it is testing behaviour that has already been tested. Therefore, after all the tests in the suite have been executed and coverage was calculated, the statistics of each test will indicate whether the test is a redundant test or not. It is the user's responsibility to decide whether these tests should remain in the suite, or whether they may be removed.

It is important to note that the sequence in which the tests are executed have a great impact 
on the statistics of the tests. Take, for example, two tests, testA and testB, that test a similar behaviour of a program. If testA is executed first, then B may be marked as a redundant test, and vice versa. There may also be situations where redundant tests are desirable, as the tests may test a specific behaviour in conjunction with other behaviours that may have already been tested in other scenarios. ATCO only provides the statistics that indicate whether the tests hit any states that were not previously reached by other tests.

\subsubsection{Optimisation}

The information analysis phase consists of numerous time-consuming tasks. Some optimisation steps have, therefore, been taken to reduce the execution time of this phase.

\section{Concurrency Considerations}

Because tests are executed concurrently, and because more than one test may test the same behaviour of a program, it is possible that more than one test may be executing the same class in parallel. It is, therefore, possible that a race condition may occur with the coverage counters of some states in a SET. A race condition occurs when a process attempts to access or modify data while it is being modified by another process. This may cause inconsistencies in the data, which would result in erroneous data. To protect the coverage data of the SET, Java's synchronisation is used to ensure that only one process may read or modify the coverage counter of a specific state. Therefore, multiple processes may update the coverage counters of different states in a single $S E T$, but only one process may update the counter of a single state.

Java's method synchronisation idiom was chosen to protect against race conditions, because the resource requirements to assign a copy of all the SETs of large and complex programs may be too large for the available resources. Synchronisation may, however, have a negative impact on the execution time of concurrent processes, as a process may be blocked from completing its assigned task, if another process is currently accessing the state it requires.

\section{Constraint Solving Reduction}

The purpose of constraint solving during coverage calculation was discussed in an earlier section: constraint solving is used to determine which symbolic state was hit during test execution. Constraint solving reduction was applied to this process, by only performing constraint solving 
when a single statement has multiple symbolic states representing it. Therefore, when a statement has only one symbolic state representing it, that symbolic state will be the state that was hit during test execution, and thus constraint solving is unnecessary.

The number of constraint solving operations may be reduced even further, at the cost of test redundancy detection. For test redundancy detection, the actual values of the coverage counters are relevant. This is because test redundancy detection needs to be aware of how many times each statement was hit. When test redundancy detection is not required, the only relevant information is whether a statement was hit at least once. Therefore, when a symbolic state has been marked as hit for the first time, i.e., its coverage counter was incremented, all subsequent constraint solving operations on that symbolic state becomes irrelevant, since it is already covered. Therefore, when test redundancy detection is not measured, constraint solving will only be performed on a symbolic state if the statement it represents has multiple symbolic states representing it, and the symbolic state in question has not already been hit during testing. Whether the redundancy of tests should be measured during coverage calculation is configurable in $A T C O$.

\subsubsection{Execution Tracing of Manual Testing}

It was mentioned earlier that execution tracing is used during the execution of the JUnit tests in order to calculate coverage. One of the benefits of execution tracing is that any Java application, executed within a $J D I$ controlled $J V M$, may be traced. Therefore, coverage calculation does not have to be limited to the execution of JUnit test cases only. Allowing a user to manually test an application, while performing execution tracing on that manual test, allows coverage to be calculated on a manual test plan, i.e., a document, containing steps to follow, to test an application. Performing coverage calculation on manual testing allows application testers to verify the completeness of a test plan.

\subsection{Result Analysis Phase}

After coverage calculation is completed, as discussed in Section 3.3, the resulting SETs contain the coverage statistics of all statements, branches, and so on. These statistics are then used in the last phase of $A T C O$ 's execution, namely the result analysis phase. This phase involves 
guiding Artemis's test generation engine to generate tests for all the branches that are not covered by the existing test suite.

\subsubsection{Artemis' Test Generation Engine}

As mentioned earlier, Artemis detects unhandled run-time exceptions, and generates JUnit tests to verify the soundness of these detected exceptions. To accomplish the test generation, Artemis has a test generation engine that is used to convert a symbolic state, together with its path condition, into a JUnit test. This involves solving the constraints on the path condition of the symbolic state, and using the results of the solved path condition as input parameters for the method that contains the unhandled run-time exception.

\subsubsection{Test Generation in $A T C O$}

The test generation engine is used in $A T C O$ to generate tests for symbolic states that were not hit during coverage calculation, instead of generating tests for symbolic states that represent statements that cause the unhandled exceptions.

\subsection{Eclipse Plug-in}

Analysis tools are, more often than not, difficult to set up and run. There are long descriptions on dependencies, setup requirements, like environment variables, long classpaths, and, often, complex build scripts are needed, to run these tools. With Integrated Development Environments (IDEs), integrating functionality into an easy-to-use interface to speed up compilation, testing, and deploying of programs, why not attempt to integrate such an analysis tool into the $I D E$ as well? This would greatly reduce the effort to use such a tool, thereby encouraging developers to use such tools more frequently.

Eclipse is an open-source IDE with an extensible plug-in system. It consists of a run-time core that launches the platform base and then dynamically runs other plug-ins. The entire architecture of Eclipse, including the core, is comprised of plug-ins, allowing developers to completely remodel and customise Eclipse, should they so desire. This makes Eclipse ideal for integrating an analysis tool, such as ATCO. 
ATCO extends three of the standard plug-ins within Eclipse, namely the Resource Management plug-in, the Workbench plug-in, and the run-time core plug-in.

\subsubsection{Resource Management Plug-in}

As the name suggests, the resource management plug-in manages all the resources the user is working with. Resources include projects, folders, and files present in the Eclipse workspace. The workspace is where all the user's data files reside. Information on all resources within the workspace, all elements such as libraries, dependencies with other projects, etc. can be accessed through the resource management plug-in. The user can define a workspace directory on the file system to house projects, but the resources within the workspace are not limited to projects within that directory. Eclipse allows projects and other resources from arbitrary locations on the file system, or perhaps even on another machine, to be imported into the Eclipse workspace without physically copying it into the workspace directory.

The functionality provided by this plug-in is useful for a tool such as ATCO. It allows the tool to dynamically build classpaths for files to be analysed, by accessing the project's library list, and adding the locations of all libraries to the classpath, before running Soot during the information gathering phase. The resource management plug-in allows ATCO to access information on resources across the entire workspace, even if those resources are not physically stored in a single location. Therefore, apart from being able to handle projects within the workspace directory, $A T C O$ can also handle projects within the workspace that reside outside the workspace directory.

\subsubsection{Workbench Plug-in}

The workbench is the User Interface $(U I)$ plug-in that contains most of the $U I$ components in Eclipse. It defines the extension points that other plug-ins can use to contribute to the $U I$ of Eclipse. Some of the extension points include preferences, views, and the tool bar.

\section{Preferences}

When using a plug-in within an IDE, the user would expect a level of customisation. Plug-ins, therefore, allow a user to control the behaviour of the plug-in, to a certain extent, by presenting the user with a settings, or preferences, menu. The workbench provides some support for this 


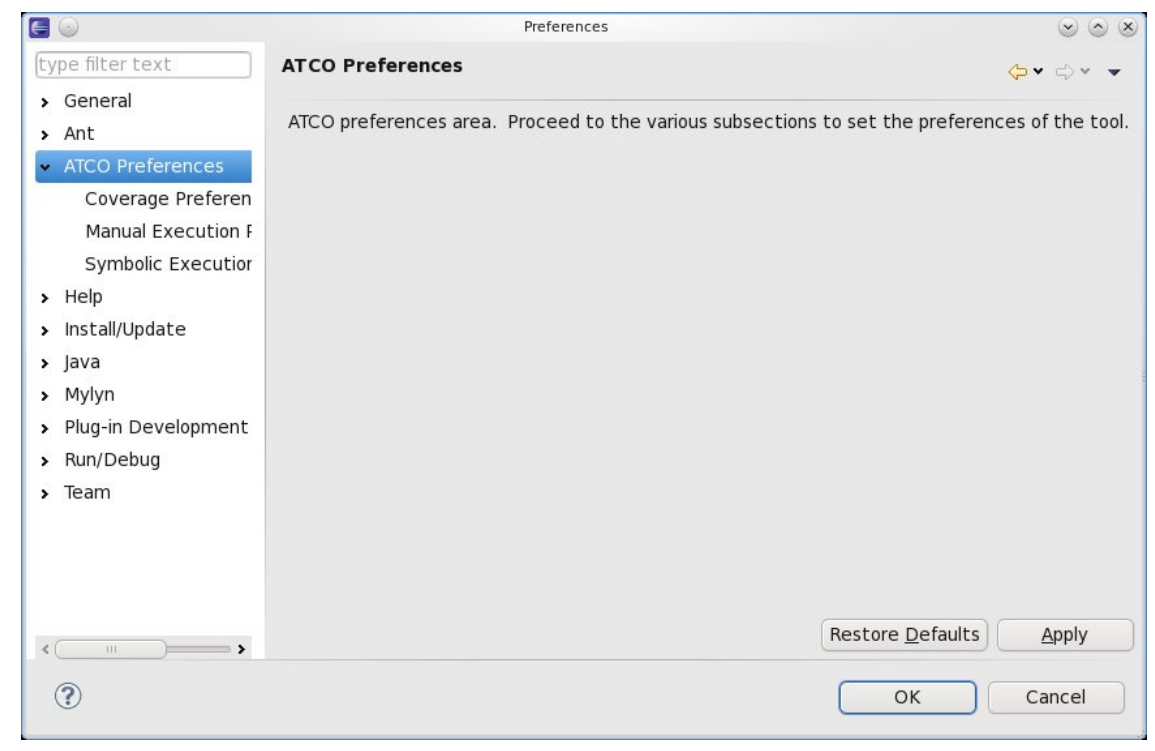

Figure 3.13: The main preferences page that houses the other preferences pages as sub pages.

through the Preferences dialog. The preferences dialog allow the user to customise the plug-in, and let these settings persist, even if Eclipse is terminated and launched again. This greatly improves the usability of a tool.

ATCO contains three preference pages: the Symbolic Execution Preferences (Figure 3.14, p. 69), the Coverage Preferences (Figure 3.15, p. 70), and the Manual Execution Preferences (Figure 3.16, p. 70), all grouped under a parent preference page (Figure 3.13, p. 68). These preference pages contain all the settings the user may use to configure ATCO. Settings for symbolic execution include the classes to be analysed, the interprocedural call depth, and the loop count limiter when symbolically executing the classes. The user may also specify the output directory where all debugging and graph files are stored. Settings for coverage calculation include selecting the tests to run, how many concurrent threads to use and how much memory to allocate to each thread, and the flag to indicate whether redundancy detection should be used during coverage calculation. Settings for manual execution include specifying the class to execute, together with the $J V M$ parameters and the command-line parameters to send to the class. The input required from the user is minimal, since all other information is extracted from the resource management plug-in.

A filtering implementation was added to the file selection component of the preferences pages, to filter the files to display. Within the symbolic execution preferences, only Java class files are displayed, since these are the only files ATCO can analyse. In the coverage preferences, 


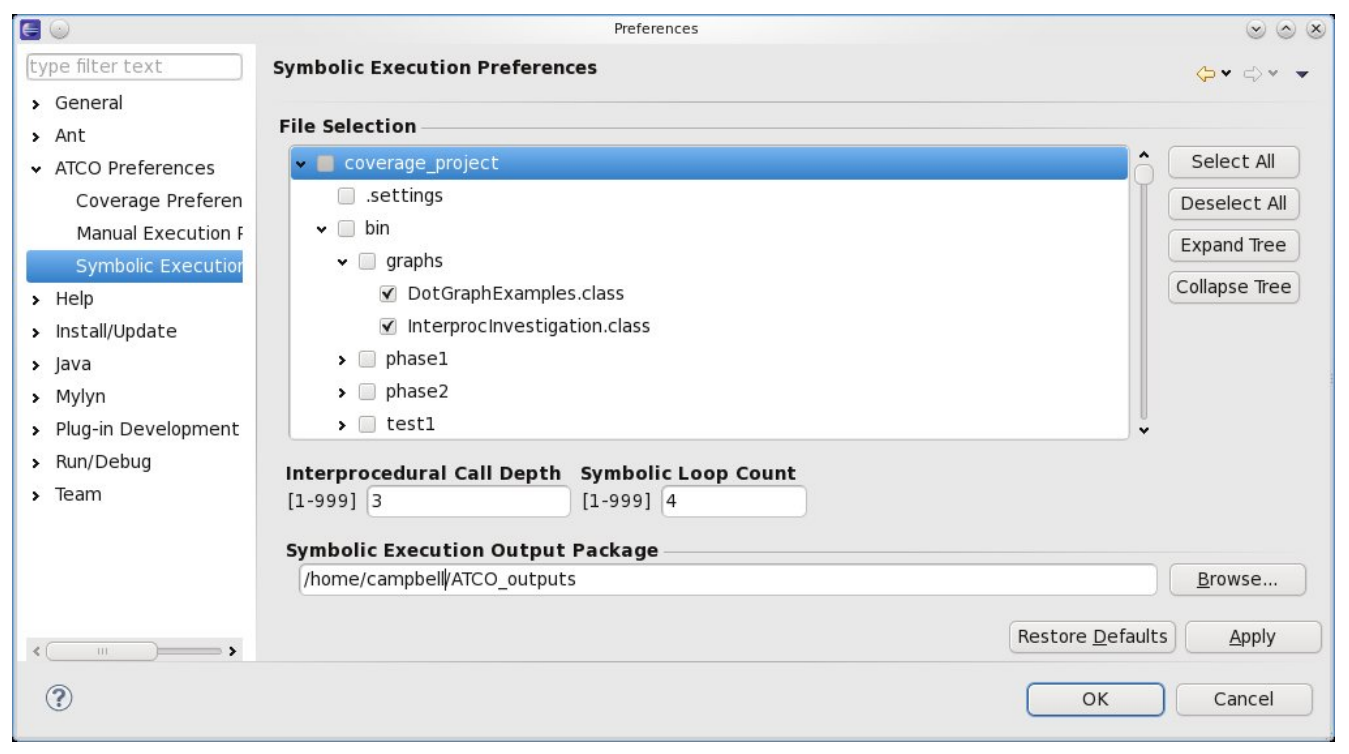

Figure 3.14: The symbolic execution preferences page.

only files that import the JUnit library are displayed, thereby limiting the user's selection of files to files that are likely to be actual JUnit tests. To improve usability when selecting multiple files with the file selection interface, recursive selection was implemented. When a folder is ticked or unticked, then all objects within that folder are ticked or unticked as well. Thus, ticking the project will select all applicable files within the entire file structure of the project.

The preferences pages of $A T C O$ are listed under the general Eclipse preferences (see Figure 3.17 , p. 70) since $A T C O$ is not project specific, but spans across the entire workspace. Keeping the settings uniform across the whole workspace, this allows the user to test multiple projects simultaneously, without having to do the setup for each project individually. However, this prevents individual $A T C O$ configurations for the various projects within the workspace.

\section{Views}

A View is a component that provides a visual output of information. A common example within Eclipse is the Problems view that lists compilation errors and warnings of the projects in the workspace. This is the component $A T C O$ uses to display the classes to be analysed, as well as the result of the analysis, after it is finished.

ATCO contains two views. First, the File Coverage View, shown in Figure 3.18, p. 71, 


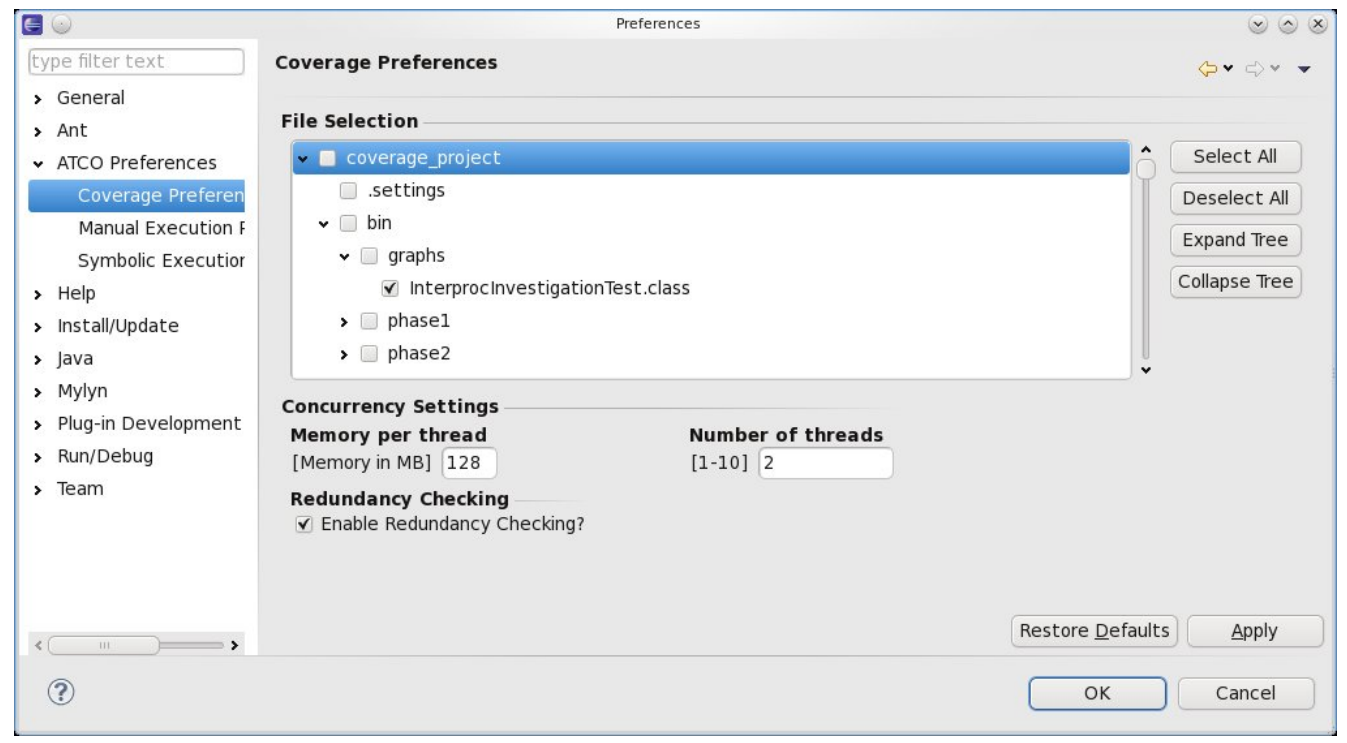

Figure 3.15: The coverage preferences page.

\begin{tabular}{|c|c|c|c|}
\hline e & \multirow[b]{2}{*}{ Manual Execution Preferences } & & \multirow{2}{*}{$\begin{array}{l}\Leftrightarrow \curvearrowright \otimes \\
\diamond \vee \Leftrightarrow \vee \vee\end{array}$} \\
\hline type filter text & & & \\
\hline > General & \multirow{2}{*}{\multicolumn{3}{|c|}{$\begin{array}{l}\text { Main Class } \\
\text { package.Class phase2.manual.UserDrivenAppMain }\end{array}$}} \\
\hline$>$ Ant & & & \\
\hline Coverage Preferen & \multicolumn{3}{|l|}{ JVM Arguments } \\
\hline Manual Execution : & \multicolumn{3}{|l|}{-ea -esa } \\
\hline Symbolic Executior & \multirow{2}{*}{\multicolumn{3}{|c|}{$\begin{array}{l}\text { Program Arguments } \\
\text { param1 param2 }\end{array}$}} \\
\hline > Help & & & \\
\hline > Install/Update & & & \\
\hline > Java & & & \\
\hline > Mylyn & & & \\
\hline > Plug-in Development & & & \\
\hline > Run/Debug & & & \\
\hline > Team & & & \\
\hline$\langle\longrightarrow$ & & Restore Defaults & Apply \\
\hline (?) & & OK & Cancel \\
\hline
\end{tabular}

Figure 3.16: The manual execution preferences page.

New Window
New Éditor
Open Perspective
Show View
Customize Perspective...
Save Perspective As...
Reset Perspective...
Close Perspective
Close All Perspectives
Navigation
Preferences

Figure 3.17: The standard Window drop down menu in Eclipse houses the preferences page. 


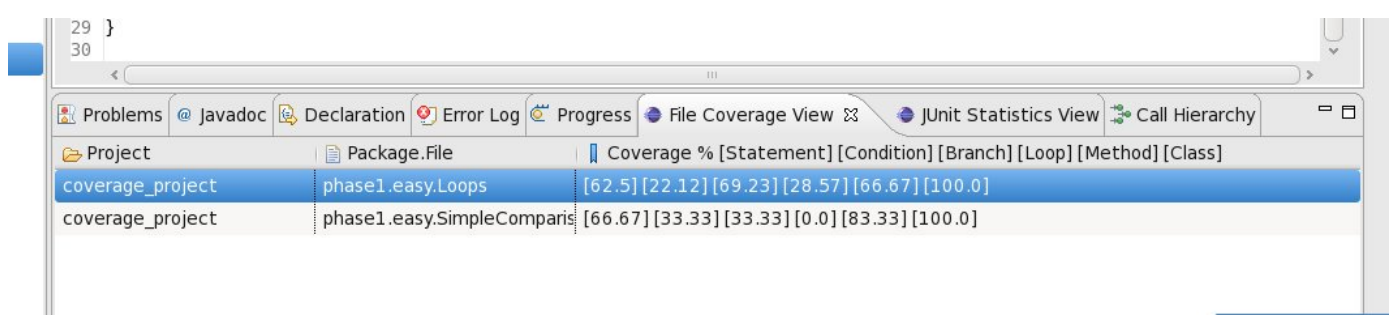

Figure 3.18: The file coverage view, displaying all the classes under analysis, together with their coverage results after analysis.

displays the coverage statistics of all the classes under test, and contains three columns. The Project column indicates the project of the selected file. The Package.File column shows the package and name of the selected class. The Coverage $\%$ column shows the coverage results as calculated by $A T C O$ after analysis. The user can sort the view results according to project name, or package.class name. For a more detailed summary of the coverage results, the user can double-click on the desired class, resulting in a pop-up window displaying a more detailed report on the coverage results. This pop-up is displayed in Figure 3.19, p. 72. The results have the class-wide statistics displayed at the top, followed by the statistics per each method within the class. Second, the JUnit Statistics View, shown in Figure 3.20, p. 72, displays the redundancy statistics of the tests used during analysis. The structure of the view is similar to the File Coverage View, displaying project, package, and class name of the selected tests. The Branches tested \% column indicates the percentage of branches across all the selected classes under test. This gives the user an indication on what percentage of the program under analysis a given JUnit test case tests. For a more detailed summary of the results, the user can double-click on the desired test, resulting in a pop-up window displaying the statistics per-class-per-method the selected test case tested. This pop-up is displayed in Figure 3.21, p. 72. Within the JUnit Statistics View, the columns indicate the number of branches that were newly hit (Hit), redundantly hit (Redundant), and the total number of branches hit by this test (Total).

The views implemented for $A T C O$ are very basic, but Eclipse provides functionality for more complex outputs, including graphs for the statistics, as well as extending the editor to display coverage statistics at, e.g., each line, method, or class. Unfortunately, due to time constraints in the development of $A T C O$, this functionality was never incorporated into the plug-in. 


\begin{tabular}{|c|c|c|c|c|c|c|}
\hline (1) & & tistics for phase & e1.easy.Loo & & 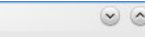 & $x$ \\
\hline Class-wide & & & & & & \\
\hline Coverage & Covered & Uncovered & Skipped & Total & Percentage & \\
\hline Statement & 20 & 12 & 0 & 32 & 62.5 & \\
\hline Condition & 23 & 81 & 0 & 104 & 22.12 & \\
\hline Branch & 9 & 4 & 0 & 13 & 69.23 & \\
\hline Loop & 2 & 5 & 0 & 7 & 28.57 & \\
\hline Method & 4 & 2 & 0 & 6 & 66.67 & \\
\hline Class & 1 & 0 & 0 & 1 & 100.0 & \\
\hline void $<$ init $>$ ( & & & & & & \\
\hline Coverage & Covered & Uncovered & Skipped & Total & Percentage & \\
\hline Statement & 1 & 0 & 0 & 1 & 100.0 & \\
\hline Condition & 0 & 0 & 0 & 0 & 0.0 & \\
\hline Branch & 0 & 0 & 0 & 0 & 0.0 & \\
\hline Loop & 0 & 0 & 0 & 0 & 0.0 & \\
\hline Method & 1 & 0 & 0 & 1 & 100.0 & \\
\hline Class & 1 & 0 & 0 & 1 & 100.0 & \\
\hline void methoc & d4(int, int & & & & & \\
\hline Coverage & Covered & Uncovered & Skipped & Total & Percentage & \\
\hline Statement & 7 & 0 & 0 & 7 & 100.0 & \\
\hline Condition & 13 & 31 & 0 & 44 & 29.55 & v \\
\hline
\end{tabular}

Figure 3.19: The detailed view available from the file coverage view by double-clicking on any of the classes in the view.

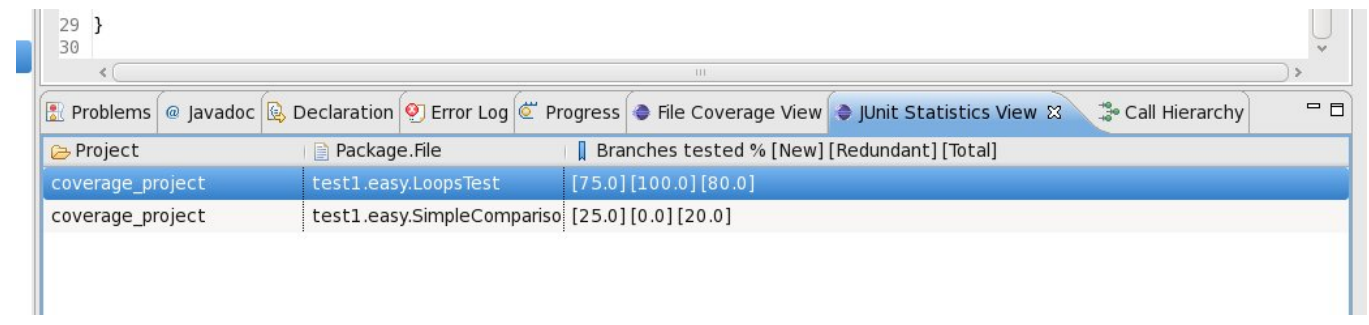

Figure 3.20: The JUnit statistics view, displaying all the selected JUnit test cases, together with their redundancy results after analysis.

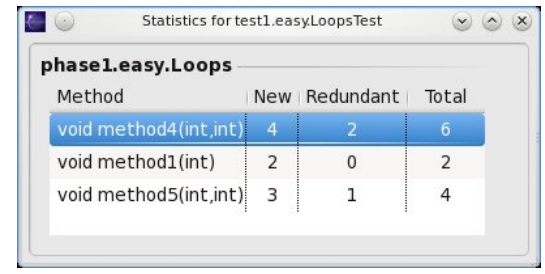

Figure 3.21: The detailed view available from the JUnit statistics view by double-clicking on any of the test cases in the view. 


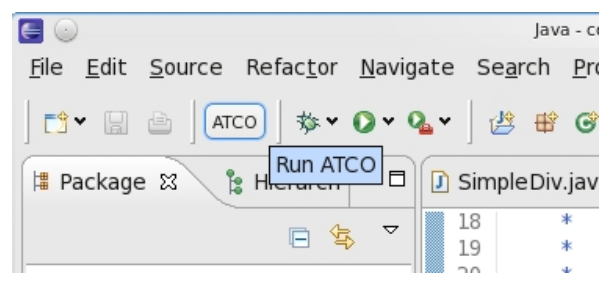

Figure 3.22: The $A T C O$ action button, housed within its own tool bar group, and complete with tool-tip, used to launch the analysis of the selected class(es). For lack of an icon for the button, the tool name was used.

\section{Tool Bar Action Button}

The workbench contains many extension points for adding action buttons, which perform an action when pressed. These buttons can be added to many places in the workbench, from the views, to drop-down menus, the tool bar, and so on. These action buttons, when clicked, execute the code embedded within the class used to implement the button.

Within $A T C O$, the implemented action button is used to launch its entire analysis process. The user may set the preferences of $A T C O$ through its preferences pages, then click the action button to run the analysis on the selected classes. Within the action button, the arguments, to be passed to Soot, are constructed by using the resource management plug-in to locate the required libraries and source locations, and compiling all the necessary information into a valid Soot argument string to be passed to Artemis. Artemis is then invoked with this argument string, which is then followed by the analysis implemented in ATCO. Figure 3.22, p. 73 shows the action button with tool-tip.

\subsubsection{Jobs}

One of the challenges of plugging a processor intensive tool into an IDE is that the tool must be able to run concurrently with the other operations constantly running within the IDE. Merely running the tool will seize the other functions of the $I D E$, such as the various views, compilers, and even the editors. This issue holds throughout the entire Eclipse environment, since it constantly has plug-ins running various tasks at all times. Eclipse addresses this issue with its concurrency infrastructure by introducing the Jobs package. A job represents a unit of work that needs to be able to run asynchronously. A plug-in creates and schedules a job, which is then added to the job queue maintained by the Eclipse platform. 


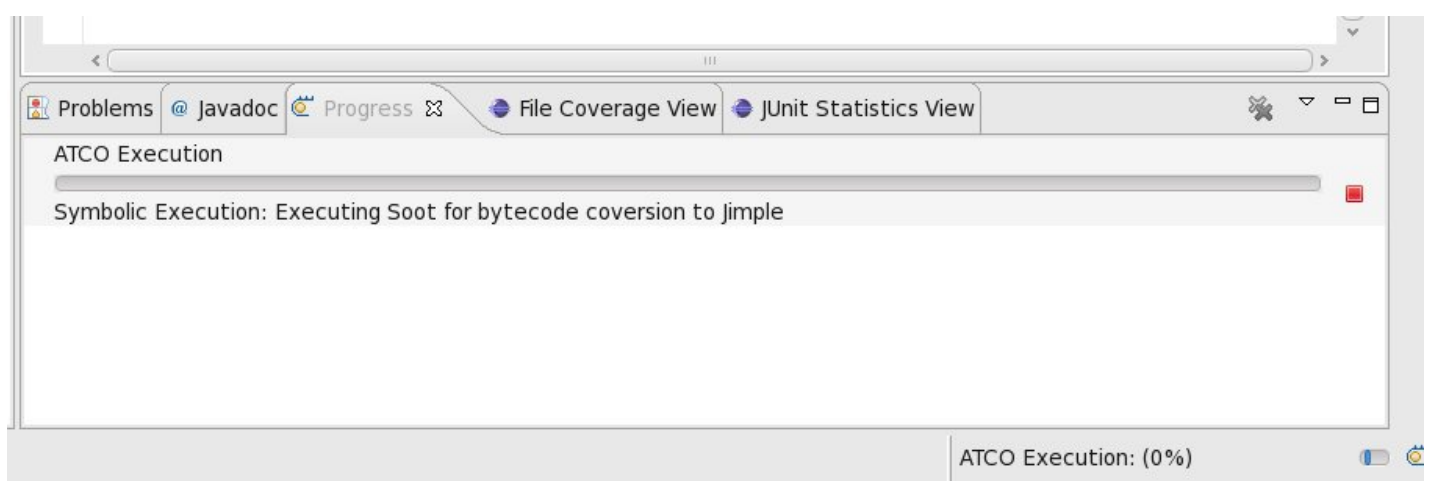

Figure 3.23: The progress bar indicating the overall progress of the various execution phases within $A T C O$. This example displays the information gathering phase, where Soot converts the Java bytecode into Jimple format, and where symbolic execution will be performed.

The analysis in $A T C O$ is run within a job object. This allows the processor intensive operation of symbolic execution and constraint solving to execute concurrently with the rest of Eclipse, allowing the user to still use the IDE during analysis.

Another feature provided by the job package is the Progress Monitor, shown in Figure 3.23 , p. 74 . This may be used to output statistics on the progress of the job being executed under the Progress view, which is standard to Eclipse. ATCO use the progress monitor to display the phase it is currently executing, the class currently being analysed, and so on. The concurrent execution of the information analysis phase makes the use of the progress monitor difficult, since there is only one progress monitor for each job. Therefore, the progress monitor is used to display the progress across all classes analysed, i.e., the percentage of classes already analysed, as opposed to displaying the progress of each class currently being analysed. 


\section{Chapter 4}

\section{Evaluation}

Previous chapters presented the theory, as well as the design and implementation of the ATCO plug-in. This chapter will describe the various experiments that were used to evaluate ATCO. The experiments were designed with the following objectives in mind.

Correctness of Coverage Calculation: The correctness of coverage calculation with ATCO, i.e., whether the coverage calculation, measured with $A T C O$, is correct, needs to be evaluated. The impact that the analysis configurations in ATCO have on the accuracy of the coverage calculation, needs to be evaluated as well. The analysis may be configured by changing any of the following values (default values are included in parentheses): call depth (1), loop count (4), and whether redundancy detection is turned on (yes).

Performance of Coverage Calculation: The performance of coverage calculation with ATCO needs to be evaluated. The impact of the concurrent configuration in $A T C O$, i.e., the number of concurrent threads used during coverage calculation, needs to be evaluated as well.

Effectiveness of Generated Test Cases: The effectiveness and correctness of the test cases, generated through ATCO's analysis, need to be evaluated. The impact of the analysis configurations on the effectiveness of the generated test cases needs to be evaluated as well. 


\subsection{Test Setup}

$A T C O$ has been developed as an Eclipse plug-in to take advantage of Eclipse's Graphical User Interface $(G U I)$ extensions, as was described in the previous chapter. However, the tool was also developed to allow it to be executed from a normal command-line interface. The purpose of this is to allow ATCO to be executed on two different environments, both of which will be discussed here.

\subsubsection{Single-core GUI Environment}

Eclipse is a GUI application, and, therefore, require an environment that is able to display its interfaces. Most standard operating systems, such as Microsoft Windows and Linux, are capable of displaying GUI applications. However, the GUI environment available for the evaluation of the ATCO plug-in is a single-core machine with limited resources. This environment is, therefore, only used during testing of the ATCO plug-in's GUI. All experiments, which are presented in this chapter, are performed on a multi-core command-line environment.

\subsubsection{Multi-core Command-Line Environment}

To fully utilise the concurrent design in $A T C O$, a multi-core system is necessary. The multi-core system, used for all of the experiments, contains two $2.26 \mathrm{GHz}$ Intel Xeon E5520 processors, which supports up to a total of 8 threads, with $4 \mathrm{~GB}$ of allocated memory. However, this environment is only accessible remotely via secure shell $(S S H)$. Therefore, Eclipse could not be used in this environment. Thus, ATCO is adapted to allow it to execute on a command-line interface, in order to use this environment.

\subsection{Other Tools}

There are a number of tools that generate test cases for the purpose of maximising coverage, like JTest [27] and Symstra [44]. However, these tools do not consider an already existing JUnit test suite. These tools generate an additional, independent test suite. As the focus of this thesis is coverage calculation, and using that information to generate tests, it is decided that little value would be received from comparing $A T C O$ to these tools. Instead, the experiments, described in this chapter, will be compared to EMMA [17], a popular coverage calculation tool 
discussed in Section 2.8.4, p. 39. EMMA is the chosen tool because it is an open-source, freely available coverage calculation tool, that is well documented and easy to use. It also presents its results in a manner that makes it easy to compare to ATCO.

\subsection{Correctness of Coverage Calculation}

The first set of experiments aims to verify whether ATCO measures coverage correctly. This is separated into three groups of tests. First, a set of custom applications, together with JUnit test cases. Second, a set of small, real-world applications, often used to demonstrate the usage of the JUnit test framework. Third, a large, real-world application, often used in evaluations of program analysis and program verification tools.

\subsubsection{Custom Applications}

The purpose of the custom applications is to allow for accurate, manual confirmation that ATCO yields the expected results. These custom applications are also used to evaluate the impact of the analysis configurations. These results are compared to the results generated by EMMA [17], to further verify the correctness of ATCO.

There are three custom applications used for this evaluation:

- SimpleComparisons: This application is a single class that consists of independent methods. Thus, no method within the class invokes another method, so its coverage results are unaffected by the configured call depth. It also contains no loops, so its coverage results are unaffected by the configured loop count.

- InterprocInvestigations: This application is a single class that consists of methods that invoke other methods in the class. The methods also have varying scopes, i.e., public, private, and protected methods. This application is used to evaluate the effect of various call depth configurations on the $S E T$ and the coverage calculation results. It also demonstrates the benefits of interprocedural analysis on methods that cannot be directly invoked outside of their scope. It contains no loops, so its coverage results are unaffected by the configured loop count.

- Loops: This application is a single class that consists of independent methods that contain loops. This application is used to evaluate the effect of various loop count configurations 
on the $S E T$ and the coverage calculation results. Since the methods are independent, the results are unaffected by the configured call depth.

Each of the above-mentioned custom applications have corresponding JUnit test cases, used to test the application. These test cases are designed to follow specific paths through the various methods in the applications. Knowledge of the specific paths, gained through manual inspection of the applications and their JUnit tests, allow the results to be verified manually.

\section{Results}

The results in Table 4.1, p. 79, displays the accuracy of $A T C O$ and the impact of the different analysis configurations. The results are verified as correct and accurate through manual inspection of the source code of the custom applications, together with the JUnit test cases. Further confidence in the results, presented by $A T C O$, is gained by using EMMA to measure coverage on the custom applications as well. The comparable results between ATCO and EMMA are displayed in Table 4.2, p. 80. EMMA rounds its results to the nearest integer value. Therefore, for the sake of comparison, ATCO's coverage results are also rounded to the nearest integer value.

\section{Evaluation}

Manual code inspection of the experiments in Table 4.1, p. 79, together with the comparison to EMMA in Table 4.2, p. 80, provides sufficient evidence that ATCO yields the correct results during coverage calculation. The results in Table 4.1 will now be discussed in more detail.

- SimpleComparisons: This custom application contains no interprocedural method calls, no symbolic loops, and no nested or sequential symbolic branching statements. Therefore, each statement has, at most, one symbolic state representing it. As such, no constraint solving is required during coverage calculation, since constraint solving is only required when a single statement is represented by multiple symbolic states. As no constraint solving occurs during coverage calculation, there is also no benefit from disabling redundancy detection.

- InterprocInvestigations: An increase in explored symbolic states is observed, when comparing the intraprocedural analysis, i.e., $C D=0$, to interprocedural analysis, i.e., $C D>$ 


\begin{tabular}{|c|c|c|c|c|c|c|c|c|c|c|}
\hline \multicolumn{11}{|c|}{ SimpleComparisons } \\
\hline CD & $\mathrm{LC}$ & RD & St & CS & $\mathrm{Cl}$ & Me & $\mathrm{Br}$ & Ln & Lo & TR \\
\hline $\mathrm{N} / \mathrm{A}$ & $\mathrm{N} / \mathrm{A}$ & Yes & 60 & 0 & $100 \%$ & $83.33 \%$ & $62.5 \%$ & $76.19 \%$ & $\mathrm{~N} / \mathrm{A}$ & $54.55 \%$ \\
\hline $\mathrm{N} / \mathrm{A}$ & $\mathrm{N} / \mathrm{A}$ & $\mathrm{No}$ & 60 & 0 & $100 \%$ & $83.33 \%$ & $62.5 \%$ & $76.19 \%$ & $\mathrm{~N} / \mathrm{A}$ & $\mathrm{N} / \mathrm{A}$ \\
\hline \multicolumn{11}{|c|}{ InterprocInvestigations } \\
\hline CD & $\mathrm{LC}$ & RD & St & $\mathrm{CS}$ & $\mathrm{Cl}$ & $\mathrm{Me}$ & $\mathrm{Br}$ & Ln & Lo & TR \\
\hline 0 & $\mathrm{~N} / \mathrm{A}$ & Yes & 161 & 0 & $100 \%$ & $100 \%$ & $61.54 \%$ & $78.26 \%$ & $\mathrm{~N} / \mathrm{A}$ & $41.46 \%$ \\
\hline 0 & $\mathrm{~N} / \mathrm{A}$ & No & 161 & 0 & $100 \%$ & $100 \%$ & $61.54 \%$ & $78.26 \%$ & $\mathrm{~N} / \mathrm{A}$ & $\mathrm{N} / \mathrm{A}$ \\
\hline 1 & $\mathrm{~N} / \mathrm{A}$ & Yes & 225 & 39 & $100 \%$ & $100 \%$ & $61.54 \%$ & $78.26 \%$ & $\mathrm{~N} / \mathrm{A}$ & $43.90 \%$ \\
\hline 1 & $\mathrm{~N} / \mathrm{A}$ & No & 225 & 27 & $100 \%$ & $100 \%$ & $61.54 \%$ & $78.26 \%$ & $\mathrm{~N} / \mathrm{A}$ & $\mathrm{N} / \mathrm{A}$ \\
\hline 2 & $\mathrm{~N} / \mathrm{A}$ & Yes & 227 & 43 & $100 \%$ & $100 \%$ & $61.54 \%$ & $78.26 \%$ & $\mathrm{~N} / \mathrm{A}$ & $43.90 \%$ \\
\hline 2 & $\mathrm{~N} / \mathrm{A}$ & $\mathrm{No}$ & 227 & 31 & $100 \%$ & $100 \%$ & $61.54 \%$ & $78.26 \%$ & $\mathrm{~N} / \mathrm{A}$ & $\mathrm{N} / \mathrm{A}$ \\
\hline 3 & $\mathrm{~N} / \mathrm{A}$ & Yes & 227 & 43 & $100 \%$ & $100 \%$ & $61.54 \%$ & $78.26 \%$ & $\mathrm{~N} / \mathrm{A}$ & $43.90 \%$ \\
\hline 3 & $\mathrm{~N} / \mathrm{A}$ & $\mathrm{No}$ & 227 & 31 & $100 \%$ & $100 \%$ & $61.54 \%$ & $78.26 \%$ & $\mathrm{~N} / \mathrm{A}$ & $\mathrm{N} / \mathrm{A}$ \\
\hline \multicolumn{11}{|c|}{ Loops } \\
\hline CD & $\mathrm{LC}$ & RD & St & $\mathrm{CS}$ & $\mathrm{Cl}$ & $\mathrm{Me}$ & $\mathrm{Br}$ & Ln & Lo & TR \\
\hline $\mathrm{N} / \mathrm{A}$ & 2 & Yes & 198 & 116 & $100 \%$ & $100 \%$ & $69.23 \%$ & $100 \%$ & $85.71 \%$ & $80.6 \%$ \\
\hline $\mathrm{N} / \mathrm{A}$ & 2 & $\mathrm{No}$ & 198 & 62 & $100 \%$ & $100 \%$ & $69.23 \%$ & $100 \%$ & $0 \%$ & $\mathrm{~N} / \mathrm{A}$ \\
\hline $\mathrm{N} / \mathrm{A}$ & 4 & Yes & 466 & 329 & $100 \%$ & $100 \%$ & $76.92 \%$ & $100 \%$ & $85.71 \%$ & $81.51 \%$ \\
\hline $\mathrm{N} / \mathrm{A}$ & 4 & No & 466 & 232 & $100 \%$ & $100 \%$ & $76.92 \%$ & $100 \%$ & $0 \%$ & $\mathrm{~N} / \mathrm{A}$ \\
\hline $\mathrm{N} / \mathrm{A}$ & 6 & Yes & 863 & 645 & $100 \%$ & $100 \%$ & $84.62 \%$ & $100 \%$ & $85.71 \%$ & $82.74 \%$ \\
\hline $\mathrm{N} / \mathrm{A}$ & 6 & $\mathrm{No}$ & 863 & 506 & $100 \%$ & $100 \%$ & $84.62 \%$ & $100 \%$ & $0 \%$ & $\mathrm{~N} / \mathrm{A}$ \\
\hline $\mathrm{N} / \mathrm{A}$ & 8 & Yes & 1392 & 1065 & $100 \%$ & $100 \%$ & $84.62 \%$ & $100 \%$ & $85.71 \%$ & $83.67 \%$ \\
\hline $\mathrm{N} / \mathrm{A}$ & 8 & $\mathrm{No}$ & 1392 & 901 & $100 \%$ & $100 \%$ & $84.62 \%$ & $100 \%$ & $0 \%$ & $\mathrm{~N} / \mathrm{A}$ \\
\hline $\mathrm{N} / \mathrm{A}$ & 10 & Yes & 2050 & 1589 & $100 \%$ & $100 \%$ & $92.31 \%$ & $100 \%$ & $85.71 \%$ & $84.07 \%$ \\
\hline $\mathrm{N} / \mathrm{A}$ & 10 & $\mathrm{No}$ & 2050 & 1399 & $100 \%$ & $100 \%$ & $92.31 \%$ & $100 \%$ & $0 \%$ & $\mathrm{~N} / \mathrm{A}$ \\
\hline
\end{tabular}

Table 4.1: The correctness evaluation of ATCO through custom applications. Each table header contains the name of the custom application analysed. The columns contain the configured call depth $(\mathbf{C D})$, the configured loop count $(\mathbf{L C})$, whether redundancy detection was used (RD), the number of explored symbolic states (St), the number of constraint solving operations performed during the information gathering phase and information analysis phase (CS), and the following coverage statistics: Class coverage $(\mathbf{C l})$, Method coverage $(\mathbf{M e})$, Branch coverage $(\mathbf{B r})$, Line coverage $(\mathbf{L n})$, and Loop coverage $(\mathbf{L o})$. The last column contains the test redundancy statistics (TR), indicating the percentage of states, hit during testing, that were already hit, i.e., redundant. 


\begin{tabular}{|c|c|c|c|c|c|c|}
\hline \multicolumn{7}{|c|}{ SimpleComparisons } \\
\hline & \multicolumn{2}{|c|}{ Class } & \multicolumn{2}{|c|}{ Method } & \multicolumn{2}{|c|}{ Line } \\
\hline Tool & Total & Cov & Total & Cov & Total & Cov \\
\hline$A T C O$ & 1 & $100 \%$ & 6 & $83 \%$ & 21 & $76 \%$ \\
\hline$E M M A$ & 1 & $100 \%$ & 6 & $83 \%$ & 21 & $76 \%$ \\
\hline \multicolumn{7}{|c|}{ InterprocInvestigations } \\
\hline & \multicolumn{2}{|c|}{ Class } & \multicolumn{2}{|c|}{ Method } & \multicolumn{2}{|c|}{ Line } \\
\hline Tool & Total & Cov & Total & Cov & Total & Cov \\
\hline$A T C O$ & 1 & $100 \%$ & 18 & $100 \%$ & 69 & $78 \%$ \\
\hline$E M M A$ & 1 & $100 \%$ & 18 & $100 \%$ & 69 & $78 \%$ \\
\hline \multicolumn{7}{|c|}{ Loops } \\
\hline & \multicolumn{2}{|c|}{ Class } & \multicolumn{2}{|c|}{ Method } & \multicolumn{2}{|c|}{ Line } \\
\hline Tool & Total & Cov & Total & Cov & Total & Cov \\
\hline$A T C O$ & 1 & $100 \%$ & 6 & $100 \%$ & 30 & $100 \%$ \\
\hline$E M M A$ & 1 & $100 \%$ & 6 & $100 \%$ & 30 & $100 \%$ \\
\hline
\end{tabular}

Table 4.2: The correctness evaluation of ATCO, by comparing its results to that of EMMA. Each table header contains the name of the custom application analysed. The columns contain the tool used (Tool), and the total number (Total) of classes, methods, and executable lines found in the applications, as well as the corresponding percentage of this number to the total number of classes, methods, and executable lines that are covered (Cov).

0. Also, an increase in call depth increases the number of constraint solving operations required to accurately perform coverage calculation, since the invoked methods have multiple return states. A method that invokes another method gains an execution path for every possible return state in the method it invoked. Interprocedural analysis yields an increase in test redundancy, as the invoked methods are also considered, which increases the number of symbolic states that may be hit redundantly. Disabling redundancy detection yields a decrease in the number of constraint solving operations, but due to the small size of this application, the benefits are minimal. Larger examples will yield more significant benefits.

- Loops: A significant increase in explored symbolic states, together with a significant increase in constraint solving operations, is observed with an increase in loop count $(L C)$. This is to be expected, as the loop count dictates the number of times a symbolic loop is iterated during symbolic execution. A notable occurrence is that line coverage is at $100 \%$, while the branch coverage is less than $100 \%$. This occurs when the loop count is less than the number of times the loop is actually iterated during testing. The path where the test 
execution exits the loop is never represented symbolically. So, when the test execution exits the loop, no symbolic state contains those path conditions, and thus the loop exit branch is never hit during testing. The branch coverage increases with the increase in loop count, because the JUnit test cases iterate over all the loops variably between 1 and 10 times. Therefore, the increase in loop count reduces the instances where the loop count is less than the number of actual test iterations of a loop. Finally, the high test redundancy statistic is due to the interesting case with symbolic loops, as discussed in Section 3.3.3, p. 62. Test redundancy is measured on the symbolic states being hit during testing. And, as stated in Section 3.3.3, with every iteration of a loop during testing, all the branches representing that loop in the SET, that fall within the bounds of the actual loop, will be marked as a hit. This results in each test hitting many states repeatedly, which, in turn, causes the high test redundancy.

The custom applications, used to evaluate $A T C O$, all contain only primitive data types as method input parameters, and in the branching statements. The experiments indicate that this works well with symbolic execution and constraint solving. However, to evaluate the real-world use of $A T C O$, non-primitive data types need to be considered.

\subsubsection{Small Real-World Applications}

Three small, real-world examples of the JUnit test framework are used to evaluate ATCO. The purpose of these experiments are to evaluate $A T C O$ with small examples that are not custom applications, written specifically to test $A T C O$. These results are compared to the results generated by EMMA [17], to further verify the correctness of ATCO.

The three applications used for these experiments are:

- Latitude [24]: This application is a single class that houses latitude coordinate information. This is a simple application, with no interprocedural method calls or loops.

- ShoppingCart [6]: This is a simple example of a shopping cart application. It consists of two classes (ShoppingCart, and Product), and a custom exception (ProductNotFoundException). It contains some interprocedural method calls, and one loop, which will iterate at most twice, according to its test. 
- Money [29]: This is a simple example of a currency conversion system, with some basic calculations between currencies. It consists of two classes (Money, and MoneyBag), and one interface (IMoney). It contains many interprocedural method calls, and many loops.

\section{Results}

The results in Table 4.3, p. 82, display the accuracy and configuration impact of ATCO. EMMA is used to measure coverage on the small applications as well. The comparable results between ATCO and EMMA are displayed in Table 4.4, p. 83. As before, ATCO's coverage results are rounded to the nearest integer for the sake of comparison.

\begin{tabular}{|c|c|c|c|c|c|c|c|c|c|c|}
\hline \multicolumn{10}{|c|}{ Latitude } \\
\hline CD & LC & RD & St & CS & Cl & Me & Br & Ln & Lo & TR \\
\hline N/A & N/A & N/A & 57 & 0 & $100 \%$ & $100 \%$ & $80 \%$ & $95.24 \%$ & N/A & $25 \%$ \\
\hline \hline \multicolumn{10}{|c|}{ ShoppingCart } \\
\hline CD & LC & RD & St & CS & Cl & Me & Br & Ln & Lo & TR \\
\hline 0 & N/A & N/A & 161 & 0 & $100 \%$ & $90.91 \%$ & $50 \%$ & $75.86 \%$ & N/A & $25 \%$ \\
\hline 1 & N/A & N/A & 161 & 0 & $100 \%$ & $90.91 \%$ & $50 \%$ & $75.86 \%$ & N/A & $25 \%$ \\
\hline \hline \multicolumn{10}{|c|}{ Money } \\
\hline CD & LC & RD & St & CS & Cl & Me & Br & Ln & Lo & TR \\
\hline 0 & 4 & Yes & 602 & 2681 & $100 \%$ & $96.88 \%$ & $80 \%$ & $86.73 \%$ & $85.71 \%$ & $96.48 \%$ \\
\hline 0 & 4 & No & 602 & 103 & $100 \%$ & $96.88 \%$ & $80 \%$ & $86.73 \%$ & N/A & N/A \\
\hline 0 & 8 & Yes & 882 & 4653 & $100 \%$ & $96.88 \%$ & $80 \%$ & $86.73 \%$ & $85.71 \%$ & $96.27 \%$ \\
\hline 0 & 8 & No & 882 & 183 & $100 \%$ & $96.88 \%$ & $80 \%$ & $86.73 \%$ & N/A & N/A \\
\hline 1 & 4 & Yes & 6728 & 22478 & $100 \%$ & $96.88 \%$ & $80 \%$ & $86.73 \%$ & $85.71 \%$ & $90.39 \%$ \\
\hline 1 & 4 & No & 6728 & 2186 & $100 \%$ & $96.88 \%$ & $80 \%$ & $86.73 \%$ & N/A & N/A \\
\hline 1 & 8 & Yes & 1530380 & 4595462 & $100 \%$ & $96.88 \%$ & $80 \%$ & $86.73 \%$ & $85.71 \%$ & $88.89 \%$ \\
\hline 1 & 8 & No & 1530380 & 524546 & $100 \%$ & $96.88 \%$ & $80 \%$ & $86.73 \%$ & N/A & N/A \\
\hline
\end{tabular}

Table 4.3: The correctness evaluation of ATCO through small, real-world applications. Each table header contains the name of the application analysed. The columns contain the configured call depth $(\mathbf{C D})$, the configured loop count $(\mathbf{L C})$, whether redundancy detection was used (RD), the number of explored symbolic states (St), the number of constraint solving operations performed during the information gathering phase and information analysis phase (CS), and the following coverage statistics: Class coverage $(\mathbf{C l})$, Method coverage $(\mathbf{M e})$, Branch coverage $(\mathbf{B r})$, Line coverage $(\mathbf{L n})$, and Loop coverage $(\mathbf{L o})$. The last column contains the test redundancy statistics (TR), indicating the percentage of states, hit during testing, that were already hit, i.e., redundant. 


\begin{tabular}{|c|c|c|c|c|c|c|}
\hline \multicolumn{7}{|c|}{ Latitude } \\
\hline & \multicolumn{2}{|c|}{ Class } & \multicolumn{2}{c|}{ Method } & \multicolumn{2}{c|}{ Line } \\
\hline Tool & Total & Cov & Total & Cov & Total & Cov \\
\hline$A T C O$ & 1 & $100 \%$ & 3 & $100 \%$ & 21 & $95 \%$ \\
\hline$E M M A$ & 1 & $100 \%$ & 3 & $100 \%$ & 21 & $90 \%$ \\
\hline \hline
\end{tabular}

\begin{tabular}{|c|c|c|c|c|c|c|}
\hline \multicolumn{7}{|c|}{ ShoppingCart } \\
\hline & \multicolumn{2}{|c|}{ Class } & \multicolumn{2}{|c|}{ Method } & \multicolumn{2}{|c|}{ Line } \\
\hline Tool & Total & Cov & Total & Cov & Total & Cov \\
\hline$A T C O$ & 3 & $100 \%$ & 11 & $90 \%$ & 29 & $75 \%$ \\
\hline$E M M A$ & 3 & $100 \%$ & 11 & $100 \%$ & 29 & $97 \%$ \\
\hline \multicolumn{7}{|c|}{ Money } \\
\hline & \multicolumn{2}{|c|}{ Class } & \multicolumn{2}{|c|}{ Method } & \multicolumn{2}{|c|}{ Line } \\
\hline Tool & Total & Cov & Total & Cov & Total & Cov \\
\hline$A T C O$ & 2 & $100 \%$ & 32 & $96 \%$ & 98 & $86 \%$ \\
\hline$E M M A$ & 2 & $100 \%$ & 32 & $97 \%$ & 98 & $88 \%$ \\
\hline
\end{tabular}

Table 4.4: The correctness evaluation of $A T C O$, by comparing its results with that of EMMA. Each table header contains the name of the application analysed. The columns contain the tool used (Tool), and the total number (Total) of classes, methods, and executable lines found in the applications, as well as the corresponding percentage of this number to the total number of classes, methods, and executable lines that are covered (Cov).

\section{Evaluation}

First, the results, displayed in Table 4.3, p. 82, are discussed in detail.

- Latitude: Similar to the SimpleComparisons example in the custom application evaluation, this application is unaffected by the analysis configurations. Constraint solving never has to be used, as there are no statements that are represented by more than one symbolic state.

- ShoppingCart: This application contains some interprocedural method calls, but the results show no change, regardless of call depth. This is because the methods, invoked by other methods, do not contain any branches. Therefore, it does not contribute any additional paths to the invoking method, and thus the results remain unchanged across the configured call depths.

- Money: This application provides a clear example of, first, the state-explosion problem when using interprocedural analysis and symbolic loops, and second, the benefits of not 
performing redundancy detection. First, there is a significant increase in explored symbolic states across the configured call depths, and an even more significant increase across the configured loop counts. This is because the application contains methods, with symbolic loops, that invoke other methods with a number of execution paths. Since symbolic execution iterates a symbolic loop a number of times, and since interprocedural analysis follows all invoked methods, up to a certain depth, the state space grows exponentially. A notable observation is the reduction in test redundancy levels as the state space grows. Because test redundancy is measured on the symbolic states in the $S E T$, an increase in the state space increases the total number of states that may be hit during testing. Thus, if the state space grows, but the number of symbolic states that are hit redundantly remain the same, or at least increases at a slower rate, then the total test redundancy level will decrease. Second, disabling redundancy detection vastly reduces the number of constraint solving operations required to perform coverage calculation, showing up to a $96.16 \%$ reduction in constraint solving operations.

Second, the comparable results between $A T C O$ and EMMA, displayed in Table 4.4, p. 83 are presented and evaluated. These results all yield different results to EMMA. This is because the symbolic execution engine is still under development, and currently cannot handle non-primitive types properly. Also, constraint solving, by definition, is used to solve constraints of primitive variable types, such as binary or numeric data types. It is not for Objects, which are nonprimitive types. These small applications contain non-primitive input parameters to methods, and symbolic loops over Collections of non-primitive types. They also contain non-primitive conditions on branches, by using the instanceof operator in Java. As a result, constraint solving cannot solve some of the path conditions on symbolic states. Therefore, the information analysis phase cannot accurately determine which of the symbolic states, representing the statement in question, should be marked as hit. This results in no symbolic states being marked as covered, which causes the degradation in accuracy, seen in Table 4.4.

\subsubsection{Large Real-World Application}

The large, real-world application, used for these experiments, is JTopas [28], a Java tokenizer and parsing tool. JTopas is open-source, has an accompanying JUnit regression test suite, and is freely available from the Software-artifact Infrastructure Repository [5]. 
The symbolic execution engine, still under development at the time of evaluation, could not handle some of the classes in JTopas. These classes included more complex constructs, such as synchronized blocks, exceptions, and referencing array entries in conditional statements. Thus, these classes had to be omitted during all experiments involving JTopas. However, the experiments still yield sufficient results to demonstrate the goals of this thesis.

\section{Results}

Table 4.5, p. 85, presents the coverage calculation results of JTopas, while Table 4.6, p. 86 displays the comparable results between EMMA and ATCO. As before, ATCO's coverage results are rounded to the nearest integer for the sake of comparison.

\begin{tabular}{|c|c|c|c|c|c|c|c|c|c|c|}
\hline CD & LC & RD & St & CS & Cl & Me & Br & Ln & Lo & TR \\
\hline 0 & 4 & Yes & 2060 & 269694 & $54.17 \%$ & $49.73 \%$ & $36.21 \%$ & $44.17 \%$ & $0 \%$ & $99.99 \%$ \\
\hline 0 & 4 & No & 2060 & 26 & $54.17 \%$ & $49.73 \%$ & $36.21 \%$ & $44.17 \%$ & N/A & N/A \\
\hline 0 & 8 & Yes & 13340 & 269694 & $54.17 \%$ & $49.73 \%$ & $36.21 \%$ & $44.17 \%$ & $0 \%$ & $99.99 \%$ \\
\hline 0 & 8 & No & 13340 & 26 & $54.17 \%$ & $49.73 \%$ & $36.21 \%$ & $44.17 \%$ & N/A & N/A \\
\hline 1 & 4 & Yes & 4117 & 269694 & $54.17 \%$ & $43.78 \%$ & $31.41 \%$ & $41.3 \%$ & $0 \%$ & $99.99 \%$ \\
\hline 1 & 4 & No & 4117 & 26 & $54.17 \%$ & $43.78 \%$ & $31.41 \%$ & $41.3 \%$ & N/A & N/A \\
\hline 1 & 8 & Yes & 121399 & 269694 & $54.17 \%$ & $43.78 \%$ & $31.41 \%$ & $41.3 \%$ & $0 \%$ & $99.99 \%$ \\
\hline 1 & 8 & No & 121399 & 26 & $54.17 \%$ & $43.78 \%$ & $31.41 \%$ & $41.3 \%$ & N/A & N/A \\
\hline 2 & 4 & Yes & 4117 & 269694 & $54.17 \%$ & $43.78 \%$ & $31.41 \%$ & $41.3 \%$ & $0 \%$ & $99.99 \%$ \\
\hline 2 & 4 & No & 4117 & 26 & $54.17 \%$ & $43.78 \%$ & $31.41 \%$ & $41.3 \%$ & N/A & N/A \\
\hline 2 & 8 & Yes & 121399 & 269694 & $54.17 \%$ & $43.78 \%$ & $31.41 \%$ & $41.3 \%$ & $0 \%$ & $99.99 \%$ \\
\hline 2 & 8 & No & 121399 & 26 & $54.17 \%$ & $43.78 \%$ & $31.41 \%$ & $41.3 \%$ & N/A & N/A \\
\hline
\end{tabular}

Table 4.5: The correctness evaluation of ATCO through JTopas, a large, real-world application. The columns contain the configured call depth (CD), the configured loop count (LC), whether redundancy detection was used (RD), the number of explored symbolic states (St), the number of constraint solving operations performed during the information gathering phase and information analysis phase (CS), and the following coverage statistics: Class coverage (Cl), Method coverage (Me), Branch coverage (Br), Line coverage (Ln), and Loop coverage (Lo). The last column contains the test redundancy statistics (TR), indicating the percentage of states, hit during testing, that were already hit, i.e., redundant.

\section{Evaluation}

None of the behaviour, observed in the experiments presented in Table 4.5, have not already been observed in previous experiments. There is a reduction in branch coverage, and line 


\begin{tabular}{|c|c|c|c|c|c|c|}
\hline & \multicolumn{2}{|c|}{ Class } & \multicolumn{2}{c|}{ Method } & \multicolumn{2}{c|}{ Line } \\
\hline Tool & Total & Cov & Total & Cov & Total & Cov \\
\hline ATCO & 21 & $54 \%$ & 183 & $43 \%$ & 520 & $41 \%$ \\
\hline$E M M A$ & 21 & $95 \%$ & 183 & $72 \%$ & 520 & $74 \%$ \\
\hline
\end{tabular}

Table 4.6: The correctness evaluation of $A T C O$, by comparing its results, when measuring the code coverage of JTopas, with that of EMMA. The columns contain the tool used (Tool), and the total number (Total) of classes, methods, and executable lines found in the applications, as well as the corresponding percentage of this number to the total number of classes, methods, and executable lines that are covered (Cov).

coverage, when interprocedural analysis $(C D>0)$ is used. Also, there is a consistent $0 \%$ loop coverage, across all experiments. As mentioned in previous experiments, the presence of non-primitive conditions for branches, and symbolic loops, causes a degradation in the accuracy of coverage calculation. However, the benefits of disabling redundancy detection in these experiments are remarkable, requiring only 26 constraint solving operations to yield the same coverage results. This demonstrates the repetitive design of the JUnit test suite of JTopas. This repetitive design is further illuminated by the high test redundancy levels, detected during coverage calculation.

The comparable results between $A T C O$ and EMMA, again indicate the limitations of the current implementation of the symbolic execution engine, still under development at the time of evaluation. Since constraint solving cannot be used to determine which symbolic states are hit during testing when non-primitive types are used, none of the symbolic states are marked as hit, even though the test did, in fact, execute that specific line.

\subsection{Performance of Coverage Calculation}

The second set of experiments aims to measure the performance gains from the concurrent design of coverage calculation in ATCO. These experiments are performed with the custom application Loops, and the large, real-world application JTopas.

Each experiment is performed with varying concurrent configurations. Each experiment is executed five times, and the results are calculated as an average over the five executions. This is to account for varying loads on the experimentation environment. The average provides a more accurate approximation of the actual performance. 


\subsubsection{Custom Applications}

To increase the visibility of the performance gains of ATCO's concurrent design, the Loops application is analysed with a loop count configuration of 30. A high loop count increases the number of explored symbolic states, as well as the number of constraint solving operations during coverage calculation, as is shown in Table 4.1, p. 79.

\section{Results}

The results in Table 4.7, p. 87, displays the monitored execution times of the full information analysis phase, as well as the execution time of the longest running JUnit test case. The purpose of displaying the longest running test case is to provide a more visible indication of the benefits of $A T C O$ 's concurrent design.

\begin{tabular}{|c|c|c|c|c|c|c|}
\hline St & CS & Tst & Thr & Time & Long & Gain \\
\hline 15848 & 12549 & 3 & 1 & $40.08 \mathrm{~s}$ & $23.51 \mathrm{~s}$ & $0 \%$ \\
\hline 15848 & 12549 & 3 & 2 & $30.72 \mathrm{~s}$ & $23.97 \mathrm{~s}$ & $23.35 \%$ \\
\hline 15848 & 12549 & 3 & 3 & $27.07 \mathrm{~s}$ & $24.24 \mathrm{~s}$ & $32.46 \%$ \\
\hline
\end{tabular}

Table 4.7: The performance evaluation of $A T C O$ with the Loops custom application, configured with a loop count of 30 . The columns contain the number of explored symbolic states (St), the number of constraint solving operations performed during the information gathering phase and information analysis phase (CS), the number of JUnit tests executed (Tst), the number of configured concurrent threads (Thr), the total execution time of the information analysis phase (Time), the execution time of the longest running test (Long), and the performance gain (Gain) received from the concurrent configuration, when compared to the execution with only one concurrent thread.

\section{Evaluation}

With only one concurrent thread, all the test cases are executed sequentially. Therefore, the total execution time is the sum of the execution time of each test, together with post-processing performed by $A T C O$, such as updating coverage counters, and so on. A noticeable improvement on execution time is observed with the increase in concurrent threads. However, this experiment is small, so a large, real-world application will yield more accurate and reliable results. 


\subsubsection{Large Real-World Applications}

JTopas should provide a more reliable and accurate indication of the performance gains of $A T C O$ 's concurrent design, as it contains many classes and test cases. For these experiments, the default analysis configurations are used.

\section{Results}

As with the previous performance evaluation, the results in Table 4.8, p. 88, displays the monitored execution times of the full information analysis phase, as well as the execution time of the longest running JUnit test case.

\begin{tabular}{|c|c|c|c|c|c|c|}
\hline St & CS & Tst & Thr & Time & Long & Gain \\
\hline 4117 & 269694 & 17 & 1 & $4930 \mathrm{~s}$ & $2124 \mathrm{~s}$ & $0 \%$ \\
\hline 4117 & 269694 & 17 & 2 & $2599 \mathrm{~s}$ & $2058 \mathrm{~s}$ & $47.28 \%$ \\
\hline 4117 & 269694 & 17 & 3 & $2243 \mathrm{~s}$ & $2051 \mathrm{~s}$ & $54.50 \%$ \\
\hline 4117 & 269694 & 17 & 4 & $2245 \mathrm{~s}$ & $2101 \mathrm{~s}$ & $54.46 \%$ \\
\hline 4117 & 269694 & 17 & 5 & $2252 \mathrm{~s}$ & $2158 \mathrm{~s}$ & $54.32 \%$ \\
\hline
\end{tabular}

Table 4.8: The performance evaluation of ATCO with the JTopas application, with the default configurations for call depth (1), loop count (4), and whether redundancy detection is used (yes). The columns contain the number of explored symbolic states $(\mathbf{S t})$, the number of constraint solving operations performed during the information gathering phase and information analysis phase (CS), the number of JUnit tests executed (Tst), the number of configured concurrent threads (Thr), the total execution time of the information analysis phase (Time), the execution time of the longest running test (Long), and the performance gain (Gain) received from the concurrent configuration, when compared to the execution with only one concurrent thread.

\section{Evaluation}

The concurrent design of $A T C O$ yields notable improvements to the performance of the tool. However, the results in Table 4.8 also reveals a bottleneck in the current design. The maximum performance gain, through the current concurrent design, is determined by the execution time of the longest running test case. This is because ATCO is designed to execute each test in its own concurrent thread. So, if one thread performs, e.g., $90 \%$ of all the work, the greatest performance gain would be e.g., 10\%. While having 10 threads, each doing $10 \%$ of the work, the greatest performance gain could be closer to $90 \%$.

There is a way to alleviate this issue. All the threads, used to execute the JUnit tests, 
are stored in a thread pool. At the moment, all threads are only used to execute the JUnit tests. To avoid the bottleneck, the management of the thread pool needs to be changed. A possible option is to have two types of tasks to be assigned to threads. The first and highest priority task, is the execution of the JUnit tests. Whenever constraint solving is required for coverage calculation, $A T C O$ should first check whether any threads are idle, with idle threads indicating that no new tests need to be executed. If no idle threads are present, perform constraint solving with the current, sequential process. If idle threads are present, create a thread task for every required constraint solving operation. These operations will then execute in parallel. This solution utilises the concurrent execution of the JUnit tests, and benefits from idle threads when there are tests that require large numbers of constraint solving operations. Unfortunately, due to time constraints, this solution was never implemented in ATCO.

\subsection{Effectiveness of Generated Test Cases}

The third set of experiments aims to indicate the effectiveness of the automatically generated test cases when $A T C O$ uses the $S E T$ with the coverage results, to generate test cases for uncovered areas of an application. However, the test generation engine, still under development at the time of evaluation, imposed various limitations. These limitations caused that only the SimpleComparisons application can be used for this evaluation.

\section{Results}

Table 4.9, p. 90, presents the effectiveness of the test generation from the coverage results. The experiment was executed two times. The purpose of the first execution is to measure code coverage of the initial test suite, and automatically generate JUnit test cases. The second execution uses both the original test suite, together with the newly generated test cases, to measure the code coverage of the resulting test suite.

\section{Evaluation}

Although this experiment is small and basic, it does give an indication of the effectiveness of using symbolic execution, with coverage calculation, to automatically generate test cases. The initial JUnit test suite tests every method, but poorly tests the various branches in those 


\begin{tabular}{|c|c|c|c|c|c|c|}
\hline \multicolumn{7}{|c|}{ Before Test Generation } \\
\hline Gen & Cl & Me & Br & Ln & RH & TR \\
\hline 3 & $100 \%$ & $83.33 \%$ & $62.5 \%$ & $76.19 \%$ & 6 & $54.55 \%$ \\
\hline \hline \multicolumn{7}{|c|}{ After Test Generation } \\
\hline Gen & $\mathbf{C l}$ & Me & Br & Ln & RH & TR \\
\hline 0 & $100 \%$ & $83.33 \%$ & $100 \%$ & $90.48 \%$ & 8 & $50 \%$ \\
\hline
\end{tabular}

Table 4.9: The evaluation the automatically generated test cases through ATCO, by performing coverage calculation, and automatically generating test cases for uncovered areas. The table headers indicate the phase of the experiments, the one phase being before test generation the other being after test generation. The first column contains the number of generated test cases (Gen). Then, the columns contain the coverage statistics, which include Class coverage $(\mathbf{C l})$, Method coverage (Me), Branch coverage $(\mathbf{B r})$, and Line coverage (Ln). The next column indicates the number of symbolic states that are redundantly hit during testing $(\mathbf{R H})$, while the last column contains the test redundancy statistics (TR), as before, indicating the percentage of states, hit during testing, that were already hit, i.e., redundant.

methods. The coverage calculation results indicate these uncovered branches in the $S E T$, and tests are generated for each uncovered branch. The resulting test suite fully covers all the branches of the application.

There are still a number of uncovered lines in the application, however these are in the main method, which has a String array input parameter, that is not supported by the current test generation engine.

Notably, a reduction in test redundancy is detected, while an increase in the number of redundantly hit symbolic states is observed. This indicates that the resulting test suite does generate some redundant tests, but an overall net gain is observed. 


\section{Chapter 5}

\section{Conclusion}

This thesis investigated the practical applicability of using the static analysis formal method known as symbolic execution, to calculate code coverage of an existing JUnit regression test suite. The goal of this investigation was to determine whether this information could be used to generate thorough and efficient regression test suites, by automatically generating JUnit test cases for areas of a program currently not covered by its regression test suite. To achieve this goal, a tool named $A T C O$ was implemented. $A T C O$ is an acronym compiled from Automated Test Coverage Calculation and GeneratiOn.

$A T C O$ has shown that coverage calculation could be performed with symbolic execution. The symbolic execution information, stored in a Symbolic Execution Tree (SET), represented each method as a symbolic model of all its execution paths. With the SET, code coverage of various common testing requirements, such as class coverage, method coverage, branch coverage, and line coverage, could be measured accurately for methods with primitive data types and branching conditions. The use of the $S E T$, together with execution tracing, used to measure the coverage, also allowed for an uncommon testing requirement, i.e., loop coverage, to be measured accurately. However, coverage cannot currently be calculated for non-primitive data types, such as Objects and arrays, because the current symbolic execution engine does not support non-primitive data types. When $A T C O$, while executing a test, reaches a branch with a condition that includes non-primitive data types, and the branch is represented by more than one symbolic state, the current symbolic execution engine cannot be used to identify which of these symbolic states was actually hit. In such cases, none of the states are marked as hit, even though the branch was actually hit. 
One of the objectives was to use concurrency to reduce the time it takes to execute the coverage calculation phase. This is because constraint solving is an expensive computation. Constraint solving is used to determine which symbolic state is hit during testing, by solving the constraints on the path condition of the symbolic state, with the input parameters of the method, received during testing. If the input parameter values satisfy the path conditions of a symbolic state, that state may be marked as hit. ATCO was designed to perform coverage calculation concurrently, by executing each JUnit test case in its own concurrent thread. The results have shown significant performance gains with this concurrent design, indicating up to a $54 \%$ reduction in execution time. However, a bottleneck could be observed with this design, as the maximum performance gain is restricted by the execution time of the longest running test case. A solution to the bottleneck was proposed, but never implemented in this thesis.

Together with the concurrent design, further attempts to optimise the execution of coverage calculation included reducing the number of constraint solving operations, required to measure coverage. The first step was to only perform constraint solving if a branch has more than one symbolic state representing it, as in the case of nested or sequential if statements in a method. The second step was to ignore a symbolic state if it was previously marked as covered. Therefore, constraint solving would only be performed on a symbolic state if there was more than one symbolic state representing a branch, and if the state has not already been hit. These two steps yield significant decreases in constraint solving operations, in some cases reducing the number of operations from 269694 to 26, while still yielding identical coverage results to the case where constraint solving is always performed. However, this eliminates the ability to indicate how many times each symbolic state is hit during testing. Since this information is useful for other features, this constraint solving reduction can be activated or deactivated. Knowledge of how many times each state was hit during testing could be used to perform test redundancy detection.

Test redundancy detection was implemented to improve the efficiency of the regression test suite by identifying the level of redundancy of the current regression test suite. It is used to indicate how many of the symbolic states, hit during testing, were hit redundantly. This could be used to determine if there are tests in the regression test suite that do not test any new areas of the program. Identifying these test cases, and removing them from the suite, improves the efficiency of a test suite. 
One of the main aims of this thesis was to automatically generate JUnit test cases for the areas of a program, not being tested by the current regression test suite. The current test generation engine, still under development at the time, has limited functionality, and thus, the experiments to evaluate this feature was very basic. However, the experiments indicated that the SET and the coverage information could be used to accurately identify areas that are not covered, and tests could be effectively generated for only those areas. This information ensures that all the generated tests hit at least one previously uncovered state, during testing.

To improve the usability of $A T C O$, it was integrated into Eclipse as a plug-in, utilising the graphical user interface $(G U I)$ extensions, provided by Eclipse. This showed many usability improvements, provided by Eclipse, that analysis tools can use to encourage developers to use such tools. These usability improvements include easy file and test selection, configuration menus, persistent configurations that remain after Eclipse is closed and re-opened, and list views to display coverage results. All these improvements make such a tool easier to use, configure, and display the results in an easy-to-view format.

\subsection{Future Work}

This thesis provides a number of avenues for future development. These avenues include:

- The symbolic execution engine and test generation engine, used in this thesis, currently only supports primitive data types. An important avenue of future work is to extend the symbolic execution engine, and test generation engine, to include support for nonprimitive data types.

- ATCO executes in three distinct phases. This requires all the information of each phase to be stored, which greatly increases the resource requirements of the tool. Future developments may involve finding efficient methods of reducing the resource requirements, without significantly increasing execution times, by having the three phases interact more closely.

- This thesis used run-time information collection, in the form of execution tracing to measure coverage. However, as discussed in this thesis, there are other methods available to measure coverage. These are source code instrumentation, and intermediate code 
instrumentation. Experimenting with various methods of coverage calculation may yield interesting results, with regards to execution times.

- This thesis performs its analysis from Java byte code. This reduces the complexity of the code to analyse. However, useful information such as the actual Java source code statements, are hidden from the analysis tool. This prevents ATCO from measuring other forms of coverage criteria, such as assertion coverage. An avenue of future development involves extending the current implementation of $A T C O$ to perform its analysis directly from the Java source code.

- The JUnit test cases currently generated by ATCO only attempt to reach the symbolic state. An avenue of future development would be to extend the test generation in ATCO to evaluate the values returned from the invoked methods. These returned values may then be used to generate assertion statements for use in the JUnit test cases. This will allow for fully usable regression test suites, complete with assertions for the current functionality of an application, to be generated automatically.

- The monitoring of a manual execution with $A T C O$ currently only aims to measure code coverage of that execution. However, there is sufficient information in the SET, and additional information may be collected during execution tracing, to generate JUnit test cases that mirror the behaviour, exhibited during manual execution. Possible research in future may involve studying the effectiveness of generating such tests. This may enable users unfamiliar with the JUnit test framework, and, in fact, the Java programming language, to generate JUnit test cases, by simply testing the program manually. 


\section{Bibliography}

[1] Agitar. http://www.agitar.com/. [2]

[2] B. Aktan, G. Greenwood, and M. H. Shor. Improving evolutionary algorithm performance on maximizing functional test coverage of asics using adaptation of the fitness criteria. In Proceedings of the Evolutionary Computation on 2002. CEC'02. Proceedings of the 2002 Congress - Volume 02, CEC'02, pages 1825-1829, Washington, DC, USA, 2002. IEEE Computer Society. [34]

[3] H. Amjad. Combining model checking and theorem proving. In Technical Report UCAMCL-TR-601, University of Cambridge Computer Laboratory, 2004. [13]

[4] Java Platform Debugger Architecture. http://java.sun.com/javase/6/docs/technotes/guides/jpda/architecture.html. [6, 53]

[5] Software artifact Infrastructure Repository. http://sir.unl.edu/portal/index.php. [84]

[6] Mike Clark. JUnitPrimer.

http://clarkware.com/articles/junitprimer.html. [81]

[7] Edmund M. Clarke, E. Allen Emerson, and Joseph Sifakis. Model checking: algorithmic verification and debugging. Commun. ACM, 52(11):74-84, 2009. [13]

[8] Edmund M. Clarke and Jeannette M. Wing. Formal methods: state of the art and future directions. ACM Comput. Surv., 28(4):626-643, 1996. [11, 12, 15]

[9] Clover. http://www.atlassian.com/software/clover/. [5, 39]

[10] Alberto Coen-Porisini, Giovanni Denaro, Carlo Ghezzi, and Mauro Pezzé. Using symbolic execution for verifying safety-critical systems. In ESEC/FSE-9: Proceedings of the 8th 
European software engineering conference held jointly with 9th ACM SIGSOFT international symposium on Foundations of software engineering, pages 142-151, New York, NY, USA, 2001. ACM. [24, 28]

[11] James C. Corbett, Matthew B. Dwyer, John Hatcliff, Shawn Laubach, Corina S. Păsăreanu, Robby, and Hongjun Zheng. Bandera: extracting finite-state models from Java source code. In ICSE'00: Proceedings of the 22nd international conference on Software engineering, pages 439-448, New York, NY, USA, 2000. ACM. [14]

[12] Patrick Cousot, Radhia Cousot, Jerôme Feret, Laurent Mauborgne, Antoine Min, David Monniaux, and Xavier Rival. The ASTRÉE analyzer. In Programming Languages and Systems, Proceedings of the 14th European Symposium on Programming, volume 3444 of Lecture Notes in Computer Science, pages 21-30. Springer, 2005. [17]

[13] Christoph Csallner and Yannis Smaragdakis. JCrasher: an automatic robustness tester for Java. Softw. Pract. Exper., 34(11):1025-1050, 2004. [1, 28, 29]

[14] Christoph Csallner and Yannis Smaragdakis. Check 'n' Crash: combining static checking and testing. In ICSE'05: Proceedings of the 27th international conference on Software engineering, pages 422-431, New York, NY, USA, 2005. ACM. [29]

[15] Christoph Csallner and Yannis Smaragdakis. DSD-Crasher: a hybrid analysis tool for bug finding. In ISSTA'06: Proceedings of the 2006 international symposium on Software testing and analysis, pages 245-254, New York, NY, USA, 2006. ACM. [30]

[16] Christoph Csallner, Nikolai Tillmann, and Yannis Smaragdakis. Dysy: dynamic symbolic execution for invariant inference. In ICSE'08: Proceedings of the 30th international conference on Software engineering, pages 281-290, New York, NY, USA, 2008. ACM. $[22,27,28]$

[17] EMMA. http://emma.sourceforge.net/index.html. [36, 39, 76, 77, 81]

[18] Michael D. Ernst, Jake Cockrell, William G. Griswold, and David Notkin. Dynamically discovering likely program invariants to support program evolution. In ICSE'99, pages 213-224, 1999. [30] 
[19] Cormac Flanagan, K. Rustan M. Leino, Mark Lillibridge, Greg Nelson, James B. Saxe, and Raymie Stata. Extended Static Checking for Java. In PLDI'02: Proceedings of the ACM SIGPLAN 2002 Conference on Programming Language Design and Implementation, pages 234-245, New York, NY, USA, 2002. ACM. [17, 29]

[20] D. Geist, M. Farkas, A. Landver, Y. Lichtenstein, S. Ur, and Y. Wolfsthal. Coveragedirected test generation using symbolic techniques, 1996. [34]

[21] Raanan Grinwald, Eran Harel, Michael Orgad, Shmuel Ur, and Avi Ziv. User defined coverage - a tool supported methodology for design verification. In Proceedings of the 35th annual Design Automation Conference, DAC'98, pages 158-163, New York, NY, USA, 1998. ACM. [34]

[22] Gerard J. Holzmann and Rajeev Joshi. Model-driven software verification. In In Proc. 2001 ACM SIGPLANSIGSOFT Workshop on Program Analysis for Software Tools and Engineering (PASTE01, pages 80-89. ACM Press, 2004. [14]

[23] David Hovemeyer and William Pugh. Finding bugs is easy. In OOPSLA'04: Companion to the 19th annual ACM SIGPLAN conference on Object-oriented Programming Systems, Languages, and Applications, pages 132-136, New York, NY, USA, 2004. ACM. [17]

[24] Dan Hyde. Latitude.

http://www.eg.bucknell.edu/ cs475/f04-s05/junitsample.html. [81]

[25] Petri Ihantola. Test data generation for programming exercises with symbolic execution in java pathfinder. In Baltic Sea'06: Proceedings of the 6th Baltic Sea Conference on Computing education research, pages 87-94, New York, NY, USA, 2006. ACM. [22]

[26] JCover. http://www.codework.com/jcover/product.html. [39]

[27] JTest. http://www.parasoft.com/jsp/products/jtest.jsp/. [2, 76]

[28] JTopas. http://jtopas.sourceforge.net/jtopas/index.html. [84]

[29] JUnit.org. http://www.junit.org/. [1, 82]

[30] Cem Kaner. Software negligence and testing coverage. In Software Testing, Analysis \& Review Conference (STAR), page 313, 1996. [5, 39, 51, 52] 
[31] Sarfraz Khurshid, Corina S. Păsăreanu, and Willem Visser. Generalized symbolic execution for model checking and testing. In Proceedings of the 9th international conference on Tools and algorithms for the construction and analysis of systems, TACAS'03, pages 553-568, Berlin, Heidelberg, 2003. Springer-Verlag. [20]

[32] James C. King. A new approach to program testing. In Proceedings of the international Conference on Reliable Software, pages 228-233, New York, NY, USA, 1975. ACM. [16, 18]

[33] James C. King. Symbolic execution and program testing. Commun. ACM, 19(7):385-394, 1976. $[1,2,6,18]$

[34] Flemming Nielson, Hanne R. Nielson, and Chris Hankin. Principles of Program Analysis. Springer-Verlag New York, Inc., Secaucus, NJ, USA, 1999. [15, 16]

[35] Martin Ouimet. Formal software verification: Model checking and theorem proving. In Embedded Systems Laboratory Technical Report, Massachusetts Institute of Technology, 2005. $[13,14]$

[36] Tuomo Pyhälä and Keijo Heljanko. Specification coverage aided test selection. In Johan Lilius, Felice Balarin, and Ricardo J. Machado, editors, Proceeding of the 3rd International Conference on Application of Concurrency to System Design (ACSD'2003), pages 187-195, Guimaraes, Portugal, June 2003. IEEE Computer Society. [34]

[37] Stephen F. Siegel, Anastasia Mironova, George S. Avrunin, and Lori A. Clarke. Using model checking with symbolic execution to verify parallel numerical programs. In ISSTA'06: Proceedings of the 2006 International Symposium on Software Testing and Analysis, pages 157-168, New York, NY, USA, 2006. ACM. [27]

[38] CHOCO Team. choco: an Open Source Java Constraint Programming Library. In Ecole des Mines de Nantes. http://www.emn.fr/z-info/choco-solver/pdf/choco-presentation.pdf, 2010. [50]

[39] Aaron Tomb, Guillaume Brat, and Willem Visser. Variably interprocedural program analysis for runtime error detection. In ISSTA'07: Proceedings of the 2007 International Symposium on Software Testing and Analysis, pages 97-107, New York, NY, USA, 2007. ACM. [6, 26, 27] 
[40] Shmuel Ur and Avi Ziv. Off-the-shelf vs. custom made coverage models, which is the one for you? [34]

[41] Concurrency Utilities.

http://download.oracle.com/javase/6/docs/technotes/guides/concurrency/overview.html. [41]

[42] Raja Vallée-Rai, Phong Co, Etienne Gagnon, Laurie Hendren, Patrick Lam, and Vijay Sundaresan. Soot - a java bytecode optimization framework, 1999. [42, 43]

[43] Willem Visser, Klaus Havelund, Guillaume Brat, and SeungJoon Park. Model checking programs. In ASE'00: Proceedings of the 15th IEEE international conference on Automated Software Engineering, page 3, Washington, DC, USA, 2000. IEEE Computer Society. [14]

[44] T. Xie, D. Marinov, W. Schulte, and D. Notkin. Symstra: A framework for generating object-oriented unit tests using symbolic execution. In 11th International Conference on Tools and Algorithms for the Construction and Analysis of Systems, pages 365-381. Springer Verlag, April 2005. [76]

[45] Tao Xie. Improving effectiveness of automated software testing in the absence of specifications. PhD thesis, University of Washington, Seattle, WA, USA, 2005. AAI3183442. $[1,2,6,63]$

[46] Qian Yang, J. Jenny Li, and David M. Weiss. A survey of coverage-based testing tools. Comput. J., 52:589-597, August 2009. [2, 5, 7, 39]

[47] Hong Zhu, Patrick A. V. Hall, and John H. R. May. Software unit test coverage and adequacy. ACM Comput. Surv., 29(4):366-427, 1997. [2, 4, 30, 31, 32, 34, 35, 36, 37, 38, 39, 52] 